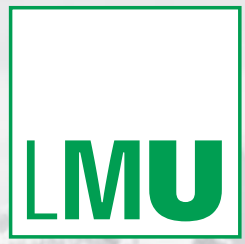

LUDWIG-

MAXIMILIANS-

UNIVERSITÄT

MÜNCHEN

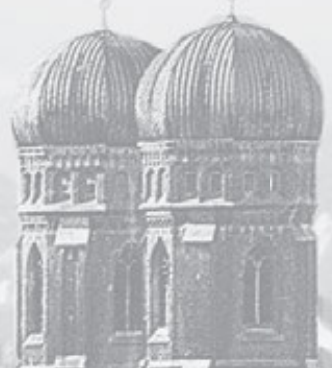

$20^{\text {th }}$ International Conference on

Multimedia in Physics Teaching and Learning
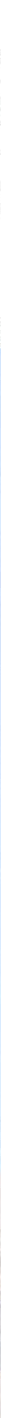


\begin{tabular}{|c|c|c|c|}
\hline & Wed, 9 Sep & Thu, 10 Sep & Fri, 11 Sep \\
\hline 09:00 - 10:00 & Opening Ceremony & $\begin{array}{c}\text { Plenary Lecture } \\
\text { Michael Dubson }\end{array}$ & $\begin{array}{c}\text { Plenary Lecture } \\
\text { Wouter van Joolingen }\end{array}$ \\
\hline 10:00 - 11:00 & $\begin{array}{l}\text { Plenary Lecture } \\
\text { Jochen Schieck }\end{array}$ & $\begin{array}{c}\text { Plenary Lecture } \\
\text { Christian Hackenberger }\end{array}$ & $\begin{array}{c}\text { Plenary Lecture } \\
\text { David Lowe }\end{array}$ \\
\hline 11:00 - 11:30 & Coffee Break & Coffee Break & Coffee Break \\
\hline $11: 30-13: 00$ & $\begin{array}{l}\text { 1A Invited Symposium REP } \\
\text { 1B Invited Symposium IWB }\end{array}$ & $\begin{array}{c}\text { 3A Parallel Session QP } \\
\text { 3B Parallel Session VRL/MAP } \\
\text { 3C Workshop CAM } \\
\end{array}$ & $\begin{array}{c}\text { 6A Parallel Session SIM/VID } \\
\text { 6B Invited Symposium ASS } \\
\text { 6C Workshop MAP } \\
\end{array}$ \\
\hline $13: 00-14: 00$ & Lunch & Lunch & Closing Ceremony \\
\hline $14: 00-16: 00$ & $\begin{array}{l}\text { 2A Invited Symposium VRL } \\
\text { 2B Invited Symposium ILA }\end{array}$ & $\begin{array}{l}\text { 4A Invited Symposium iMP } \\
\text { 4B Invited Symposum GBL } \\
\text { 4C Parallel Session MM }\end{array}$ & Lunch \\
\hline $16: 00-16: 30$ & Coffee Break & Coffee Break & \\
\hline $16: 30-18: 30$ & $\begin{array}{l}\text { Poster Session } \\
\& \\
\text { Welcome Party }\end{array}$ & $\begin{array}{c}\text { 5A Invited Symposium QP } \\
\text { 5B Parallel Session ILA } \\
\text { 5C Workshop VRL }\end{array}$ & \\
\hline $18: 30-20: 30$ & Guided Tour & Conference Dinner & \\
\hline $20: 30-23: 00$ & & & \\
\hline
\end{tabular}




\section{Program and Book of Abstracts}





\title{
$20^{\text {th }}$ International Conference on
}

\author{
Multimedia in Physics Teaching and Learning
}

\section{Program and Book of Abstracts}

\author{
MPTL 2015 International Conference
}

September 9-11, 2015

at LMU Munich, Germany

Organized by:

Multimedia in Physics Teaching and Learning (MPTL)

Chair of Physics Education, Faculty of Physics, LMU Munich

With the support of:

German Research Foundation (DFG)

European Physical Society (EPS) - Physics Education Division

German Physical Society (DPG)

Wilhelm und Else Heraeus-Stiftung

Ludwig-Maximilians-Universität München (LMU)

The city of Munich 


\title{
COMMITEES
}

\author{
Scientific Advisory Board \\ Wolfgang Christian, Davidson College, USA \\ Ton Ellermeijer, CMA, Amsterdam, Netherlands \\ Francisco Esquembre, University of Murcia, Spain \\ Raimund Girwidz, LMU Munich, Germany \\ Tomasz Greczyło, University of Wrocław, Poland \\ Bruce Mason, University of Oklahoma, USA \\ Marisa Michelini, University of Udine, Italy
}

\section{Program Commitee}

Nick Braithwaite, The Open University, UK

André Bresges, University of Cologne, Germany

Wolfgang Christian, Davidson College, USA

Sebastián Dormido, UNED, Spain

Ton Ellermeijer, CMA, Netherlands

Francisco Esquembre, Universidad de Murcia, Spain

Raimund Girwidz, LMU Munich, Germany

Tomasz Greczylo, University of Wroclaw, Poland

Jochen Kuhn, University of Kaiserslautern, Germany

Bruce Mason, University of Oklahoma, USA

Marisa Michelini, University of Udine, Italy

Dean Zollman, Kansas State University, USA

\section{Local Organizing Commitee}

Raimund Girwidz, LMU Munich, Germany

Stefan Richtberg, LMU Munich, Germany

Lars-Jochen Thoms, LMU Munich, Germany

Bianca Watzka, LMU Munich, Germany

\section{MPTL 2015 Program and Book of Abstracts}

(C) 2015 Ludwig-Maximilians-Universität München, Chair of Physics Education

Back cover image: Rudolf Sterflinger, München Tourismus, Nr. 0711-2

Volume editor: Lars-Jochen Thoms, Raimund Girwidz

Volume number: 39 B

Published by the European Physical Society

Managing editor: P. Helfenstein, Mulhouse

Printed in Münster by Verlagshaus Monsenstein \& Vannerdat in cooperation with Ludwig-Maximilians-Universität München, University Library

The electronic version of this publication is available via

http://nbn-resolving.de/urn:nbn:de:bvb:19-epub-25097-8 


\section{TABLE OF CONTENTS}

Plenary Talks

Invited Symposium REP:

Visual Representations in Physics Education

Invited Symposium IWB:

Perspectives on Interactive Whiteboard Use in Physics Instruction

Invited Symposium VRL:

Virtual and Remote Labs in Practice...

Invited Symposium ILA:

Concepts to Initialize Learning Activities With Modern Media ...

Parallel Session QP:

Multimedia in Teaching and Learning Quantum Physics. ...

Parallel Session VRL/MAP:

Virtual and Remote Labs in Practice / Concept Mapping. . .

Workshop CAM:

The Amazing World of Ionizing Particles: Experiments With MX-10 Particle Camera

Invited Symposium iMP:

iMobilePhysics (iMP): Using Smartphone, Tablet-PC \& Co. as Experimental Tools . .

Invited Symposium GBL:

Game-Based Learning in Physics and Physics Education

Parallel Session MM:

Multimedia in Physics Teaching and Learning.

Invited Symposium QP:

Multimedia in Teaching and Learning Quantum Physics...

Parallel Session ILA:

Concepts to Initialize Learning Activities With Modern Media ...

Workshop VRL:

Remote Controlled Experiments for High School and Undergraduate Physics Students....

Parallel Session SIM/VID:

Simulations and Videos in Physics Teaching and Learning ....

Invited Symposium ASS:

Incorporating Multimedia and ICT in Physics Education:

Focus on Learning Paths and Assessment. .

Workshop MAP:

Creating Interactive, Online Concept Maps using Free Software.

Poster Abstracts 


\section{Welcome to Munich}

Dear participants of the 20th MPTL Conference,

Dear Sir or Madam,

I may warmly welcome you in Munich. To say it right away: You could not have chosen a better place for your conference. Munich is a city of knowledge and science.

We have an impressive academic landscape and numerous internationally recognized research institutes. They are the beating heart of the science city of Munich. All the more, I am pleased that you contribute with your conference to this pulse.

Physics is a fascinating basic science. Why are things as they are? What is matter, what energy, what are their interactions in space and time? What are the laws of nature? To give people the knowledge about it is a challenging and exciting task. Like Isaac Newton said, "I don't know what I may seem to the world, but, as to myself, I seem to have been only like a boy playing on the sea shore, and diverting myself in now and then finding a smoother pebble or a prettier shell than ordinary, whilst the great ocean of truth lay all undiscovered before me." Against this background, your work is like maybe even a mission. To serve this mission with multimedia resources, was certainly a quantum leap forward in knowledge transfer.

Learning, Teaching, Multimedia - there come together already taken alone complex matters. And the linking of these matters facilitates interdisciplinary exchange. As part of your MPTL conference you will definitely share experiences and results, discuss new approaches, might try out new techniques, but certainly develop new ideas.

„Pleasure in the job puts perfection in the work.”, said Aristotle. I wish that Munich, due to its special flair, its remarkable architecture, embedded in the gravel plain of the stunning pre-alpine landscape with its mountains and lakes, will contribute to this pleasure in the job. That you get a lot of inspiration to generate these certain ideas that enable an exciting physics class.

On a good success of your convention!

Best regards,

Major Josef Schmid

Head of Department of Labor and Economic Development 


\section{Welcome to LMU Munich}

Dear Colleagues,

It is a great pleasure for us to welcome you in Munich for the 20th MPTL conference.

We come together again for our annual conference to present new ideas, to learn about new interesting concepts, and to discuss computer-based learning in physics. Also for those who are new, we wish you to find collaborations with researchers, teachers and instructors who are involved in teaching with multimedia. A warm welcome also to all participating GIREP members. By participating in the conference you revive our close collaboration with the International Research Group on Physics Teaching (GIREP). We are proud to host the MPTL conference, and hope that our preparations will lead to productive work as well as to enjoyable days in Munich.

The annual MPTL meeting gives us opportunities to exchange results of our work, which is important to improve physics education on school and university level. Thereby, we will focus mainly on three parts of scientific activities: First, MPTL conferences reach a high level on presenting new hard and software technologies for teaching and learning. New concepts, best practice examples and innovative ideas can be discussed. Second, we also have dedicated empirical studies about important factors for effective learning with modern media. Third, assessment of and with digital media is a topical issue.

The number of accepted presentations is high, compared to former meetings. This offers us the opportunity to cover a broader field and special issues. However, we will take care, not to lose the options to discuss things in expert groups with manageable numbers. Thematic sessions and invited symposia will bring together experts with special interests and help us to keep an intimate atmosphere in specific groups. Furthermore, to assist this intention, also the coffee breaks, the lunches, the conference dinner and a sight-seeing-tour offer many occasions for informal discussions, networking, renewing old ties, making new friends and to inspire and to create new projects that will help us to improve the quality of physics education. We hope that the conference will be a socially enjoyable experience for all participants.

Organizing the conference would not have been possible without the help of many people and organizations. We would like to acknowledge the support of the German Research Foundation (DFG), the European Physical Society (EPS), the German Physical Society (DPG) and the Wilhelm und Else Heraeus-Stiftung, the Ludwig-Maximilians-Universität (LMU), the Faculty of Physics at the Ludwig-Maximilians-Universität, the city of Munich, as well as our colleagues at the Ludwig-Maximilians-Universität. Thanks also to the Congress Consultancy of the LMU and the Convention Bureau Munich for their support.

We are also deeply indebted to the many reviewers and the Chairpersons of the conference sessions without whose contribution it would not have been possible to put together this scientific program.

On behalf of the Organizing Committee, we wish you fruitful, productive and happy days at the LMU.

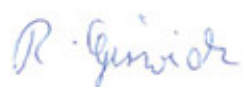

Raimund Girwidz

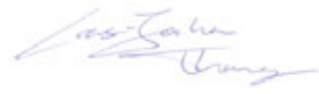

Lars-Jochen Thoms 


\section{Your Way to the LMU Main Building}

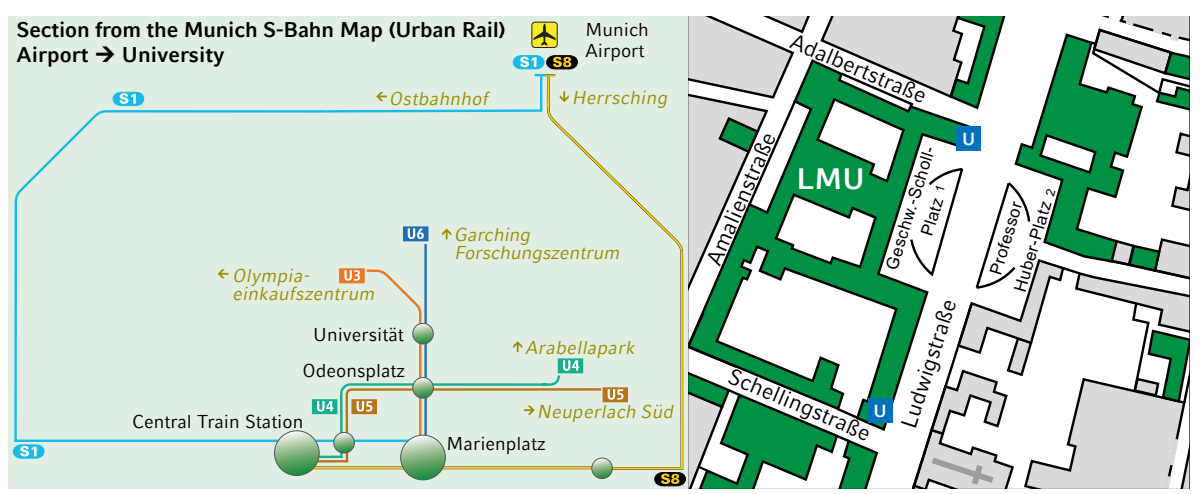

Address:

Ludwig-Maximilians-Universität München

Geschwister-Scholl-Platz 1

80539 München

Subway station Universität on lines U3 and U6.

\section{Your Way to the Reception Desk (R) and the Seminar Rooms (A, B \& C)}

1. Face the LMU main building (Hauptgebäude).

2. Take the first door on the right in front of the main entrance.

3. Go upstairs to the intermediate floor (Zwischengeschoss).

4. Find the reception desk on the left.

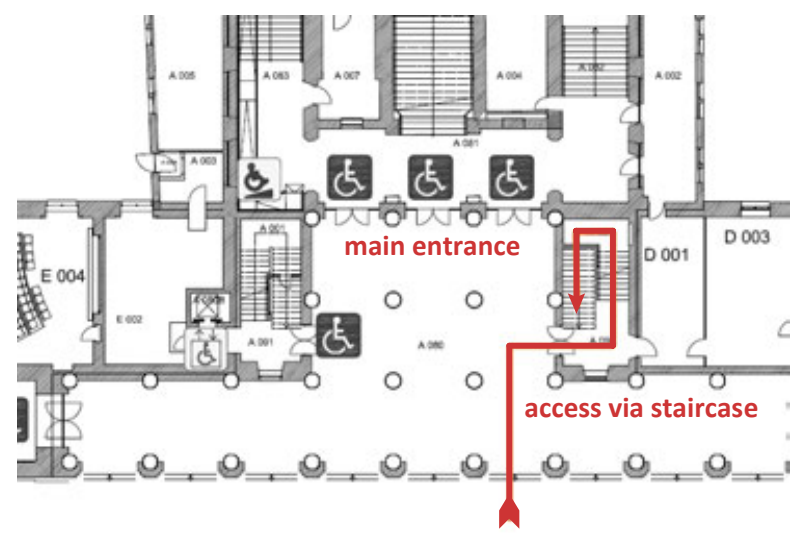

Geschwister-Scholl-Platz 1

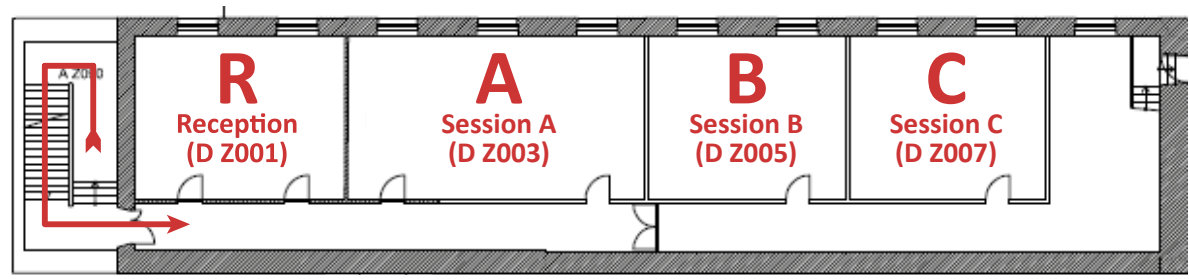




\section{Your Way to the Lecture Hall (H)}

Plenary lectures will be held in the lecture hall D 209 in the second floor above the reception desk and the seminar rooms.

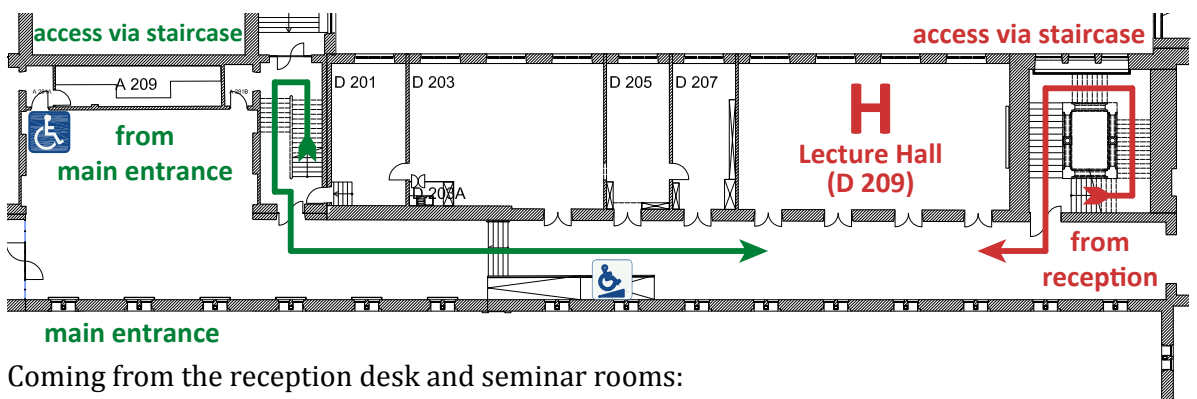

1. Go upstairs to the second floor.

2. Leave the staircase, turn right.

3. Find the lecture hall on the right.

Coming from the main entrance:

1. Face the LMU main building (Hauptgebäude).

2. Take the first door on the right in front of the main entrance.

3. Go upstairs to the second floor, leave the staircase, turn left, go straight forward.

4. Find the lecture hall on the left.

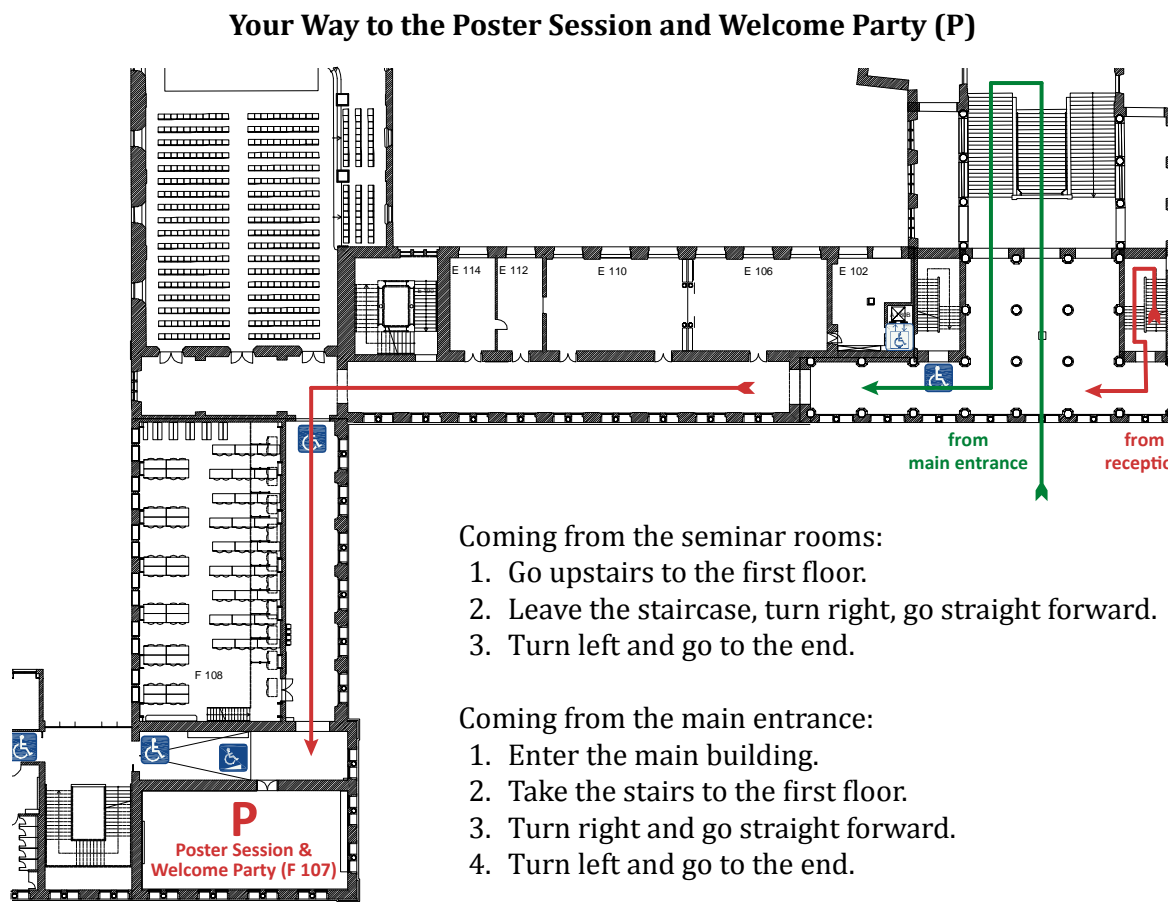




\title{
PLENARY TALKS
}

\section{Particle Physics - Three Years After the Discovery of the Higgs Jochen Schieck, Austria}

\author{
Apples vs. Oranges: \\ Comparison of Student Performance in a MOOC vs. a Brick-and-Mortar Course \\ Michael Dubson, USA
}

A Dance With Electrons

Christian Hackenberger, Germany

Drawing-Based Modelling to Foster Early Science Learning Wouter van Joolingen, Netherlands

\begin{abstract}
Remote Laboratories and Mediated Interactions:
The Real Opportunity for Enhancing Learning
\end{abstract}

David Lowe, Australia 


\section{Particle Physics - Three Years After the Discovery of the Higgs \\ Jochen Schieck, Austria}

With the discovery of the Higgs-Boson in 2012 all particles of the Standard Model of Particle Physics have been discovered. All particle physics measurements are consistent with predictions, however, we know that the Standard Model can only be an effective theory. The theory does not contain gravity, the intrinsic CP-violation is not enough to describe the observed matter-antimatter asymmetry in the universe and it doesn't contain a particle candidate for Dark Matter. I will briefly review the status of the Standard Model of particle physics and it's key measurements, including the discovery of the Higgs-Boson at CERN. The open questions of modern particle physics will be presented, theoretical scenarios which could solve this problems discussed and the experimental program summarised, including latest results from the restart of the LHC in 2015. Education and outreach is an important task of the international particle physics community. A variety of tools is available to explain students the main concepts of particle physics and, even more important, give them an idea how research in this field is performed. I will show some of these tools and will present so-called hands-on exercises for students.

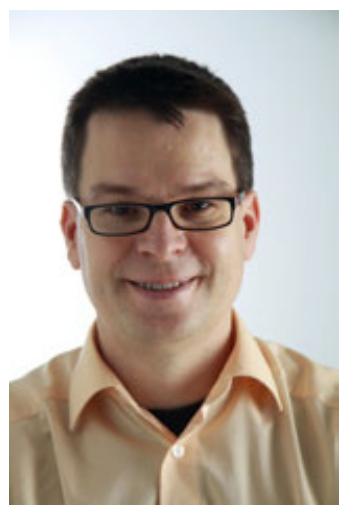

Jochen Schieck studied physics at the University of Heidelberg between 1992 and 1996. In 1999, he received his PhD from the University of Heidelberg working on the OPAL-experiment at CERN. After research stay in the USA, working at the Babar-Experiment at the Stanford Linear Accelerator Centre he moved back to Germany, working for several years at the Max Planck Institute for Physics. There he joined the ATLAS experiment, one of the two multi-purpose experiments at the LHC at CERN, which discovered the Higgs particle in 2012. In 2010, he was appointed as professor at the LMU Munich and in 2013, he moved as director to the Institute for High Energy Physics of the Austrian Academy of Sciences. He is also full professor at the Vienna University of Technology. He is a principle investigator of the cluster of excellence "Origin and Structure of Universe" and received funding from the $B M B F$ for the Belle II project. Currently he focuses on resolving the particle nature of Dark Matter, but he is also still involved at the LHC at CERN and at the Belle II experiment in Japan. 


\section{Apples vs. Oranges: \\ Comparison of Student Performance in a MOOC vs. a Brick-and-Mortar Course \\ Michael Dubson, USA}

In the fall of 2013, my colleagues and I taught the calculus-based introductory physics course to 800 tuition-paying students at the University of Colorado at Boulder. At the same time we taught a free massive open online course (MOOC) version of the same course, through Coursera.com. The initial enrollment in the MOOC was 10,000 students, of whom 255 completed the course. Students in both courses received identical lectures, homework assignments, and timed exams. We present data on participation rates and exam performance for the two groups. We find that the MOOC is like a drug targeted at a very specific population. When it works, it works well, but it works for very few students. This MOOC worked well for older, well-educated students, who already had a good understanding of Newtonian mechanics. I will include a brief history of the successes and failures of technological innovations in American public education.

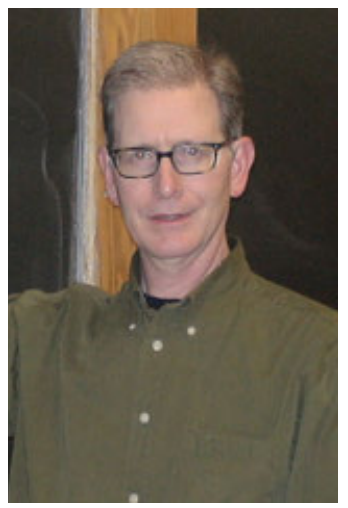

Michael Dubson is a Senior Instructor in the Physics Department at the University of Colorado at Boulder, where he is Associate Chair for Undergraduate Studies and a member of the PER group. He has a BS in Physics from the University of Illinois at Champaign-Urbana (1978) and a PhD in condensed matter experiment from Cornell University (1984). He began his career as a condensed matter researcher at Michigan State University. In 1995, he switched fields and joined the faculty at Colorado, where he works on undergraduate curriculum development and interactive instruction. He has won several teaching awards, including the 2006 American Association of Physics Teachers Education Award. He is also a software designer for PhET (http://phet.colorado.edu), a suite of free interactive simulations for science education. 


\section{A Dance With Electrons \\ Christian Hackenberger, Germany}

Although Electrons are very small particles, they are the largest thing around us. Nearly everything we experience is based on the properties of Electrons. From Chemistry to modern Computers, from Material characteristics to the spectrum of an LED lamp, you can't escape electrons. Electrons are charged particles, reacting to the electric field of light, which makes them, in principle, easy to measure. The hard part is that electrons can be very fast. For instance, in order to observe the dynamics of electronic changes in an atom you need a light pulse that is less than 100 attoseconds long, which is extremely short. Our research is centered around creating and utilising these extremely short light pulses. Here, I will present several of our innovative results and devices and discuss the ways in which we can communicate this fascinating, but nevertheless complicated, topic to the public.

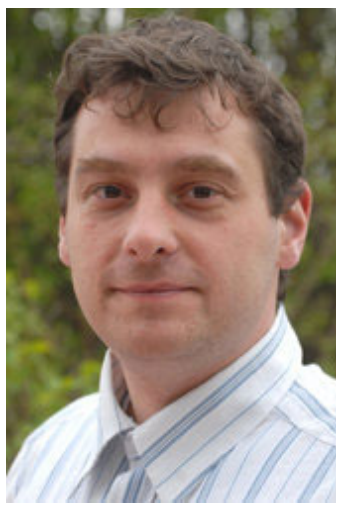

Christian Hackenberger studied undergraduate Physics at the TU in Munich, before completing his PhD in theoretical solid state Physics at the Center for Electronic Correlations and Magnetism at the University of Augsburg. During his studies he learned to use image editing, 3D imaging, and animation tools, becoming completely amazed by the possibilities. After finishing his PhD he took the chance to use both his education in physics and his imaging expertise, joining the team of Ferenc Krausz in 2008. Today, he works together with an international team of scientists on every aspect of science visualisation, whether it is designing covers, developing and producing animations to explain the details of physics to a broader audience, or preparing graphs, illustrations, and images for publications, press releases, and talks. 


\section{Drawing-Based Modelling to Foster Early Science Learning \\ Wouter van Joolingen, Netherlands}

Science is all about creating and evaluation models. Models are used to describe and understand phenomena. Models often have both a visual and a mathematical character and more and more computer simulations are used to generate predictions of the behaviour of complex systems. Because of this central role of models in science, students should learn about models and the processes of modelling. The goal of the research presented in this presentation is to make modelling accessible to young students (from age 8) by focussing on the visual aspects of modelling by creating models based on drawings. Students draw a visual representation of models, annotate them with behavior for elements in the drawing, resulting in a computer simulation that turns the drawing into an animation. I will present the drawing-based modelling method based on examples from domains such as crowd management, evolution and astronomy, as well as studies into the processes and learning outcomes in terms of domain knowledge and scientific reasoning skills.

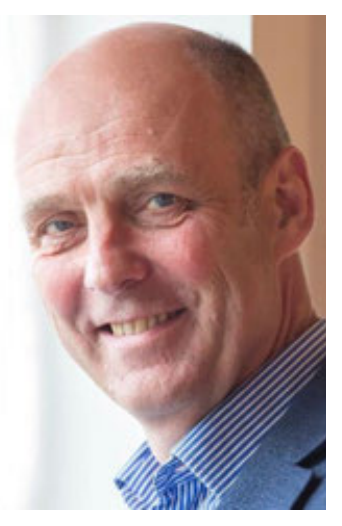

Wouter van Joolingen studied Physics at Leiden University where he graduated in 1987. In 1993 he received his Ph.D. from Eindhoven University of Technology on the use of computer simulations for inquiry learning. In 1992 he moved to the University of Twente, working on the SMISLE and Servive projects in which the SimQuest authoring system was developed. After a year in industry Wouter worked for the University of Amsterdam from 1998 to 2004, where he worked in the Co-Lab project. In 2004 he returned to Twente and was professor on the topic of "Computational Modeling in Educational Settings". As of May 1, 2015 he is scientific director of the Freudenthal Institute at the University of Utrecht. Wouter has won the "Outstanding publication award" (EARLI, 1999, together with Ton de Jong), and the European academic software award for SimQuest (EASA, 2000, with the whole SimQuest team). In 2009 he was awarded the AECT Distinguished Development Award, for his work on the development of SimQuest and Co-Lab. 


\section{Remote Laboratories and Mediated Interactions: The Real Opportunity for Enhancing Learning}

David Lowe, Australia

Remotely-accessed laboratories have grown in sophistication, and can provide significant benefits including flexibility of access and the opportunity for shared-access. However existing remote labs largely duplicate conventional labs and ignore the opportunities to reshape the ways in which we think about laboratory education. The mediated interface inherent in accessing remote labs makes it feasible to both augment and guide the interaction in ways that are impossible or impractical with traditional hands-on laboratories. Imagine a magnetics experiment where the view of the equipment is overlayed with a representation of the magnetic field. Imagine a radioactivity experiment where the student instructions are automatically adapted based on student behaviour ('what might happen if you took a longer reading?'). In this presentation the opportunities for reshaping laboratory experiences and enhancing student learning will be explored.

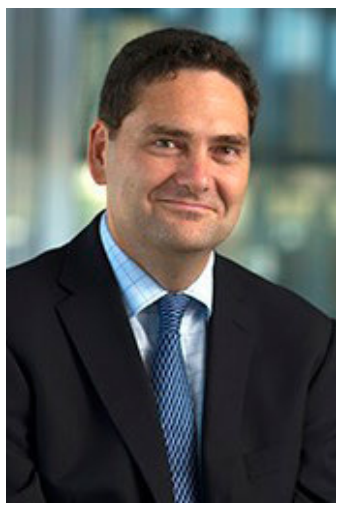

Professor David Lowe is Associate Dean (Education) and Professor of Software Engineering in the Faculty of Engineering and IT at the University of Sydney. He is also the CEO of the not-forprofit organisation The LabShare Institute and past-president of the Global Online Laboratory Consortium. He started his career in industry as a control systems engineer, but has subsequently ranged across fields as diverse as computer vision, software engineering, web development and real-time systems. He currently has active research interests in the area of realtime control of embedded systems in the web environment, and remote access to, and control of, physical laboratory systems. He has published widely, including three textbooks. David is also passionate about supporting student learning, educational innovation and promoting interest in STEM careers. 


\title{
INVITED SYMPOSIUM REP:
}

\author{
VISUAL REPRESENTATIONS IN PHYSICS EDUCATION \\ Víctor López Simó (Chairperson), Autonomous University of Barcelona, Spain \\ Raimund Girwidz (Discussant), LMU Munich, Germany
}

Visual representations have always played a central role in physics education. Images for physics teaching are depicted both in classic textbooks and in modern animations and simulations. They can provide students information that written text or equations do not allow, and it can help students to better understand physics concepts at all the educational levels. However, the idea that pictures are useful per se should not be given for granted, since previous researches have demonstrated that the optimal students' understanding of representations only occurs under some conditions: students' previous knowledge on the domain, students' visual ability, appropriate external support or adequate visual design.

In this symposium we present four recent researches focused on the role of visual representations in physics teaching and learning. These researches concern a wide variety of physics topics (mechanics and waves, astronomy, electricity and magnetism, relativity and quantum physics), and also a variety of educational levels (from primary school to university). The discussion that emerges from these four researches aims to provide new and more effective means of communicating physics concepts through visual representations.

\section{Students' Interpretation of Dynamic Representations Displayed in Simulations Víctor López Simó Autonomous University of Barcelona, Spain}

\author{
Students' Difficulties in Interpreting Images and Simulations \\ About Astronomy Phenomena \\ Italo Testa ${ }^{1}$, Silvio Leccia ${ }^{2}$ and Emanuella Puddu ${ }^{2}$ \\ ${ }^{1}$ University "Federico II" of Naples, Italy \\ ${ }^{2}$ INAF-Astronomic Observatory of Capodimonte, Naples, Italy
}

\author{
Involving Students in Visual Representations in Physics: \\ From Textbook Drawings to Digital Simulations \\ Berit Bungum \\ Norwegian University of Science and Technology, Norway
}

\author{
Why Point Particles Lead to a Dead End: \\ A New Visualization Scheme for Magnetism Based on Quantum States \\ Daniel Laumann and Stefan Heusler \\ Westfälische Wilhelms-Universität Münster, Germany
}




\section{Students' Interpretation of Dynamic Representations Displayed in Simulations Víctor López Simó \\ Autonomous University of Barcelona, Spain}

\section{Introduction and Theory}

The visual representations depicted in educational simulations provide students information that other means of instruction do not, allowing them to develop deeper and richer concepts understanding than they otherwise might (Wieman, Adams, \& Perkins, 2008). However, the idea that pictures are more communicative and "easy" to read than written text leads people to assume that when students read a visual representation they understand it correctly. Previous studies have refuted this assumption (Ametller \& Pintó, 2002; Colin, Chauvet, \& Viennot, 2002; Stylianidou, 2002; Testa, Monroy, \& Sassi, 2002), and it has been shown that an image which has not been well designed may transmit wrong ideas, and also that a lack of knowledge of the visual language may hinder the interpretation of an image. So, images cannot be considered trivially understandable and transparent.

Concept and Implementation

In this presentation we expose the investigation about how a group of secondary school students read and understand the visual representations displayed in different physics simulations available in PhET repository. These simulations concern different physics topics, such as friction, sound propagation and electromagnetic induction. In order to carry out the research, we selected a group of 20 students between 14 and 16 years old, and for each student, we carried out video-recorded individual semi-structured interviews.

\section{Objectives and Assessment}

The research allows to identify different kind of students' difficulties, that can be addressed both scaffolding students in their reading, and also introducing some changes in the visual design of simulations. A wide range of typologies of reading difficulties have been identified: related to the compositional structure, related to the relevance given to visual elements, difficulties to deal with multiple representations, and difficulties to perceive and interpret dynamic information.

Each difficulty is associated to one or more visual features. Science's specificities (such as models, scales and the nature of scientific representations) play an important role in the reading process, and thus, general statements about difficulties in reading images have to be reviewed from the point of view of content. Furthermore, since the same difficulties appear when different students read different simulations, they also can be extrapolated to other physics' simulations. Once again we realise the important role of the critic reading of images that teachers should develop when their students use scientific simulations, scaffolding the images' reading process. 


\author{
Students' Difficulties in Interpreting Images and Simulations \\ About Astronomy Phenomena \\ Italo Testa ${ }^{1}$, Silvio Leccia ${ }^{2}$ and Emanuella Puddu ${ }^{2}$ \\ ${ }^{1}$ University "Federico II" of Naples, Italy \\ ${ }^{2}$ INAF-Astronomic Observatory of Capodimonte, Naples, Italy
}

\title{
Introduction and theory
}

Teaching materials nowadays increasingly exploit images and animations in the attempt to improve students' understanding of scientific concepts, especially in physics. In this paper, we will investigate the difficulties students may encounter when interpreting images and simulations concerning astronomy topics. The main reason for this choice is that school textbooks increasingly feature Earth photographs, Sun-Moon-Earth system schematic representations, and graphs (as the H-R diagram). Moreover, planetariums and science centers increasingly offer to visitors realistic simulations using software packages as Celestia, Starry Nights, Stellarium. At the same time, astronomy is a content area where students frequently hold a variety of alternative conceptions. Some authors have pointed out that the persistence of such alternative conceptions may be due also to the difficulty in reading and interpreting the images commonly used in school textbooks. Therefore, the research question that guided the study was: do images and animations about basic astronomical phenomena support or hinder learning of the related scientific content?

We adopted for the analysis of images and animations a socio-semiotic theoretical framework inspired by the works by Kress and Van Leeuwen (1996). According to this framework, the possibility of combining together different types of representations may generate difficulties in the interpretation of the message(s) encoded within an image or simulation. Drawing on such framework, we categorized images and simulations according to their representational structures, namely the way in which symbols and signs are organized with the aim of expressing the concepts to be communicated.

\section{Concept and Implementation}

Six images from a textbook currently used in Italian secondary schools and two simulations were first analyzed according to the above framework. Then, we designed an interview protocol in which it was requested to answer one or more questions about the phenomenon represented in the images and the animations to investigate the role of the iconic features of the images and simulations. About 30 secondary students participated in the study. All students were interviewed for about 40 minutes after the participation to an eight hour out-of-school activity about the astronomy topics targeted in the images and simulations.

\section{Objectives and Assessment}

Our analysis shows that the chosen images and simulations mostly did not help students to grasp the represented concept. This result confirms the difficulties generically pointed out in previous studies. The analysis of interviewees' responses suggests also that students' difficulties are plausibly linked to specific features as the compositional structure, and the presence of real/symbolic elements and different images in the same iconic frame. Our results may be useful to improve the design of image-based instructional materials to be implemented in school practice. 


\author{
Involving Students in Visual Representations in Physics: \\ From Textbook Drawings to Digital Simulations \\ Berit Bungum \\ Norwegian University of Science and Technology, Norway
}

\begin{abstract}
Introduction and Theory
Visual representations are important not only for learning of subject matter, but also for how students conceive the subject. This contribution presents a study of images in Norwegian physics textbooks on secondary level through a century, how they have changed with regards to purpose and abstraction, and how they by visual means involve students in the field of physics. Modern ICT give rich opportunities for teaching tools that provide for more complex and interactive involvement of students in physics. Examples of visualizations used in digital teaching tools in physics are classified according to results from the textbook study, in order to see what opportunities and challenges recent technology provides with regards to involving students visually in physics.

Images are analysed by means of a framework of three dimensions: Content specialisation refers to the degree to which a specialised language of symbols and specialised settings are used. Formality refers to the degree of abstraction and use of techno-scientific codes, where realistic images and conventional images represent endpoints. The dimension of framing is built on Bernstein's work in educational philosophy. Used on visual communication, the concept refers to the degree to which the reader is invited to participate in what is taking place in the image. Weak framing means that the image invites the reader to participate, while strong framing signifies that a strong boundary is created between the image and the reader.
\end{abstract}

\title{
Research Methods and Material
}

The sample of the study consists of nine Norwegian physics textbooks for secondary school published in the period from 1943 till present, some published in numerous editions and used in schools for several decades. They are intended mainly for a level corresponding to upper secondary school, but some also for lower secondary level. Analysis of images was undertaken by characterising individual images by means of the dimensions provided described above. A sample of images used in the digital teaching resources in the project ReleQuant are compared with the findings based on the textbook analysis.

\section{Findings and Conclusion}

Ways of involving students in physics through visual representations:

It is found that while older images are dominated by realistic drawings of objects, often for use in experiments, images have an increasing level of abstraction in more recent textbooks. By use of photos, newer textbook may also have a hidden abstraction in their images, as the purpose of realistic representations may be to communicate abstract subject matter. Even if new textbooks far more often show people, for example scientists at work, the older images typically have weaker framing, meaning that they involve the reader more strongly in the physics activity in the image than what is the case with images in today's textbooks.

Digital visualizations i terms of simulations have similarities with how the older textbooks involve the students in physics activities by visual means. However, it is also found that digital visualizations may be far more complex, which combined with high content specialization might miss out on communicating well with the student. 


\author{
Why Point Particles Lead to a Dead End: \\ A New Visualization Scheme for Magnetism Based on Quantum States \\ Daniel Laumann and Stefan Heusler \\ Westfälische Wilhelms-Universität Münster, Germany
}

\title{
Introduction and Theory
}

Magnetism constitutes a fundamental topic of physics education from elementary school up to university level. On one side, vivid experiments and useful phenomenological attempts to describe the magnetic properties of matter do exist. On the other side, the explanatory approaches show great deficiencies in describing the nature of magnetism. One weak spot is that currently used models, e.g. the model of atomic bar magnets, exclusively focus on ferromagnetism whereas dia- and paramagnetism are ignored. In addition, the established models at school are operating with classical point particles. It its known that this approach cannot serve as an appropriate starting point since the Bohr-van Leeuwen theorem states that all kinds of magnetism do vanish according to classical statistical mechanics. Thus, the problem is that models based on point particles are not applicable on higher levels of complexity in a self-consistent manner, which causes problems for students' conceptual growth. Our approach is setting in with simple attraction- and repulsion-experiments based on dia- and paramagnetism, which can be realized at school using low-priced high-energy neodymium magnets only recently. The approach enables us to supply separated clarifications for the orbital contributions (diamagnetism) and the spin contributions (paramagnetism) of non-interacting electrons. Merging these insights ferromagnetism can be introduced using a model based on a visualization of quantum states showing the spin and orbit contributions of interacting electrons.

\section{Concept and Implementation}

Our aim is to develop a self-consistent model that can be extended in complexity from elementary school to high school and finally university level. To ensure the applicability on different levels of complexity the design process is following a top-down design-based research approach. Thus, it is necessary to develop a self-consistent model providing explanations for all three major kinds of magnetism initially at university level, which is the focus of the present contribution. Subsequently, the model needs to be adjusted for high school and elementary school. Our model uses appropriate visualizations and animations together with mathematical formalism of quantum theory at university level. For physics education at school it is sufficient to limit to visualizations thus reducing the complexity of our model.

\section{Objectives and Assessment}

We introduce a self-consistent model using visualizations based on quantum physics to describe and explain dia-, para- and ferromagnetic phenomena at university level. Our approach serves as a starting point for a discussion and possible extension of the current treatment of ferromagnetism in elementary and high schools physics education to dia- and paramagnetism. On this ground, a new and advantageous explanation of magnetism beyond the phenomenological approach becomes accessible based on quantum theory. 


\title{
INVITED SYMPOSIUM IWB:
}

\section{PERSPECTIVES ON INTERACTIVE WHITEBOARD USE IN PHYSICS INSTRUCTION \\ Bor Gregorcic (Chairperson), Uppsala University, Sweden Ian Lawrence (Discussant), Institute of Physics, United Kingdom}

The interactive whiteboard (IWB) has become a widely spread instructional tool, particularly in primary and high-school classrooms in the western world. However, using the IWB in a way that would take advantage of its advanced possibilities for teaching physics still remains a challenge. The symposium will bring together international researchers that have dealt with IWB use in physics instruction. Talks will address the topic of using the IWB in an interactive way in diverse circumstances and from different perspectives.

\section{Interactive Whiteboards as a Means of Supporting Students' Kinesthetic Engagement and Collaborative Inquiry in Physics Bor Gregorcic Uppsala University, Sweden}

\author{
Integrating IWB in Science Laboratory: \\ Opportunities for Improving Science Instruction \\ Giacomo Bozzo ${ }^{1}$, Carme Grimalt-Alvaro ${ }^{2}$, Víctor López Simón \\ ${ }^{1}$ Physics Education Research (PER) Group - UNICAL, Italy, \\ ${ }^{2}$ Centre for Research of Science and Mathematics Education (CRECIM), UAB, Spain
}

\section{Conceptual Modelling Using the IWB in the Field of Kinematics Stefano Vercellati, Marisa Michelini University of Udine, Italy}

\author{
Virtual Reality Experiments for Interactive Whiteboards and Tablets \\ William Lindlahr and Klaus Wendt \\ Johannes Gutenberg University Mainz, Germany
}


Invited Symposium IWB

Room B (D Z005)

Contribution 1B1

Wed, 9 Sep, 11:30-11:45

\title{
Interactive Whiteboards as a Means of Supporting Students' Kinesthetic Engagement and Collaborative Inquiry in Physics \\ Bor Gregorcic \\ Uppsala University, Sweden
}

\begin{abstract}
We will present a conceptual framework for the study and design of instructional activities that draws on the affordances of interactive whiteboards (IWBs) for physics instruction in a social setting. We see the IWB as allowing interplay of kinesthetic and social interactivity, drawing on embodied and socially distributed cognition as the theoretical underpinnings of our research and design of instructional activities.

We will present the findings of a case study where our designed instructional materials were put to use in practice. The study investigated high-school students using the IWB in combination with Algodoo software to investigate orbital motion of planets (Kepler's laws). We were particularly interested in how students combine different semiotic modes (speech, gesture and the virtual environment) to communicate among each other. The students engaged in activities that resemble scientific inquiry and spontaneously drew on a rich variety of semiotic resources spanning different modalities in their discussion, including speech and gesture. This allowed them to engage in discussion and collaborative inquiry without being familiar with the advanced vocabulary and mathematics typically associated with orbital motion. This illustrative case study can help us see how the IWB can be used to support the learning of physics content and the practice of science through hands-on activities and guided collaborative inquiry in topics that traditionally lack hands-on experiments.

The goal of the study was to identify and discern different ways of student engagement and communication when they use the IWB for the purpose of inquiry in order to test and further develop the theoretical framework for the study and design of IWB-based activities.
\end{abstract}




\author{
Integrating IWB in Science Laboratory: \\ Opportunities for Improving Science Instruction

\title{
Introduction and Theory
}

The international literature in education has showed some of several advantages introduced by using interactive whiteboard (IWB) in teaching and learning activities, in terms of pupils' motivation, attention and behaviour. In particular, several studies have demonstrated that the activities performed employing IWB are more attractive for teachers and students than using others classroom educational tools, since IWB is suited to stimulate the interaction between students or between teacher and learners (Higgins et al. 2007).

Nevertheless, different studies conducted in various European countries have also showed that most of teachers in primary and secondary schools do not exploit completely the potential value of interactive whiteboard from pedagogical point of view (Hermans et al. 2008). This problem has stimulated various studies that aimed to shed light on the causes of the underuse or inappropriate use of IWB and has motivated different researchers in education to deal with this important challenge. The analysis of the related literature has led us to focus our attention on three interesting perspective of investigation that are particularly relevant in our research context (Bozzo et al. 2014): (a) The first is related to the Pedagogical Approaches that teachers use when they plan/perform the educational activities that can be conducted by using the IWB; (b) The second is focused on the technical abilities/familiarity of teachers with IWB that influence their attitude to employ this kind of technology in classroom everyday activities (Glover \& Miller 2001); (c) The third regards teachers' beliefs/ideas about the potential value of IWB from a pedagogical point of view (Mama \& Hennessy 2013). Furthermore, although several studies have demonstrated the affordances introduced by IWB in teaching and learning processes, few researches have analysed the contributions of this educational tool in the context of science laboratories. This lack of studies has led us to analyse different activities performed using IWB in a physics ICT-equipped science laboratory from the viewpoint of the three perspectives of investigation above introduced, in order to characterise the affordances of IWB in this specific context.

\section{Concept and Implementation}

We planned this research in order to analyse and to classify the activities that can be performed through the interactive whiteboard in science laboratories. In particular, we videotaped 20 sessions of experimental physics activities performed in the science laboratory of CRECIM Centre (Autonomous University of Barcelona), where different science teachers used IWB with their secondary school students in an ICT-equipped laboratory. We classified the video-clips by using different dimensions of analysis, which allowed us to characterise the practices carried out through the interactive whiteboard in a specific non-formal science laboratory.

\section{Objectives and Assessment}

The main goal of this study was to investigate how interactive whiteboard blends in an inquiry-based science laboratory, which learning opportunities it offers to improve science instruction (in particular to improve physics education) and which role it plays in this specific context. The obtained results allowed us to identify the benefits of IWB for teaching and learning activities performed in an ICT equipped educational science laboratory. Moreover, this analysis offers us the possibility to identify different specific IWB practices that can produce results unattainable by using only traditional educational tools (such as whiteboard, computer and projector). 


\title{
Conceptual Modelling Using the IWB in the Field of Kinematics Stefano Vercellati and Marisa Michelini \\ University of Udine, Italy
}

\begin{abstract}
Introduction and Theory
Interactive whiteboards (IWBs) provide new potentials in science education offering more efficiency to the routine strategies by creating environments in which the sharing of interpretative ideas is promoted (Ilyas and Al - Tabtabaie 2004) and those ideas will become a resource for the development of the process of formal thinking (Michelini \& Vercellati 2014). IWB has the potential to support dynamic cooperation, building new cognitive skills related to visual and perceptual aspects and providing the possibility to track the development of students' representation of models. In this way, the history of students reasoning (often evoked as the basis for the progress in learning) can be documented and analyzed to individuate the peculiar dynamics of the learning process. The students process of bridging from naïve representations of single student model(s) of physical phenomena to scientific interpretative models constructed by the class community becomes a path of reasoning that could be re-analyzed by the community itself (Robinson, 2008).
\end{abstract}

\section{Concept and Implementation}

This work is the implementation of a module of formative intervention for secondary school students in which the IWB is adopted to create a context in which students, following an activity of Conceptual Modelling (Michelini \& Vercellati, 2014) has to develop a formal interpretation of the kinematic of simple motions starting from the analysis of experimental situations realized in class.

Using a webcam, students recorded motions and visualize them on the IWB to have the possibility to draw on the real acquired situation by sharing and discussing their naïve representation of motion. Then, through the in-class discussion, they provide shared model(s) that will be tested transporting them on simulated situations (using Algodoo) and then compare with the usual description provided by Tracker.

\section{Objectives and Assessment}

Data were collected by recording the IWB screen and the in-class discussion with the aims to document and to analyze the process that drove students to develop their shared model(s) for the description of motion.

\section{Bibliography}

Glover D, Miller D, Averis D and Door V (2005). The interactive whiteboard: a literature survey. Technology, Pedagogy and Education 14, 2, $155-170$.

Ilyas M A B and Al - Tabtabaie A M (2004). Improving Knowledge Delivery and Information Retention through 'Smarter' Interactive Whiteboards, Proc. of International Conference on Computers in Education 2004.

Michelini M, Vercellati S (2014). The IWB as a bridge between phenomena exploration and interpretation of electromagnetic phenomena in construction of formal thinking, MPTL18 Madrid 11-13 September 2013

Robinson A (2008). Easy Implementation of Internet - Based Whiteboard Physics Tutorials. The Physics Teacher, 46. 
Invited Symposium IWB

Room B (D Z005)

Contribution 1B4

Wed, 9 Sep, 12:30-12:45

\author{
Virtual Reality Experiments for Interactive Whiteboards and Tablets \\ William Lindlahr and Klaus Wendt \\ Johannes Gutenberg University Mainz, Germany
}

\begin{abstract}
Virtual Reality Experiments (VREs) simulate advanced high school experiments of physics in a 3D-environment. Conducting VREs enables students to perform virtually those experiments, which would otherwise not be accessible in school or high school due to various restrictions, i.e. because of financial nature, potential hazards or too high experimental effort. The Virtual Reality Experiments designed by us aim for an accurate reproduction of the corresponding real experiments, precisely simulating all individual conditions and controls. At the same time they allow for a high degree of freedom for both, teachers and students, in terms of execution as well as supervision. In addition, the software provides supplementary, didactically encouraging visualizations related to the experiment.

The computer simulations emulate experimental setup and functionality in perfect reproduction of the reality while considering further didactic principles. For instance, the virtual devices correspond to modern instruments as used in real state of the art experiments, or alternatively exhibit the original historical apparatus. Furthermore, similar as in the real experiments, the measurements are error-prone, leading to limited precision, statistics and uncertainties. Consequently, the virtually measured data sets realistically represent measured values of real experiments.

In general, all options of interaction in the VRE reproduce the operation mode of the real device by providing a regular and most identical (natural) user interface. Similar to the operation of the real experiment, the user is able to press buttons, to turn knobs and to connect electronics by using manual control through his fingers on the touch screens. As result, complete flexibility for performing individual experiments is achieved, which is only limited by didactic considerations. For example, the user may not destroy the VRE experimental devices no matter if accidentally or on purpose.

The VREs' realistic presentation and the options for interaction are complemented with additional visualizations in the virtual space, which aim for supporting and facilitating the process of understanding of the experiments and the corresponding physical concepts. For instance vectors of force, directions of current or tracks of individual particles may be displayed, which react dynamically to represent parameters in space and time. In this way employing augmented reality, these visualizations can be switched on and off by teachers or students.
\end{abstract}




\title{
INVITED SYMPOSIUM VRL:
}

\author{
VIRTUAL AND REMOTE LABS IN PRACTICE \\ Lars-Jochen Thoms (Chairperson), LMU Munich, Germany \\ David Lowe (Discussant), University of Sydney, Australia
}

If expensive, complex, or challenging experimental set-ups are not available or practicable, virtual and remote labs offer new, additional possibilities, which enable experimental experience, develop experimental competencies, and furthermore assist discovery learning. For many reasons, experiments should be feasible in distance learning. Virtual and remote labs enable students to conduct demonstration experiments in a self-responsible, active and at the same time harmless fashion. In this symposium we will discuss latest trends in researchbased development of virtual and remote labs as well as long-time experiences in the use of virtual and remote labs in distance learning.

\section{Experimenting From a Distance: Optical Spectrometry via the Internet Lars-Jochen Thoms and Raimund Girwidz LMU Munich, Germany}

\author{
Virtual and Remote Laboratories on Optics in a Moodle Course \\ Luis de la Torre, Juan Pedro Sánchez, Jacobo Sáenz, Carmen Carreras, \\ Manuel Yuste, Jose Sánchez and Sebastián Dormido \\ UNED, Spain
}

Implementing Different Learning Goals in an Online Experiment Stefan Richtberg and Raimund Girwidz LMU Munich, Germany

Improving Undergraduate Engagement with Online Labs: Student Priorities and Strategies for Virtual and Remote Investigations Marcus Brodeur, Nicholas Braithwaite, Ulrich Kolb and Shailey Minocha The Open University, UK

\author{
Breaking Barriers: \\ Working with Students as Partners to Develop Innovative Virtual Laboratories \\ Sam J. Nolan \\ University of Durham, United Kingdom
}

\author{
Inquiry Based Remote Controlled Labs \\ Jan Mulder, Piet Blankert and Gerrit Kuik \\ Vrije Universiteit Amsterdam, Netherlands
}




\title{
Experimenting From a Distance: Optical Spectrometry via the Internet Lars-Jochen Thoms and Raimund Girwidz LMU Munich, Germany
}

\begin{abstract}
Introduction and Theory
Many national science education standards define competencies in science and engineering that all students should be able to demonstrate at subsequent stages in their K-12 learning experience (e.g., National Research Council, 2013). Experimenting is an essential part of physics and physics education. Hence, tools are needed that assess both knowledge and skills of knowledge acquisition. Since hands-on experiments are not suitable for large-scale assessment, process-oriented experimental competencies may be assessed in virtual labs. Considering the 2006/7 debate on minimally guided teaching techniques, assessment tools on experimental competencies including process-oriented inquiry techniques should be familiar to students to prevent cognitive overload while assessing competencies. Furthermore, the format of an assessment tool for a particular learning environment should be similar to the format of the learning environment itself. Thus, teachers should introduce theses assessment tools in class as instructional tools.
\end{abstract}

\section{Concept and Implementation}

Banchi and Bell (2008) proclaim four levels of inquiry in activities with rising opening and withdrawal of predefined structure: confirmation inquiry, structured inquiry, guided inquiry and open inquiry. Based on this classification, we developed a remotely controlled laboratory with predefined set-ups selectable according to the class. This reduces the complexity of the lab activity for students. Students can carry out real experiments via the Internet. They can choose from different light bulbs and analyze them with a spectrometer. All user activities are logged by the system without disturbing the students.

\section{Objectives and Assessment}

Assessment of experimental competencies is not yet well established. We just started with an empirical pilot study, too. This study aimed to examine if undergraduate students may successfully use a predefined remote lab activity to introduce atomic physics to themselves on their own. We evaluated the experimental setup and the accompanying worksheet with $N=244$ students in a laboratory condition.

Since our local curriculum preserves approximately two 45-minute lessons for the introduction to atomic physics especially including the introduction to atomic spectra, excitation, de-excitation, quantized energy radiation, and term-schemata. Even if the school owns a spectrometer, there is not enough time to include a lab activity in class. A homework-based activity in a remote lab can fill this gap and may deliver a new approach to procedural learning. Furthermore, lab activity as homework can foster problem-based, discovery, and inquiry-based learning and support collaborative and cooperative learning as well.

Technical details and the experimental potential are explained as well as some findings from this empirical pilot study about the learning scenario mentioned.

\section{References}

Banchi, H., \& Bell, R. L. (2008). The Many Levels of Inquiry. Science and Children, 46, 26-29.

National Research Council. (2013). Next Generation Science Standards: For States, By States. Washington, DC:

The National Academies Press. 
Invited Symposium VRL

Room A (D Z003)

Contribution 2A2

Wed, 9 Sep, 14:20-14:35

\author{
Virtual and Remote Laboratories on Optics in a Moodle Course \\ Luis de la Torre, Juan Pedro Sánchez, Jacobo Sáenz, Carmen Carreras, \\ Manuel Yuste, Jose Sánchez and Sebastián Dormido \\ UNED, Spain
}

\title{
Introduction and Theory
}

Virtual and remote labs are tools that support distance and blended learning in science and engineering courses. The former are computer based simulations which offer similar views and ways of work to traditional hands-on laboratories while the latter use real setups which can be used at distance.

Since their appearance, developing and deploying these tools has been a complex issue, out of reach for teachers who are experts in their field but lack the numerous skills that have been historically required to create a virtual and/or remote lab and then publish it on the web.

This work presents an online course with four virtual and remote laboratories on optics (i.e. a total of eight labs). As much as the development as the deployment of these tools is made very easy thanks to the methodology and tools used for these purposes.

\section{Concept and Implementation}

Virtual and remote laboratories are created using EjsS. EjsS stands for Easy Java/Javascript Simulations and it is a free and open source tool. It was originally designed for making the creation of physics simulations very easy but it has been growing and changing since its birth. Nowadays, EjsS is used for creating any type of simulations, virtual laboratories and, since connections with a broad wide variety of hardware and control software are now supported, even remote laboratories.

While the creation of the virtual and remote laboratories is made as simpler as possible thanks to the use of EjsS, their deployment (and subsequent management) is done through the use of Moodle. Moodle is a free and open source learning management system, created to help learners with their learning and teachers with their teaching. By using this tool and a few plugins developed by the authors, virtual and remote labs can be published in online courses in an extremely easy way.

\section{Objectives and Assessment}

The work presents an online course with eight labs: four virtual and four remote. There are four different experiments on optics (light in isotropic media, focal length of a thin lens, Fraunhofer diffraction and the photoelectric effect), each of them covered by a virtual and a remote lab. Both versions complement each other, offering different insights of the same experiment to the students and providing them with theoretical results (virtual labs) as well as with real results (remote labs).

The presented course and labs have been in use during the course 2014-2015 by the students of the Experimental Techniques in Physics III subject in UNED, which is an open university and the largest one in Spain in terms of number of students. 


\section{Implementing Different Learning Goals in an Online Experiment \\ Stefan Richtberg and Raimund Girwidz \\ LMU Munich, Germany}

\section{Introduction and Theory}

Experiments are important parts of Physics classes. In Germany three quarters of a lesson are filled with activities around experiments, like discussing the experimental setup, performing experiments or evaluating generated data (Tesch, 2005). Teachers pursue different learning goals when using experiments, for example showing the connection between theory and experiment or using new methods of science thinking (Wetzel et al., 1998). But these goals are often limited when an experiment can be performed as demonstration only. Online experiments can reverse these limitations and focus equally on different learning goals. The developer can provide special tasks, can sequence the experiment, can provide scaffolding in different forms and can present feedback based on user actions.

\section{Concept and Implementation}

So we developed a browser-based learning environment about the electron deflection in electric and magnetic fields. This environment contains two virtual experiments where students can perform experiments, generate data and evaluate this data individually. Thereby the students are supported by different scaffolds, by feedback and by the use of virtual reality. To cover two typical learning goals - showing connection between theory and experiment and evaluating data in an appropriate way - we build two learning paths. One path is focused on forces and equations of motion. Here the experiment is used to check results and to confirm the generated theory. The other path uses the experiment to generate data. This data is used to build an appropriate theory.

\section{Objectives and Assessment}

In a study with 80 students we considered the question whether students use the virtual experiment in the expected way and whether they check hypotheses with the experiment in a scientific way. Moreover we analyzed the support components students need when generating the formula of the electron path. Also we checked their knowledge in different domains after using the learning environment. The talk gives a short summary of the theoretical background and technical challenges, presents main parts of the environment and discusses some results of our studies and our conclusions for developing virtual experiments. 
Invited Symposium VRL

Room A (D Z003)

Contribution $2 \mathrm{~A} 4$

Wed, 9 Sep, 15:00-15:15

\author{
Improving Undergraduate Engagement with Online Labs: \\ Student Priorities and Strategies for Virtual and Remote Investigations \\ Marcus Brodeur, Nicholas Braithwaite, Ulrich Kolb and Shailey Minocha \\ The Open University, UK
}

\title{
Introduction and Theory
}

Online laboratories have seen expanding use by postsecondary institutions as preparation or, occasionally, replacement - for scientific investigations typically conducted as 'hands on' practical work. Recent studies indicate that computer-mediated approaches to experimentation can achieve similar, if not superior, outcomes for students when compared with more traditional lab-based activities. Still, there remains considerable skepticism by undergraduates asked to supplement (or supplant) 'on site' science education with these tools. If we accept that such skepticism negatively impacts engagement with online experiments - and students' ability to derive full instructional benefit from them - then it behooves us to find ways to ameliorate student mistrust of online labs. The approach taken by this study is to elicit the preconceptions and priorities held by undergraduates towards practical science work and thereby identify which aspects of online labs must be altered in order to achieve the best possible pedagogical outcomes.

\section{Concept and Implementation}

A large-scale study $(\mathrm{N}=1140)$ of science undergraduates was undertaken at the Open University [UK], comprising a larger set of second-year students studying practical science topics across five scientific disciplines and a smaller set of third-year students working on dedicated astrophysics content. As both cohorts made extensive use of online labs in the form of virtual and/or remote experiments (e.g., live operation of a robotic telescope over the internet), multi-stage surveys and end-of-module group interviews were conducted to elicit feedback on their experiences with this approach to practical science. Likert, ranking and open-ended questions permitted broadly universal sentiments of online experimentation - as well as key differences of opinion - to be identified and correlated with student performance on related assessments. Thematic coding of the interview comments provided explanatory power and support for the trends observed in the quantitative data.

\section{Objectives and Assessment}

The principal objectives of this study were to determine to what extent three key aspects of computer-mediated practical work - authenticity, sociability and metafunctionality - influenced students' ability to successfully acquire practical techniques and skills through remote and/or virtual experimentation. Briefly it was found that, concerning online lab experiences, undergraduates place considerably greater value on professional relevance and data reliability than on realism; they crave greatly expanded provisions for synchronous social modes of learning over what currently exist; and they feel troubleshooting scenarios are currently of greater value than sensory augmentation. 


\section{Breaking Barriers: Working with Students as Partners to Develop Innovative Virtual Laboratories Sam J. Nolan \\ University of Durham, United Kingdom}

\section{Introduction and Theory}

As every educator learns from experience, teaching a subject deepens our understanding and appreciation of it. Therefore many see the creation of student generated learning content as essential in enhancing the student's learning experience. However there are many risks associated with this approach e.g. Will the content be appropriate? Will the content be accessible? How will the student react if the materials are criticised?

One particular issue which has been noted at Durham University was that in conducting experiments students are often concerned with the technical aspects of the experiments they are undertaking, whilst not considering the deeper underlying scientific mechanisms at play [1], this has also been notes in work done at the secondary education level [2]. To address these surface learning approaches and working with summer student developers (themselves veterans of the laboratory classes) we have sought to develop innovative e-learning solutions (including interactive screen experiments [3]), that students can undertake as prelaboratory tasks to aid them in increasing their familiarity with data taking and hence help them to spend a larger fraction of their time in the laboratory engaging with the physics behind the experiments they're undertaking.

\section{Concept and Implementation}

In this paper, we'll look at how this was implemented through 3 case studies of studentgenerated multimedia content in physics at Durham University:

1. In the first case study students who are 'veterans' of the laboratory class are employed to develop interactive screen experiments to be used as pre-laboratory tasks.

2. In the second case study summer undergraduate students are tasked with developing partially augmented reality systems to enhance the visualisation skills of experimental physics and chemistry undergraduates.

3. In the final case study we will discuss how through the development of a photograph based virtualisation of the Bell's Inequalities experiment we've allowed the greater inclusion of experimental discovery in the teaching of quantum mechanics to third year undergraduate students.

\section{Objectives and Assessment}

All three case studies have an overarching objective to deepen student learning and conceptual understanding of physics. This was assessed through qualitative interviews with staff demonstrators and students discussing their experiences of learning prior to and after the introduction of the multimedia content. As we will discuss these indicated an overwhelmingly positive response to the materials. This was also backed up by stronger engagement with the materials developed year on year. These materials are available as OER and have been picked up and utilised by several Universities and further education institutes.

\section{References}

1. S.J. Nolan, M. Szablewski, T.J.L. McComb and I.G. Hughes: (2013) 'Using Interactive Screen Experiments as Pre-Laboratory Tasks to Enhance Student Learning'. New Directions 9(1), 24-27.

2. Hofstein, A. \& Kind, P.M. (2012). Learning in and from Science Laboratories. In Second International Handbook of Science Education. Eds. Fraser, B.J., Tobin, KG. \& McRobbie, C.J. Dordrecht: Springer. 189-207.

3. Kirstein, J. and Nordmeier, V. (2007) Multimedia representation of experiments in physics. European Journal of Physics, 28, S115. 
Invited Symposium VRL

Room A (D Z003)

Contribution $2 \mathrm{~A} 6$

Wed, 9 Sep, 15:40-15:55

\author{
Inquiry Based Remote Controlled Labs \\ Jan Mulder, Piet Blankert and Gerrit Kuik \\ Vrije Universiteit Amsterdam, Netherlands
}

\begin{abstract}
In upper level high school in the Netherlands students have to conduct a few advanced experiments (typically 8-15 hours per experiment) and a small research project with a workload of 80 hours. Students are encouraged to consult experts outside school and regularly visit universities for assistance.

To accommodate some of these high school students a number of remote controlled labs (RCLs) are available that allow students to conduct inquire based experiments. These RCLs allow students some freedom in defining research questions and arrangement of the experiment. The experiments do require some preparation: the physics concepts are typically dealt with in high school but the equipment is too advanced for high schools. For research projects students are expected to explore a topic beyond high school level. Along with the RCLs educational materials have been prepared to guide students through self--study. The RCLs are suitable as advanced experiments as well as for small research projects for high school students. The same set of RCLs is also available to first and second year bachelor students in physics. With alternative interfaces to control the experiments, bachelor students further are given more freedom in setting up their experiment, allowing more challenging tasks to be undertaken.

In the presentation a few examples of RCLs will be illustrated, along with the educational support materials prepared for high school students. How the same RCLs are incorporated in undergraduate labs will also be discussed. For a broader overview and some real experiences we kindly invite participants to join our workshop on RCLs.
\end{abstract}




\title{
INVITED SYMPOSIUM ILA:
}

\section{CONCEPTS TO INITIALIZE LEARNING ACTIVITIES WITH MODERN MEDIA \\ Tomasz Greczyło (Chairperson), University of Wrocław, Poland \\ Ian Lawrence (Discussant), Institute of Physics, United Kingdom}

The symposium Concepts to Initialize Learning Activities with Modern Media is going to make bases for creating a platform to share ideas and examine the topic of Modern Media in Physics Teaching and Learning broadly as well as present various points of view. The presentations within the symposium will be treated as introduction to discussion. The input will be critically reviewed during the event which should result in specific proposals.

\section{Concepts to Initialize Learning Activities With Modern Media Tomasz Greczyło and Ewa Dębowska University of Wrocław, Poland}

\author{
Physics in Movies: A Way for Teaching? \\ Alexander Strahl and Markus Herbst \\ University of Salzburg, Austria
}

\footnotetext{
Animation With Algodoo: A Simple Tool for Teaching and Learning Physics Samir L. Da Silva ${ }^{1}$, Judismar T. Guaitolini Junior ${ }^{1}$, Emilson R. Viana ${ }^{2}$, Rodrigo L. Da Silva ${ }^{3}$ and Fábio F. Lea ${ }^{3}$

${ }^{1}$ Instituto Federal do Espírito Santo, Brazil, ${ }^{2}$ Universidade Tecnológica Federal do Paraná, Brazil, ${ }^{3}$ Instituto Federal Fluminense, Brazil
}

The Physics Experiments Based on Sensor and Computer Yun WANG and Qinyun MENG Beijing University of Chemical Technology, China

\author{
iRadioactivity: \\ Using Smartphones and Tablet PCs as Radioactive Counters in Physics Education \\ Alexander Molz $z^{1}$, Jochen Kuhn ${ }^{1}$ and Andreas Müller ${ }^{2}$ \\ ${ }^{1}$ University of Kaiserslautern, Germany, ${ }^{2}$ University of Geneva, Switzerland
}

\author{
gPhysics: Using Google Glass as Experimental Tool for \\ Wearable-Technology Enhanced Learning in Physics \\ Jochen Kuhn ${ }^{1}$, Paul Lukowicz ${ }^{2}$, Michael Hirth' ${ }^{1}$, \\ Andreas Poxrucker ${ }^{2}$, Jens Weppner ${ }^{2}$ and Benjamin Bischke \\ ${ }^{1}$ University of Kaiserslautern, Germany, \\ ${ }^{2}$ German Research Center for Artificial Intelligence (DFKI) Kaiserslautern, Germany
}




\section{Concepts to Initialize Learning Activities With Modern Media \\ Tomasz Greczyło and Ewa Dębowska \\ University of Wrocław, Poland}

\section{Introduction and Theory}

Modern Media understood as all types of diversified information and communication technologies (D-ICT) that are intended to reach a large audience by specified communication involving different channels of influence and pointing to different ways of perception are considered as powerful and attractive collection of tools and methods that enrich educational resources of educators and teachers. Five characteristics of mass communication [1] that could, after small redefinition, be also assigned to D-ICT, have been identified. They:

- comprise technical and institutional methods of production and distribution (from paper to mobile applets),

- involve the commodification of symbolic forms, as the production of materials relies on its ability to manufacture and distribute (including selling) large quantities of the institutional work (for example science centres rely on their workers and scientific equipment),

- separate contexts between the production and reception of information,

- reach those 'far removed' in time and space, in comparison to the producers (making use of variety of communication platforms),

- distribute information - a 'one to many' form of communication, whereby products are mass-produced and disseminated to a great quantity of audiences (via institutional and social network).

The characteristics will be identified in presented concepts to initialize learning activities.

\section{Concept and Implementation}

A group of physicists associated with the MPTL - international conference on Multimedia in Physics Teaching and Learning (since 1996) - performs the reviews resulting in the recommendation of ICT materials useful in preparation of activities devoted to different subject areas in physics [2]. A broad number of institutions which are creating and offering learning activities run different projects and make extensive and often unique use of modern media. Based on their own and sheared experiences a process of optimization is taking place therefore use of modern media becomes necessary ingredient in teaching and learning processes. A discussion within a group of authors-experts could give birth to new concepts and make the use of modern media more efficient and successful.

\section{Objectives and Assessment}

The symposium is going to make bases for creating a platform to share ideas and examine the topic broadly as well as present various points of view on the modern media. The subject will be introduced to initialize discussion. The input will be critically reviewed during the event which should result in specific proposals.

\section{References:}

1. J. B. Thompson, The Media and Modernity: A Social Theory of the Media, Stanford University Press, California 1995

2. E. Dębowska, R. Girwidz, T. Greczyło et al. Report and recommendations on multimedia materials for teaching and learning electricity and magnetism, Eur. J. Phys. 34 (2013) L47-L54 


\section{Physics in Movies: A Way for Teaching? \\ Alexander Strahl and Markus Herbst \\ University of Salzburg, Austria}

\section{Introduction and Theory}

One of the most frequently asked requirements of physics at school is to include the "everyday physics". This can be achieved by the analysis of physical movie scenes. Dealing with physical content in this way can highly motivate students and make them deliberate, if specific movie scenes can actually take place in real life in order to train their critical approach to media. This is connected in the field of authentic context (Kuhn, 2010).

\section{Concept and Implementation}

The capabilities and the variety of implementation regarding films in physics at school will be shown. Some examples will point out what content in movies could have an effect on students in increasing their knowledge and motivation. For example, the scene "destruction of death star one" (Star Wars Ep. IV: A new Hope; Lucsafilm) implies four different physical contexts (e.g. Doppler shift, etc.) to analyze.

\section{Objectives and Assessment}

The concept of using movies to teach physics is nothing new. It was originally formed in a lecture for physics at university and was soon transferred into school classrooms (Strahl, Mennerich, Süllow \& Müller, 2007; Dammaschke \& Strahl, 2010).

This presentation deals with the analysis of different movie scenes and points out their possibilities for practical use.

\section{References}

Kuhn, J. (2010). Authentische Aufgaben im theoretischen Rahmen von Instruktions- und Lehr-Lern-Forschung: Effektivität und Optimierung von Ankermedien für eine neue Aufgabenkultur im Physikunterricht. Wiesbaden: Vieweg+Teubner Verlag.

Strahl, A., Mennerich, C., Süllow, S., Müller, R. (2007). Physics made by Hollywood: Über den Einsatz von Unterhaltungsfilmsequenzen in der Physik-Lehre. Praxis der Naturwissenschaft Physik - Heft 7/56, Okt 2007.

Dammaschke, T. , Strahl, A. (2010). Physik in anderen Welten. Literatur, Film und Fernsehen für das Lernen von und über Physik nutzen. Unterricht Physik: Physik in fiktionalen Medien. p. 4-9, Heft 120, Dez 2010. 


\author{
Animation With Algodoo: A Simple Tool for Teaching and Learning Physics \\ Samir L. Da Silva ${ }^{1}$, Judismar T. Guaitolini Junior ${ }^{1}$, Emilson R. Viana ${ }^{2}$, \\ Rodrigo L. Da Silva ${ }^{3}$ and Fábio F. Leal ${ }^{3}$ \\ ${ }^{1}$ Instituto Federal do Espírito Santo, Brazil, ${ }^{2}$ Universidade Tecnológica Federal do Paraná, Brazil, \\ ${ }^{3}$ Instituto Federal Fluminense, Brazil
}

\title{
Introduction and Theory
}

In the last years, several teachers have presented a good pedagogical approach by using numerical experiments of low computational cost, to minimize the difficulties of teaching and learning a physical phenomenon. They propose strategies to elaborate good tools for supporting the cognitive development of the students. Such technologies have been widely used in the physics teaching.

However, the resources in terms of people, space, and finances are a major concern in many institutions today, especially in underdeveloped countries. Often leading to the emergence of overcrowded classes, difficulties of acquiring material resources to perform experimentation, simulations and animations. One solution is to design and build low-cost tools that minimize preparation time by the teacher and maximize learning.

\section{Concept and Implementation}

In this work, we use the free software for 2D simulation known as Algodoo of Algoryx Simulation $\mathrm{AB}$. This software has an interactive environment, allowing creation of experimental scenarios, as animated movies, but having as feedback, the equations and physical properties implemented to the simulations. The Algodoo is an easy manipulation tool, and does not require specific knowledge about computer programming or training to realize tasks in the software, which allow easy learning by students and easy implementation for teachers. Thus, more time can be devoted to the basics of learning through visualization and interaction with the animation instead spending a long time modelling the problem with more complicated programming languages. The art of teaching physics using this freeware can be applied to students of different ages and level of education, from high school to college students. The Algodoo provides the study of several physical systems creatively and interactively.

\section{Objectives and Assessment}

These animations can be used in several degree levels in school. For high school students, we can focus on the physical concepts and the qualitative analysis, without a mathematical rigor. But, for undergraduate students the animations also enhance the comprehension of the mathematics procedure involved in the analysis of the system. In addition, for college students, they can learn the scientific procedures of how to model physical systems. 


\section{The Physics Experiments Based on Sensor and Computer \\ Yun Wang and Qinyun Meng \\ Beijing University of Chemical Technology, China}

Albert Einstein said on education: '...Knowledge is dead; the school, however serves the living. It should develop in the young individual those qualities and capabilities which are of value for the welfare of the commonwealth....

College physics is an important basic course for the students of science and engineering in college. To resolve the problems of obsolete content, underdeveloped ways in the general physics experiment teaching, this paper discusses the information reform of general physics experiment teaching and the necessity of reform measures.

For the goal of training students' knowledge structure, ability structure and quality structure, in order to shape students subjectivity active and creative, we formed a new experimental teaching curriculum system which emphasized basic ability training, step by step, focusing on innovation, learning to study.

Some processes are too fast, for example, conductive rubber transformed from the conductor into the insulator when it is heated, some processes cost too long a time, for example, to monitor the power stability of gas laser, that could not be measured by naked eye. Based on sensor and compute technology, we developed data acquisition devices to measure, analysis and control the temperature, force, mass, humidity, voltage pressure, vacuum, combustion, flow, level, viscosity, density, concentration, acidity, rotation and so forth. These variables were displayed in real time, and more conveniently, more accurately. Some physics phenomena were also demonstrated more impressively. For example, Measurement of Liquid Surface Tension became to an interesting experiment, yet it was considered too simple before.

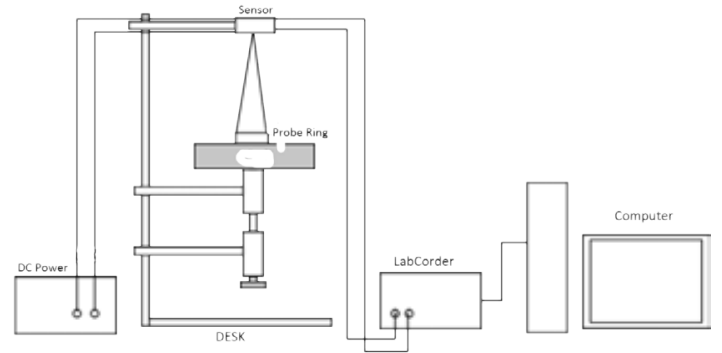

Figure 1. Illustration of the devices.

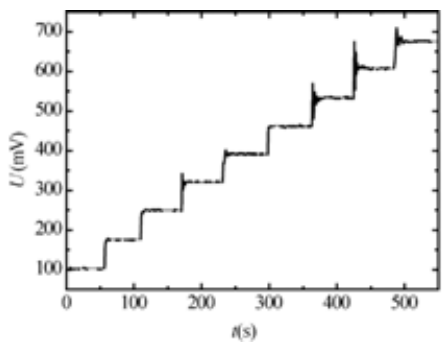

Figure 2: Sensor's voltage changed with time in calibration.

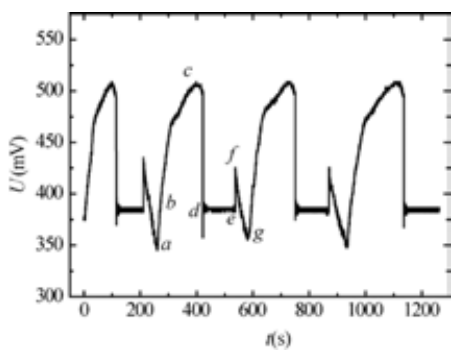

Figure 3. Sensor's voltage changed with time in measurement.

Along with its flexible, vivid, animate, and colorful movie, image, text, voice and picture forms, computer assisted instruction also applied in physical experiments, to change the content from difficult to easy, static to dynamic, abstract to concrete. The human-computer interaction and information sharing developed the student's field of vision and creative thinking ability. 


\author{
iRadioactivity: \\ Using Smartphones and Tablet PCs as Radioactive Counters in Physics Education \\ Alexander Molz ${ }^{1}$, Jochen Kuhn ${ }^{1}$ and Andreas Müller ${ }^{2}$ \\ ${ }^{1}$ University of Kaiserslautern, Germany, ${ }^{2}$ University of Geneva, Switzerland
}

\title{
Introduction and Theory
}

Mobile devices, especially smartphone and tablet PC, have increasingly become everyday tools, particularly for the younger generation. In recent years, various articles have been published about the use of smartphones and tablet PCs as experimental tools especially in physics classroom. This is possible because today's smartphones and tablet PCs are equipped with many sensors, which can be used to perform quantitative measurements of for example sound, acceleration, light intensity, air pressure or temperature. While a majority of articles mainly present experiments on mechanics or acoustics, in which the acceleration sensor or the microphone is used, in this contribution we present several experiments for studying radioactivity using the camera sensor of a tablet PC.

\section{Concept and Implementation}

In this contribution we describe how radiation can be detected by the devices' built-in camera sensors in combination with the "RadioactivityCounter" app. We present a learning sequence "iRadioactivity: Opportunity and risk of ionizing radiation" which gives pupils the opportunity to explore (1) the distance law, (2) the law of absorption and (3) the decay law in a one day learning circuit using smartphones and tablet PCs as radiation detectors. Therefore a certified Sr-90 standard radiation source (activity A $=45 \mathrm{kBq}$; note that this is 10 times the activity level of the typical source in a U.S. physics lab, but similar results should be obtainable with longer data-taking times) is used, which is available in many school settings. All three experiments are implemented on interesting contexts, like Fukushima or cancer therapy and pursue the goal of expanding pupil's radiation literacy. The learning sequence is part of the experimental range of the iPhysicsLab (iPL), the physics school lab of the Technical University of Kaiserslautern where school classes can conduct physics experiments of different subject areas based on the theoretical and conceptual framework of out of school learning places - such as Person-Object-Theory of Interest and Context Based Learning.

\section{Objectives and Assessment}

On the one hand the contribution shows radioactivity as a further field of smartphones and tablet PCs as experimental tools in physics education. On the other hand we study how an appropriate curriculum with smartphones as radiation detectors (that means preparation and post processing activities of the lab visit in physics school lessons) affects learning and motivation. Hypotheses and research questions concerning these variables are presented as well as design and first results of a quasi-experimental intervention-control-group study in pre-post-follow up design (senior class; $\mathrm{n}=80$; average age $=18$ ). 


\author{
gPhysics: Using Google Glass as Experimental Tool for \\ Wearable-Technology Enhanced Learning in Physics \\ Jochen Kuhn ${ }^{1}$, Paul Lukowicz ${ }^{2}$, Michael Hirth ${ }^{1}$, \\ Andreas Poxrucker ${ }^{2}$, Jens Weppner ${ }^{2}$ and Benjamin Bischke ${ }^{2}$ \\ ${ }^{1}$ University of Kaiserslautern, Germany, \\ ${ }^{2}$ German Research Center for Artificial Intelligence (DFKI) Kaiserslautern, Germany
}

\begin{abstract}
Introduction and Theory
Wireless communication and mobile technologies provide opportunities for new interaction approaches. Especially the active wearable computing in general as well as mobile and ubiquitous learning in particular have become dynamic and active research areas in the last years corresponding the technological development. While using Head-Mounted-Displays (HMD) as an experimental tool for physics experiments is really new, this project relates to an extremely dynamic trend in physics education: Using internal sensors of everyday modern communication technology as experimental tools. The rationale of using HMD - such as Google Glass - as experimental tool for physics learning is, first, the possibility of creating and using multiple representations with these devices which are important for (conceptual) learning and solving problems - especially in science education. Second, following the theoretical framework of context based learning, we expect that curiosity is increased by using HMD as experimental tools.
\end{abstract}

\title{
Concept and Implementation
}

In a first pilot study we developed a concept which allows studying acoustic phenomena with Google Glass. Starting with the well-known everyday phenomenon that the tone of a water glass is increasing the more water is filled in, students should study the relationship between the frequency of the sound generated by hitting a water glass and the amount of water that was filled in.

In this setup different interactions between students' data analysis and the detecting device are possible: The fill level could be detected automatically by vision recognition or it could be entered by head motion respectively eye motion of the students themselves. The frequency of the tone is detected by the microphone of the Google Glass and the graph of the frequencyfill level-relationship is displayed directly after detecting the fill level and measuring the tone frequency.

The contribution presents this concept and discusses a quasi-experimental, interventioncontrol-group studies in high school physics: 18 randomly sampled 12th grader high school students examined the fill level-tone frequency relationship equipped with Google Glass while 18 other randomly sampled 12th grader high school students study this with tablet PC. The study was imbedded into regular physics classes and conducted as pre-post-follow up design.

\section{Objectives and Assessment}

Besides the usability and possibility of implementation of this device in physics education, we study the following hypotheses based on the theoretical and conceptual framework mentioned above:

Using HMD as experimental tools will foster curiosity concerning analyzing physics phenomena in acoustics compared to the use of studying the phenomenon with smartphones/tablet PC. We study these dependent variables by using well-established and well-validated paper-andpencil tests. The results showed that the hypotheses could be confirmed significantly and with medium up to large effect sizes. Although the results of the studies are encouraging, more work has to be done with larger sample size and in different topics. 
PARALLEL SESSION QP:

\section{MULTIMEDIA IN TEACHING AND LEARNING QUANTUM PHYSICS}

Visualising the Invisible: The Qubit as a Key to Quantum Physics Wolfgang Dür ${ }^{1}$ and Stefan Heusler ${ }^{2}$

${ }^{1}$ University of Innsbruck, Austria, ${ }^{2}$ University of Münster, Germany

A Learning Path on Quantum Physics Including Simulations, Low Cost Experiments, Online Resources Massimiliano Malgieri, Pasquale Onorato and Anna De Ambrosis University of Pavia, Italy

\section{A Quantum Information Course with Python Simulations for Computer Science Students \\ Sabine Tornow \\ Munich University of Applied Sciences, Germany}

An Interactive On-line Course in Contemporary Physics Dean Zollman ${ }^{1}$, Raiya Ebini ${ }^{1}$ and Ulaş Üstün ${ }^{1,2}$

${ }^{1}$ Kansas State University, USA, ${ }^{2}$ Artvin Çoruh University, Turkey 


\author{
Visualising the Invisible: The Qubit as a Key to Quantum Physics \\ Wolfgang Dür ${ }^{1}$ and Stefan Heusler ${ }^{2}$ \\ ${ }^{1}$ University of Innsbruck, Austria, ${ }^{2}$ University of Münster, Germany
}

\title{
Introduction and Theory
}

We present an approach for teaching quantum physics at high school level based on the simplest quantum system - the single quantum bit (qubit). We show that many central concepts of quantum mechanics, including the superposition principle, the stochastic behavior and state change under measurements as well as the Heisenberg uncertainty principle can be understood using simple mathematics, and can be illustrated using catchy visualizations. We discuss abstract features of a qubit in general, and consider possible physical realizations as well as various applications, e.g. in quantum cryptography. The approach can be expanded to two and more qubits, where entanglement plays a key role. We develop illustrations and visualizations of entangled states and discuss fundamental principles as well as applications. This ranges from Bell inequalities to quantum teleportation. The approach connects directly to modern research themes, and allows one to discuss topics that are associated with the Nobel prize for physics 2012.

\section{Concept and Implementation}

The central idea of the approach is to use the quantum two-level system as a tool to introduce the fundamental concepts of quantum physics. For a treatment at high-school level, we focus on catchy visualizations that allow one to illustrate all basic concepts and ideas. For a treatment at undergraduate university level, we also provide a mathematical description, where in contrast to a standard or historical approach to quantum mechanics based on the wave function, simple mathematics suffices. In particular, only vectors and their manipulation are required.

\section{Objectives and Assessment}

The objective is to promote the power of a qubit approach to quantum physics among highschool and undergraduate teachers, and show that most of the central features of quantum mechanics can be explained and illustrated with a the simplest quantum system. Visualizations are the key element to this approach, where we show that quantum superpositions, as well as the stochastic behavior and state change under measurement can be illustrated and explained. The qubit also provides a unifying framework that is novel to physics education, and allows one to make a number of different quantum scenarios accessible at an introduction level. This includes fundamental aspects, various physical realizations (Spins, photons, atoms) as well as modern applications directly connected to research (e.g. quantum cryptography, entanglement, teleportation, quantum computation).

\section{References}

1. W. Dür, S. Heusler, Visualizing the invisible: The qubit as a key to quantum physics, Phys. Teach. 52, 489 (2014).

2. W. Dür, S. Heusler, What we can learn about quantum physics from a single qubit, arXiv:1312.1463 [physics. ed-ph] - http://arxiv.org/abs/1312.1463 


\author{
A Learning Path on Quantum Physics Including \\ Simulations, Low Cost Experiments, Online Resources \\ Massimiliano Malgieri, Pasquale Onorato and Anna De Ambrosis \\ University of Pavia, Italy
}

\begin{abstract}
Introduction and Theory
In the last two years we developed and tested a conceptual learning path on quantum physics for high school, based on Feynman's "sum over paths" approach. One of the distinguishing features of our educational path is the discussion of several modern experiments, from the 1980's on, which are used to highlight the main conceptual aspects of quantum physics.

Discussion of recent experiments in quantum optics combined with the adoption of the Feynman paths language allows to approach foundational themes from a modern point of view, and to provide a particularly clear formulation of the notoriously problematic concept of "wave-particle duality".

A well known difficulty in the teaching of modern physics is that in typical educational settings very little can be done from the experimental point of view. We try to overcome this difficulty by using a combination of several resources: low cost experiments, online resources and remote laboratories, and interactive simulation realized with the software GeoGebra.
\end{abstract}

\title{
Concept and Implementation
}

Our educational path has been developed around the discussion of the conceptual themes of quantum physics in relation to single photon interferometry experiments: two slits interference with one photon at a time, the Grangier experiment on photon indivisibility, Zhou - Wang - Mandel on the role of the acquisition of information on a quantum system, and others. To help students understand the structure of these experiments, we in some cases used GeoGebra simulations, while in others have adopted the interactive representations of experiments on the site QuantumLab of University Erlangen-Nuremberg. Compared to the first implementation, discussed at GIREP-MPTL 2014 in Palermo, the path has been enriched by a much wider discussion of typical stationary problem in one dimension from the point of view of Feynman's approach (particle in a hole and other bound systems, time-independent tunnel effect) and also by an experimental part, conducted with low cost materials. These include the measurement of $\mathrm{h}$ using LEDs of different colors, or the observation of spectral lines by a home-made spectrometer.

In the first year of experimentation, the path has been tested in training courses for high school teachers; while during the second year it has also been tested directly with students of the final year of high school.

\section{Objectives and Assessment}

The main aim of our work is to provide students with both a clear view of the conceptual and epistemological structure of quantum theory, and the tools for solving some of its typical problems. Our assessment of the achievement of this goal is based on a combination of data: Tests with open and multiple choice questions, traditional problem sheets, written argumentative papers produced by students, and final interviews. Results will be discussed in detail at the conference. 


\section{A Quantum Information Course with Python Simulations for Computer Science Students Sabine Tornow \\ Munich University of Applied Sciences, Germany}

\section{Introduction and Theory}

The concept of qubits, quantum computing and quantum information is a fascinating interdisciplinary field of physics, mathematics and computer science. The underlying basics of quantum mechanics are becoming more and more essential for students of engineering and computer science since today's information technology is increasingly governed by the laws of quantum mechanics. We present a quantum information course for students of computer science (bachelor) to be held at a university of applied sciences (Hochschule für angewandte Wissenschaften). The course is based on Python simulations and finite dimensional quantum mechanics. The only prerequisites are a basic knowledge of linear algebra, complex numbers, networks and programming - competencies which students of computer science after the third semester have generally acquired. The core of the lecture is built on Python simulation exercises covering different toy models and quantum algorithms. The Python code is highly readable, easily modifiable as well as interactive and so very useful for an inquirybased learning scheme.

\section{Concept and Implementation}

The duration of the lectures is 180 min per weeks (for 14 weeks), divided into classes and tutorials with hands-on training. Different Python programs are provided to the students as part of the tutorials and for the homework assignments, to support their own practicing. For communication outside class we are using Moodle.

The subjects covered during the semester are:

- short review of complex numbers, number theory, information theory and vector spaces (2 weeks),

- basic quantum concepts (2 weeks),

- qubits, quantum algorithm and cryptography (8 weeks),

- hardware, accompanied by a visit to one of the quantum computation labs in Munich (2 weeks).

\section{Objectives and Assessment}

With the knowledge of basic concepts - i.e., simple quantum systems, qubits, quantum cryptography, entanglement, the basic algorithms and possible hardware - the students should be able to assess and be part of computational scientists' developments of new technologies where quantum effects play key roles.

The core of this course is represented by the Python codes of selected model systems and by the simulation of the respective quantum algorithms. This leads to inquiry-based learning since not only visualization and animations but in particular the direct manipulation of the code fosters a deeper understanding of this challenging but intriguing subject.

Achievement of the learning objectives as well as of the lecturing itself is evaluated by formative anonymous concept tests during the semester. At the end of the semester the students will attend an oral examination covering the course contents and present a term paper on a proposed or self-chosen project. 


\author{
An Interactive On-line Course in Contemporary Physics \\ Dean Zollman ${ }^{1}$, Raiya Ebini ${ }^{1}$ and Ulaş Üstün ${ }^{1,2}$ \\ ${ }^{1}$ Kansas State University, USA, ${ }^{2}$ Artvin Çoruh University, Turkey
}

\begin{abstract}
Introduction and Theory
A typical model for an on-line course, particularly for a MOOC, is a collection of lectures on video. Frequently, the lectures are divided into short segments which are one-way transmission. Thus, they do not take advantage of physics education research which shows that students learn better when they are actively involved.

Kansas State University has offered a face-to-face course on quantum physics for students who are not majoring in physics. This course, Contemporary Physics, includes hands-on activities, computer visualizations, concepts and very little mathematics. Overall, the course was successful in terms of learning but attracted only about 20 students per semester.
\end{abstract}

\title{
Concept, Objectives and Implementation
}

To reach a large audience and to experiment with interactivity within an on-line environment we decided to create Contemporary Physics On-Line. Our primary goal was to create a learning environment which would include a high level of interactivity while students learn about quantum mechanics and its applications. As with the face-to-face course our audience is students who are not studying physics at the university.

The core of the course is Visual Quantum Mechanics (https://web.phys.ksu.edu/vqm/). This set of learning modules was developed for interactively teaching concepts of quantum mechanics to secondary school students. It has been used successfully in a variety of secondary and university settings. The original materials rely heavily on worksheets in which students read introductions, conduct activities - both hands-on and simulations - and respond in writing to questions. Teachers lead discussions after the questions have been answered. The core materials were supplemented visualizations from the PhET project and YouTube videos. To assure interactivity we included the questions and responses as on-line "quizzes" and made available chat rooms where students could discuss the materials. The most recent version of the course is available at https://k-state.instructure.com/courses/3249.

\section{Assessment}

The course has been offered twice to a total off five students. Learning was assessed by administering the same examinations that were used in recent offerings of the face-to-face course. The results have been mixed. Feedback on the structure of the course and the interactivity has been collected from most of the students. The comments have varied and seem to be correlated to the level of success in learning the content. As a result of these pilot studies we will be modifying the course and offering it to a broader audience in 2016.

\section{Acknowledgements}

The development of the course is supported by the KSU Global Campus. Dr. Üstün's participation is supported by The Scientific and Technological Research Council of Turkey (TÜBİTAK). 
PARALLEL SESSION VRL/MAP:

\title{
VIRTUAL AND REMOTE LABS IN PRACTICE
}

Automated Experiments for EJsS Laboratories

Daniel Galan ${ }^{1}$, Ruben Heradio ${ }^{1}$, Luis de la Torre ${ }^{1}$, Sebastian Dormido ${ }^{1}$ and Francisco Esquembre ${ }^{2}$ ${ }^{1}$ National Distance Education University (UNED), Spain, ${ }^{2}$ University of Murcia, Spain

\author{
A Solar Cell Virtual Laboratory \\ Jacobo Sáenz, Luis de la Torre and Sebastián Dormido \\ UNED, Spain
An Evaluation of a Cross National Transfer of an Online Laboratory Activity on the Optics of the Human Eye
Elliot Mylott ${ }^{1}$, Heike Theyßen ${ }^{2}$, Sarah Struzyna ${ }^{2}$, Ralf Widenhorn ${ }^{1}$
${ }^{1}$ Portland State University, USA, ${ }^{2}$ University of Duisburg-Essen, Germany

\section{CONCEPT MAPPING}

Physics Teacher Candidates' Views About Technology-Assisted Concept Mapping Method (TACM) and Difficulties Encountered in the Preparation of TACM in Different Languages

Özlem Oktay ${ }^{1}$ and Ahmet Illhan Şen ${ }^{2}$

${ }^{1}$ Middle East Technical University, Turkey, ${ }^{2}$ Hacettepe University, Turkey 


\author{
Automated Experiments for EJsS Laboratories \\ Daniel Galan ${ }^{1}$, Ruben Heradio ${ }^{1}$, Luis de la Torre ${ }^{1}$, Sebastian Dormido ${ }^{1}$ and Francisco Esquembre ${ }^{2}$ \\ ${ }^{1}$ National Distance Education University (UNED), Spain, ${ }^{2}$ University of Murcia, Spain
}

\title{
Introduction and Theory
}

Existing software allows the development of high quality interactive applications that permit their implementation in laboratories, both virtual and remote. To perform an experiment, the user needs to know how these applications are implemented. In addition, when coding an experiment, the user has to manage the language in which the simulation was created and needs to handle fluently a simulation language just to perform experiments. In the academic field these simulations are used to create virtual and remote laboratories. The authors propose a tool to develop automated experiments for these laboratories. It is intended that the experiments use a powerful and simple language to improve students interaction with the laboratories. That is, avoid repetitive tasks using simple algorithms, data collection and analysis of results, among other features.

\section{Concept and Implementation}

In this paper we propose a new environment for the development of experiments in the authoring tool Easy java(script) Simulations (EjsS). It permits the decoupling of the application design phase from the experiment design. It has been equipped with a new language capable of encompassing all the desired commands, such as creating events, launching simultaneous applications or modifying their variables. Set with a high level layer, the language allows less experienced users to create their own experiments without having any knowledge of how the application was made. It maintains a balance between simplicity and functionality. These features will be explained throughout the article and some physic and control examples of use will be given to demonstrate the potential of this new environment.

\section{Objectives and Assessment}

The main objective of this work is to create an operational and useful tool for students that allows conducting experiments in virtual and remote laboratories of the university.

The experiment editor described in this paper has been implemented at UNED and it has passed a testing phase by some students and members of the university. Automatic control students have mandatory laboratory sessions. In the laboratory session, half of the students used the Experiment Editor and the other half no. The analytical evaluation of this experience shown that the use of the experiment editor assists students in performing the proposal experiences and improving their final grades. 


\author{
A Solar Cell Virtual Laboratory \\ Jacobo Sáenz, Luis de la Torre and Sebastián Dormido \\ UNED, Spain
}

\begin{abstract}
Introduction and Theory
Actually virtual and remote laboratories plays an important role in the students learning process. For distance education in scientific areas, where the laboratories are an essential part, this importance is enhanced.

This work presents an online virtual laboratory of solar cells. The simulation allows students for studying the behavior of this system by changing parameters. By this method, the users obtain estimations of the outputs for a wide range of states and prepare themselves for the remote version of the laboratory, with real equipment.
\end{abstract}

\title{
Concept and Implementation
}

The virtual lab is created using the open source tool EJsS (Easy Java/Javascript Simulations). This tool is used for creating of all kind of simulations, by introducing the equations of the system, and interact with the model using a graphical user interface. The laboratory provides to the user a collection of sliders and fields to modify parameters and obtain the characteristic curve. Some of these fields, that the student can change are environmental variables as irradiance and temperature, configuration parameters like the number of serial and parallel cells in the array. Also the user can modify geographic and time-related parameters like latitude, longitude, date, local hour and orientation.

Additionally, the numerical and graphical data obtained during the simulation can be stored easily in .m files for later analysis with mathematical software as Matlab.

\section{Objectives and Assessment}

The work presents one virtual lab to improve the students knowledge about configuration and performance of solar cells. On the one hand, this single simulation contains different tabs to develop at least eight different experiences (i.e. laboratory practices). On the other hand, the remote version of this laboratory is now in development and complement the theoretical results of the virtual one.

The results in this kind of laboratories show that the computer based simulations offer the student different practices without the physical devices needed. But until the completion of the remote version we have no information about the impact in the students learning. Then this lab offers similar ways of work to their traditional counterparts and some additional advantages, like the fast-to-change parameters and the possibility of been the previous step to the remote laboratory. 


\author{
An Evaluation of a Cross National Transfer of an Online Laboratory Activity \\ on the Optics of the Human Eye \\ Elliot Mylott ${ }^{1}$, Heike Theyßen ${ }^{2}$, Sarah Struzyna ${ }^{2}$, Ralf Widenhorn ${ }^{1}$ \\ ${ }^{1}$ Portland State University, USA, ${ }^{2}$ University of Duisburg-Essen, Germany
}

\title{
Introduction
}

The increase in digital media in physics education has made the sharing of online laboratory activities easier than ever before. Despite the ease with which educational platforms can be shared between institutions in different countries, a number of questions addressing the efficacy of such transfers remain. These issues include the necessity of translation as well as differences in the participating countries' educational systems and student populations. In this talk we present the findings of a study of the transfer of an online laboratory activity from a university in Germany to a university in the USA.

\section{Implementation}

The universities in this study offer general physics within the context of biology and medicine, which is intended for pre-health and medical students. The transferred online laboratory activity was developed in Germany at Heinrich-Heine University of Düsseldorf (HHU) and has been used in general physics courses for medical students for over a decade. The interactive laboratory activity is divided into three sections: geometrical optics, lenses, and the optics of the human eye. The content is presented online and the hands-on experiments are replaced by Interactive Screen Experiments (ISE). The content was translated into English and transferred to Portland State University (PSU) in the USA. It was also presented to students at the University of Duisburg-Essen (UDE) in Germany. Neither PSU nor UDE had offered students the online lab activity previously.

\section{Assessment}

Students were surveyed before the study for information about their personal background, selected cognitive abilities, and physics self-concept, all of which have been shown to be predictors for learning. Students' previous content knowledge and conceptual understanding of the activity's learning goals were assessed through pre and post quizzes. A questionnaire was also given to students after completing the activity exploring students' use of and attitudes toward the activity. Because the online lab activity was already in use at HHU, assessment data concerning learning gains and attitudes was only compared between PSU and UDE.

We found that students from PSU and UDE differed with regard to age, self-concept, and previous knowledge, which is likely due to the differing educational systems. Both the UDE and PSU cohorts showed a large gain in conceptual understanding. PSU students showed an even higher gain, which could be due to multiple factors including the amount of time spent on the lab activity or the higher previous knowledge of the UDE students. Students' attitudes toward the activity were positive, similar between groups, and were correlated with students' physics related self-concept. Because of the positive learning gains and attitudes, we conclude that the transfer of the lab was successful. 


\section{Physics Teacher Candidates' Views About Technology-Assisted Concept Mapping Method (TACM) and Difficulties Encountered in the Preparation of TACM in Different Languages Özlem Oktay and Ahmet IIhan Şen ${ }^{2}$ \\ ${ }^{1}$ Middle East Technical University, Turkey, ${ }^{2}$ Hacettepe University, Turkey}

Concept mapping (CM) which was developed on the theory of Ausubel's meaningful learning has been widely used in education. It has changed and developed over time accepted as teaching and assessment tools in all levels of teaching. Initially implemented by paper and pencil form, this method can be prepared with electronic or online format by the means of technology. In this way, prepared concept maps undoubtedly provide important advantages in concept adding, deleting or changing in a technological environment. It also creates a more affective and motivating teaching and learning atmosphere. However, there is limited research combined with technology and CM at the same time. In addition, when CM is used, it is important to consider the structure of the language. Generally, learners have difficulties to select connection words and link them in a meaningful manner with the concepts. With the scope of this study we examine the following research questions:

1. What are the physics teacher candidates' views about technology-assisted concept mapping method (TACM)?

2. What are the difficulties students encounter when preparing CM in different languages? For this aim, data were collected from physics teacher candidates participated in method courses in which they learned CM applications. Participants came from two different universities in which the languages of instruction are English and German respectively. A questionnaire was developed by the researchers and implemented to teacher candidates. Participants were asked to prepare CM with paper-pencil and technological tools (Cmap). After that, both applications were compared in order to identify teacher candidates' difficulties. Data consisted of materials from teachers and open-ended questions. Qualitative analysis technique was used to prepare coding themes. Two researchers independently evaluated the CM materials and discussed together to achieve an agreement on its quality. Based on the study results, teacher candidates had difficulties when they prepared CM with technology. Fear on the use of technology, lacking of technological skills and experiences with CM software program were the most important obstacles during the implementation. It was also observed that participants easily prepared CM materials in English and German languages, on the other hand they had considerable problems with respect to Turkish. These results may be helpful to reflect real situations of CM methods and software applications in the classrooms. 


\title{
WORKSHOP CAM:
}

\author{
The Amazing World of Ionizing Particles: \\ Experiments With MX-10 Particle Camera \\ Peter Žilavý1, Zdeňka Koupilová1, Vladimír Vícha ${ }^{2}$ and Vladimír Stanislav ${ }^{3}$ \\ ${ }^{1}$ Charles University in Prague, Czech Republic, \\ ${ }^{2}$ Czech Technical University in Prague, Czech Republic, \\ ${ }^{3}$ Jablotron Alarms a. s., Czech Republic
}

\begin{abstract}
Introduction and Theory
Particle camera MX-10 is a unique educational device capable of detecting and displaying ionizing radiation. It can visualize radiation similarly to a cloud chamber.

Using this device we can demonstrate phenomena in a way which is impossible with traditional school detectors and devices (such as GM tubes). Powered by the Timepix chip created in CERN within the Medipix2 collaboration and using the Pixelman control software (developed by the Institute of Experimental and Applied Physics of the Czech Technical University in Prague), the MX-10 is a useful tool for bringing live particle physics right into school or university lab.
\end{abstract}

\section{Concept and Implementation}

The workshop presents a series of school experiments with the MX-10 particle camera demonstrating the elementary phenomena connected to ionizing radiation like existence of radiation from natural or artificial sources (including objects around us - potash fertilizer, uranium glass and other), some properties of alpha, beta and gamma radiation, radiation shielding by various materials (paper, food wrapping film, different metal plates, water and other materials).

Objectives and Assessment

At the workshop, participants will have the opportunity to try out the device and carry out several experiments that illustrate selected properties of ionizing radiation. These experiments use a set of safe and generally available natural and artificial radiation sources like uranium glass, potash fertilizer, thoriated tungsten welding rods, safe school source of alpha particles (americium 241Am) and other.

Information about the MX-10 particle camera and experiments are available at websites: http://www.particlecamera.com http://www.particlecamera.com/index.php/support-camera-mx-10/experiments-with-mx-10 


\title{
INVITED SYMPOSIUM iMP:
}

\author{
IMOBILEPHYSICS (IMP): \\ USING SMARTPHONE, TABLET-PC \& CO. AS EXPERIMENTAL TOOLS \\ Jochen Kuhn (Chair), University of Kaiserslautern, Germany \\ Andreas Müller (Discussant), University of Geneva, Switzerland
}

Mobile devices, especially smartphone and tablet-PC, have increasingly become everyday tools, particularly for the younger generation. In recent years, various contributions have been published about the use of smartphones and tablet-PCs as experimental tools especially in physics classroom and undergraduate studies. This is possible because today's smartphones and tablet-PCs are equipped with many sensors, which can be used to perform quantitative measurements of sound, acceleration, radioactivity, light intensity, position on earth (GPS) etc. In this symposium different examples of using these mobile devices as experimental tools in classroom and undergraduate studies will be presented and discussed.

iMobilePhysics:

Possibilities and Limits of Using Smartphone and Tablet-PC as Experimental Tools Jochen Kuhn ${ }^{1}$ and Andreas Müller ${ }^{2}$

${ }^{1}$ University of Kaiserslautern, Germany, ${ }^{2}$ University of Geneva, Switzerland

iAcoustics:

Study Acoustic Phenomena with Smartphone and Tablet-PC as Experimental Tools Michael Hirth ${ }^{1}$, Jochen Kuhn ${ }^{1}$ and Andreas Müller ${ }^{2}$

${ }^{1}$ University of Kaisers/autern, Germany, ${ }^{2}$ University of Geneva, Switzerland

\author{
Physics in Your Pocket: Experimenting and Learning with Your Smartphone \\ Manuel Á. González and Miguel Ángel González \\ University of Valladolid, Spain
}

\section{Multiple Smartphones Interests as Educational Tools in Digital Active Classes Philippe Jeanjacquot and Françoise Morel-Deville ACCES- Institut Français de l'Education - Ecole Normale Supérieure, France}

\author{
Smartphones as Pocket Labs for DIY Experimental Physics \\ Joël Chevrier \\ CIME Nanotech, Université Joseph Fourier and Grenoble INP, France
}

physics.move: Teaching Experimental Physics by Using Mobile Motion Analysis

Pascal Klein ${ }^{1}$, Sebastian Gröber ${ }^{1}$, Jochen Kuhn ${ }^{1}$ and Andreas Müller ${ }^{2}$

${ }^{1}$ University of Kaiserslautern, Germany, ${ }^{2}$ University of Geneva, Switzerland 


\author{
iMobilePhysics: \\ Possibilities and Limits of Using Smartphone and Tablet-PC as Experimental Tools \\ Jochen Kuhn ${ }^{1}$ and Andreas Müller ${ }^{2}$ \\ ${ }^{1}$ University of Kaiserslautern, Germany, ${ }^{2}$ University of Geneva, Switzerland
}

\title{
Introduction and Theory
}

iMobilePhysics (iMP) deals with using internal sensors of everyday modern communication technology as experimental tools. Examples of experiments and their integration into classroom physics have already been presented.

We expect the motivation as well as the learning outcome to be increased by using smartphones as experimental tools, compared to traditional physics class with experiments of the same content. This is based first on the theoretical framework of context based learning, after which the connection of an experimental tool to everyday life has a positive influence on motivation. In this aspect, self-efficacy as one important component of motivation is of special interest and of particular importance to situated and context-based learning in general by offering an opportunity of participation. Furthermore it's well known that competent handling of multiple representations is supposed to be significant for learning and problem solving - especially for conceptual learning in science education. With those multimedia tools, experimental data are dynamically represented in different formats, such as diagrams, tables, etc. Translation between the real-world experiment and measured data is a key competence of physics and can be fostered by the possibilities those multimedia tools offer. Besides the importance of multiple representations for better learning, it's presumed to be confirmed that curiosity is one of the three pillars for academic performance, too.

\section{Concept and Implementation}

The contribution discuss the possibilities and the limitations of the iMP-concept by presenting two quasi-experimental, intervention-control-group studies in high school physics: one study in Acoustics ( $10^{\text {th }}$ grade; $N_{\text {total }}=58, N_{\mathrm{IG}}=28 ; N_{\mathrm{CG}}=30 ; 49 \%$ female, $51 \%$ male; average age $=15.3$ ) and one study in Mechanics (senior class; $N_{\text {total }}=154 ; N_{\mathrm{IG}}=87, N_{\mathrm{CG}}=67 ; 51 \%$ female, $49 \%$ male, average age $=16.7$ ). While students in the intervention group conducted experiments using mobile devices as experimental tool, the students in the control group examined the same contents with traditional experimental tools. The study was imbedded into regular physics classes and conducted as pre-postfollow up design.

\section{Objectives and Assessment}

Based on the theoretical and conceptual framework mentioned above we study the following hypotheses:

1. Using everyday devices as experimental tools will foster conceptual understanding and motivation.

2. Especially the subscale "self-efficacy" as one of the important motivational pillar for context-based learning will be augmented.

3. Using everyday devices as experimental tools will foster curiosity concerning analyzing physics phenomena.

We study these dependent variables by using well-established and well-validated paper-andpencil tests. The results showed that in the framework of acoustics self-efficacy as well as conceptual understanding could be fostered significantly and with middle up to large effect sizes compared to the control-group. Furthermore students' curiosity in the topic mechanics is significantly higher in the intervention group compared to their counterparts in the control group after the treatment.

Although the results of the studies are encouraging, more work has to be done with larger sample size and in different topics. 


\author{
iAcoustics: \\ Study Acoustic Phenomena with Smartphone and Tablet-PC as Experimental Tools \\ Michael Hirth ${ }^{1}$, Jochen Kuhn ${ }^{1}$ and Andreas Müller ${ }^{2}$ \\ ${ }^{1}$ University of Kaiserslautern, Germany, ${ }^{2}$ University of Geneva, Switzerland
}

\begin{abstract}
Introduction and Theory
Internal sensors in smartphones and the availability of apps enable us to perform manifold measurements with sufficient accuracy. Most smartphones include a microphone, an acceleration sensor, a pressure transducer, a GPS receiver, a camera or even more sensors. Many traditional computer-assisted experiments could also be conducted with these devices, but with less experimental effort. Consider the subject field of acoustics for example: Speaker and microphones can generate and detect sound in a range between $[20 \mathrm{~Hz}, 24 \mathrm{kHz}]$ and apps display acoustic data in real time.

From an educational perspective smartphones are intuitive experimental tools with high motivational potential. Special attention should be paid to the unique characteristics of smartphones as mobile measuring tools. Physical data can be executed at almost any location and time making the application of these tools open for out-of-classroom experiences. With the intention to increase opportunities for autonomous experimentation, smartphones can be used to gather data either in formal homework or even in informal learning settings.
\end{abstract}

\title{
Concept and Implementation
}

The iPhysicsLab is a special school lab at the University of Kaiserslautern, founded in 2013. There students from German grammar schools are engaged to experiment with smartphones in different disciplines, e.g. mechanics and acoustics. The presentation focusses on a single acoustic experiment which is undertaken by upper secondary school students. It illustrates the different aspects of studying physics with smartphones

- the measurement accuracy,

- straightforward implementation of smartphones as experimental tools into a formal learning setting

- problems arising by using applications (usability, bugs)

- and in a further investigation an out-of-classroom application of smartphones.

\section{Objectives and Assessment}

A short acoustic pulse is emitted into a both open ended tube. A mobile device detects the primary and a sequence of secondary pulses in an oscillogram. The latter are a result of manifold reflections of the pulses on the open ends. The experimental setup provides a) an accurate method to determine the speed of sound in air b) a motivation to discuss about the end correction of open ended tubes c) an opportunity to observe phase shifts of acoustic pressure waves when the reflections occur on open ends. The physical principles of this experiment can be transferred to a common real life phenomenon: When a train is running through a tunnel, passengers often complain about discomfort, which is caused by a feeling of changing pressure on the ears. Pressure measurements within the train support that the origin of this phenomenon are a compression wave and a depression wave, which are generated when the train's nose and rear enters the tunnel's entrance. The pulses propagate with the speed of sound to the open ends and are reflected with a phase shift of the pressure for several times similar to the acoustic pulses in the tube described above. 


\section{Physics in Your Pocket: Experimenting and Learning With Your Smartphone \\ Manuel Á. González and Miguel Ángel González \\ University of Valladolid, Spain}

\section{Introduction and Theory}

Along the last years the use of mobile devices in education has increased hugely. This increase includes not only the use of ICTs as learning facilitators. Mobile devices have also become useful tools in experimental physics thanks to their rich sets of built-in sensors[1].

\section{Concept and Implementation}

The use of smartphones as measurement devices in physics experiments requires careful attention to ensure good learning outcomes. Some aspects that must be considered are the reliability and accuracy of the smartphone sensors as well as their adequacy to the experiment. Also, the precision and correctness of the applications used to access the sensors data are essential to obtain reliable results[2]. Another important aspect of this technique is designing interesting experiments that can be done with the smartphones allowing the students to observe the physical phenomena without technical or theoretical difficulties. Finally, the influence of these experiments on the students learning must be carefully analyzed. Some of these considerations will also be discussed in other contributions to this same symposium[3].

\section{Objectives and Assessment}

We pretend to improve physics learning, both by making theoretical concepts easily available to the students and by allowing the students to observe the nature and experiment by themselves, confronting their own experimental results with their beliefs or previous knowledge. Along this second aim, the use of students' smartphones allow them to experiment both in the laboratory or in many everyday activities. The use of smartphones as measuring devices in the teaching laboratories will also permit to substitute, under certain conditions, expensive laboratory devices by the more versatile smartphones. These 'low-cost' laboratories would benefit institutions with large number of students and small budgets.

Currently we work with high school students and undergraduate university students, what allows us to observe the difficulties and results of different types of students. In order to analyze the influence of smartphones as experimental tools on students' learning we perform qualitative and quantitative analysis. For the qualitative study, students are surveyed to obtain feedback on their work with the applications and the smartphones. For the quantitative analysis, the students' grades and engagement are measured and analyzed.

\section{References}

1. Kuhn, J., \& Vogt, P. (2013). Applications and examples of experiments with mobile phones and smartphones in physics lessons. Frontiers in Sensors, 1 (4), 67-73.

2. González, M. Á., González, M. Á., Martín, M. E., Llamas, C., Martínez, Ó., Vegas, J., Herguedas, M., \& Hernández, C. (2015). Teaching and Learning Physics with Smartphones. Journal of Cases on Information Technology (JCIT), 17(1), 31-50.

3. Kuhn, J. iMobilePhyisics: Possibilities and Limits. This symposium 


\section{Multiple Smartphones Interests as Educational Tools in Digital Active Classes Philippe Jeanjacquot and Françoise Morel-Deville ACCES- Institut Français de l'Education - Ecole Normale Supérieure, France}

\section{Introduction and Theory}

Increasing the opportunities of access to knowledge in the classroom, home and social network of students, mobile connected devices are changing the ecology of the teaching and learning models: student-teacher interactions, spaces at school and during private time and deregulation of active cross disciplines.

A team of 25 teachers in middle and high school, in physics, biology, geology, maths, languages, geography, philosophy and economy works on this subject. Exploring new opportunities of teaching, with the Smartphones (and every kind of mobile devices), their sensors, their connectivity, their mobility or their computing power. The goals of this team are:

- to design and carry out activities with mobile devices.

- to observe the activities of their peers

- to analyse the benefits and the limits of the use of mobile devices during their activities

- to share their exploration to the community thanks to a website and teachers training. (http://acces.ens-lyon.fr/acces/classe/numerique)

\section{Concept and Implementation}

Our Institute (IFé) French Institute for Education, is the national research center for education. Different teams of researchers work with associated teachers. Our team follows this method to work on the impact of the use of the mobile devices for teaching. The team members are teachers and also researchers in the field of education. The members work together on different subjects:

- the uses of the sensors for experimental sciences.

- the new way to teach with mobile devices. What shall we change when the students are connected. The use of new media for keeping notes of the courses. The new way to manage the class with the use of mobile devices

- The part of the mobile devices in the flipped or hybrid classroom.

But also the legal way to use the smartphones of the students in our activities (most of the schools do not allow the use of mobile phone)

\section{Objectives and Assessment}

The objective is to check the impact of the use of the mobile devices on the pupils, teachers, school and parents

- Do the pupils learn better with their mobile devices?

- Do they have a new sight on their device: it is not only for chatting or gaming but also for learning?

- The activities are more efficient and pleasant for the teacher?

- Is it easy for the administrative staff to manage the classes with mobile devices?

- How the parents fell this new way of teaching (we shall inform them)?

Every month a meeting allows us to assess or explorations. We are partner with the center of educational experiments (the CARDIE in French), this is a department of the ministry of education which allow, follow, advise and assess the experimental teaching. Their advisers have an external sight of the project and help us to prepare our assessment. 


\section{Smartphones as Pocket Labs for DIY Experimental Physics Joël Chevrier \\ CIME Nanotech, Université Joseph Fourier and Grenoble INP, France}

\section{Introduction and Theory}

There are about 2 billion smartphones in the world. This is not only an avenue to teach experimental physics, but equally important, it provides new pedagogical possibilities. There are up to 10 sensors in a single smartphone, all real-time connected to digital world. Among them, 3D accelerometer and 3D gyroscope enable physics students to do all key experiments in Classical Mechanics, a course taught in all universities in the world. The sensor performances are so high that quantitative experiments can be done in a "frugal environment": pendulum experiment with detailed numerical analysis of data is done by suspending the smartphone to its own charge cable held in hand. For all experiments, no more specific apparatus (maybe a pillow on the floor...) is needed than a smartphone to collect data and a PC to treat them either in real-time or after experiment.

\section{Concept and Implementation}

Following pioneering works ${ }^{1}$, we have identified and developed 5 basic sessions related to major pedagogical objectives in Classical Mechanics: 1D movements (free fall and elevator), rotation (turn table or salad spinner), pendulum, oscillations (using a rubber elastic) and study of tramway trajectory. The use of smartphones enables a real time and interactive representation of collected data on laptops. For this, we have developed a free software iMecaProf that can be used with iPhone and Android smartphones. For a circular motion, as the smartphone rotates, its orientation is represented real-time on a PC screen together with acceleration and velocity vectors in lab and smartphone frames (using polar coordinates) and with energies versus time (potential, kinetic and mechanical energies). The taught formal description becomes an interactive representation used in real time to even control by hand the experiment. Students finally explore direct connections between Classical Mechanics and daily life: smartphones use sensors whose data are analyzed within the frame of Classical Mechanics for their every day uses.

\section{Objectives and Assessment}

Based on rich possibilities offered by smartphone to teach physics, the pedagogical contents is always evolving: i) interactive and real-time representation of data based on formal description ii) quantitative numerical treatment after data collection, iii) student innovation especially when defining frugal experimental environments, iv) impact of sensor performances and limits on results.

A program has been developed on this basis for first year students in sciences at the University of Grenoble. Last year, this experimental teaching with smartphones involved 25 students during 20 hours and this year, 50 students. We have not yet entered a careful assessment beyond student engagement and apparent satisfaction. It is a next step to find a collaboration to do it.

\section{References}

1. J.Kuhn and P.Vogt, Column Editors of iPhysicsLabs in The Physics Teacher: https://www.aapt.org/ Resources/iPhysics-Labs.cfm 


\author{
physics.move: Teaching Experimental Physics by Using Mobile Motion Analysis \\ Pascal Klein ${ }^{1}$, Sebastian Gröber ${ }^{1}$, Jochen Kuhn ${ }^{1}$ and Andreas Müller ${ }^{2}$ \\ ${ }^{1}$ University of Kaiserslautern, Germany, ${ }^{2}$ University of Geneva, Switzerland
}

\begin{abstract}
Introduction and Theory
Drop-out rates of introductory physics courses at German universities are about $30 \%$. Students explain the difficulty and the complexity of the learning content as well as decreasing motivation to be main reasons for this trend.

physics.move addresses this study entry phase with experimental activities: In the setting of weekly recitations, students will adapt their theoretical knowledge to application-oriented problems. Traditional paper-and-pencil based exercises are extended by video-based experiments which can be analyzed quantitatively (so-called Video Motion Analysis tasks). This allows a deeper connection between theory and experimentation. After students gained first experiences with VMA exercises, they conduct hands-on experiments themselves, record them and analyze them with mobile devices (mVMA task).

Within the scope of physics.move, we (1) design instructions referring to such video-experiments, (2) investigate the feasibility of our approach for large lecture courses $(\mathrm{N}>50)$, (3) investigate students' learning and motivation with standardized instrument with intervention- and control-groups and (4) develop and validate new assessment instruments (focusing on representation competency and authenticity).
\end{abstract}

\title{
Concept and Implementation
}

In this contribution we report the results of a quasi-experimental, intervention-controlgroup studies concerning undergraduate physics courses (Ntotal $=168$ ).

\section{Objectives and Assessment}

Based on the theoretical and conceptual framework mentioned above we study hypotheses and research questions concerning workload, perceived stress, motivation, epistemic curiosity, conceptual understanding of mechanics and representation competency.

For that purpose, both groups, IG and CG, were identical in their learning contents and differed only in the use of mobile devices as experimental tools in the intervention group. We examined the dependent variables by using well-established and well-validated paper-andpencil tests and controlled for prior knowledge, instructor behavior and time-on-task.

First results show that self-efficacy as a component of motivation as well as conceptual understanding could be fostered significantly and with middle up to large effect sizes compared to the control-group. Furthermore the curiosity of the undergraduate students in the intervention group is significantly higher compared to their counterparts in the control group after the treatment. 


\title{
INVITED SYMPOSIUM GBL:
}

\author{
GAME-BASED LEARNING IN PHYSICS AND PHYSICS EDUCATION \\ André Bresges (Chairperson), University of Cologne, Germany \\ Michael A. Dubson (Discussant), University of Colorado Boulder, USA
}

Digital games use motivational methods to keep players immersed in the gaming environment for a prolonged time, but educators often struggle to apply gaming effectively in their classes. Game Based Learning (GBL) is not constrained to digital environments. Role playing, constructing models, some forms of cooperative learning, and the visualization of actual scientific data and technology (i.e. CERN's Large Hadron Collider, flight simulations) are another form of gaming, and may be blended with a digital environment to provide additional layers of depth and a diversity of methods for in-classroom use.

This symposium focuses on case studies that demonstrate the diversity of GBL Methods.

The Role of Metacognitions in Serious Gaming

Jörg Zumbach, Stephanie Moser, Ines Deibl, Alexander Strahl

University of Salzburg, Austria

\author{
Evaluating the Use of Flight Simulators \\ for the NASA/AAPT "Aeronautics for Introductory Physics" Educator Guide \\ Rebecca Vieyra ${ }^{1}$, Florian Genz $z^{2}$ and André Bresges ${ }^{2}$ \\ ${ }^{1}$ NASA Aeronautics Research Mission Directorate, USA, \\ ${ }^{2}$ University of Cologne, Germany
}

\begin{abstract}
CERN's Media Lab Games in Upper Secondary Physics Education: Overcoming Baek's Barrier to uptake Computer Games in Classrooms

Oliver Keller ${ }^{1}$, André Bresges ${ }^{2}$ and Nadine Westkamp ${ }^{2}$

${ }^{1}$ CERN Media Lab, Switzerland, ${ }^{2}$ University of Cologne, Germany
\end{abstract}

\author{
Gaming, Visualization and Sharing Data \\ Initialize Learning in Educational Activities at CERN \\ Konrad Jende, Rolf Landua \\ CERN, Geneva, Switzerland
}

\section{Game-Based Learning for Supporting Self-Confidence and Motivation of Female Physics Students \\ Bernhard Ertl \\ Donau-Universität Krems, Austria}

\author{
Exploring Physics with Video Games \\ Aaron Titus ${ }^{1}$ and Francisco Esquembre ${ }^{2}$ \\ ${ }^{1}$ High Point University, USA, ${ }^{2}$ Universidad de Murcia, Spain
}




\author{
The Role of Metacognitions in Serious Gaming \\ Jörg Zumbach, Stephanie Moser, Ines Deibl, Alexander Strahl \\ University of Salzburg, Austria
}

\title{
Introduction and Theory
}

Game-based Learning is an educational method that is meanwhile widely used by educators within different fields. Also in Physics education the use of games for introducing and deepening physical concepts has become more and more popular. Nevertheless, game-based learning includes a variety of approaches that differ in content, the character of entertainment and, thus, sometimes in their mission. The question that arises here is whether such games are designed rather for entertainment purposes (edutainment) or as a vehicle for supporting the construction of appropriate physical knowledge within a domain. Following the latter one, the term of serious games is used within the context of this research. The basic idea beyond here is the use of game elements that have entertainment character only as a secondary objective. The main objective still remains on the educational purpose of such application. Thus, the cognitive processing of such learning resources is central in order to ensure sustainable knowledge acquisition. A key factor for supporting knowledge acquisition from multimedia learning material, simulations or serious games is known as metacognition. Metacognitions, either as learner characteristic, trained or scaffolded within learning environments can significantly contribute to the success of self-directed learning.

\section{Concept and Implementation}

Within this research, a single game about the conservation principle has been used within an experimental study. The game which is also a simulation is about a skateboarder within a half pipe (University of Colorado Boulder, 2015). The interface offers several possibilities of interaction and external visualization of different variables. Within a $2 \times 2$ factorial design the role of metacognitive training prior to use of the game (with or without) and the role of metacognitive prompting (with or without) is examined using a pre- and post-test related to knowledge acquisition, metacognitive behavior, cognitive load and mental effort.

\section{Objectives and Assessment}

The objective of this research is to analyze the impact of metacognition during serious gaming using the simulation as described above. The hypotheses to be tested are that learners with training in metacognitive strategies would perform better than those without such training in knowledge post-test and would also report about a higher mental effort. We also assume that the prompting of metacognitive behavior would improve the learning outcomes, but only within learners with training of metacognitive behavior. Participants are 8th graders in Austrian High Schools and are randomly assigned to one of the four conditions. The presentation will present the basic findings as well as limitations of this experiment. 
Invited Symposium GBL

Room B (D Z005)

Contribution 4B2

Thu, 10 Sep, 14:20-14:35

\author{
Evaluating the Use of Flight Simulators \\ for the NASA/AAPT “Aeronautics for Introductory Physics" Educator Guide \\ Rebecca Vieyra ${ }^{1}$, Florian Genz ${ }^{2}$ and André Bresges ${ }^{2}$ \\ ${ }^{1}$ NASA Aeronautics Research Mission Directorate, USA, \\ ${ }^{2}$ University of Cologne, Germany
}

\begin{abstract}
Introduction and Theory
Airplanes are fascinating for students, and the question "why airplanes fly" is frequently asked of teachers and parents accordingly. However, neither U.S. nor European textbooks cover the topic in depth. Many textbooks and educational resources provide oversimplified or even erroneous explanations that can lead to significant misconceptions about flight and fluid dynamics. The poor state of explanations of flight physics in textbooks has several reasons: working on solutions of the Navier-Stokes Equation in classroom used to be impossible, and the computing power that is necessary to use simulations has historically been out of reach for classroom scenarios. Thus, over-simplifications dominating physics textbooks often make disputable use of Bernoulli's Law. Furthermore, one cannot explore the context by real flight experience with a class of students.

Flight simulator games, on the other hand, provide the necessary computing power and may serve as a context that is easy to exploit in classroom.
\end{abstract}

\title{
Concept and Implementation
}

Founding on NASA's and the American Association of Physics Teachers' joint project "Aeronautics for Introductory Physics" educator guide (Vieyra, Genz et al, 2015, NASA publication \#EG-2015-03-005-HQ), we develop a laboratory course using a classroom wind tunnel (Leybold 37306) and a commercial off-the-shelf flight simulator game (X-Plane 10). The concept is based partly upon science education research done through the Educational Gaming Environments (EdGE) Project at TERC (http://edgeatterc.com) suggesting that casual games bridged with in-class learning results in greater student gains than in those students asked to play outside of class without directly connecting it to the course content (Rowe et. al., 2014). Using a simple shortcut (Ctrl-M), X-Plane 10 displays 3-D Data about airflow (velocity and acceleration) and forces acting on the airplane in real-time. To bridge this to the classroom, students conduct a wind tunnel experiment to measure lift and drag of a wing profile, and the flow of air at different angles of attack. Afterwards, pairs of 2 students receive a 15 min flight lesson: take-off, level flight, slow flight with full flaps, acceleration back to cruise speed and an approach for a landing back on the airfield. One student acts as pilot flying; the other observes the patterns of airflow during maneuvers. They are expected to describe and explain their observation in a written lab report and link it to theory and the results of the wind tunnel experiment.

\section{Objectives and Assessment}

By qualitative analysis of their texts, interviews and partaking observation of students' interaction and discussion, we try to assess whether the flight model data can be interpreted correctly by students, and whether this learning arrangement can be used in a classroom setting. Special interest is in the discussion of students' concepts and mental models of the physics of flight before and after conducting the experiment. 


\author{
CERN's Media Lab Games in Upper Secondary Physics Education: \\ Overcoming Baek's Barrier to uptake Computer Games in Classrooms \\ Oliver Keller ${ }^{1}$, André Bresges ${ }^{2}$ and Nadine Westkamp ${ }^{2}$ \\ ${ }^{1}$ CERN Media Lab, Switzerland, ${ }^{2}$ University of Cologne, Germany
}

\title{
Introduction and Theory
}

Games are a mean for animals and humans likewise to acquire information about a complex environment and the physical, social or economical rules that dominates that environment. Generally speaking, the more an organism needs to learn in order to survive, the more it needs to play (Ackerman 2011). Authors like Marvin Prensky argue that digital game-based learning is a valuable mean to motivate today's students to learn; and that the exploitation of this mean is necessary to adapt to the changing nature and culture of today's learners (Prensky 2005). Empirical results show positive effects for the treatment of patients with chronic diseases (Lieberman 2001), and there is an abundance of examples for teaching computational science to already computer-affine target groups. Learning Games for the public sector receive mixed critique and are compared to "chocolate-covered broccoli" (e.g. Galarneau 2005) for hiding the bitterness of practicing math and science skills under a thin layer of sweetening graphics. For the public education sector in Korea, Baek (2008) has identified six reasons that inhibit the uptake of Computer Games in classrooms:

1. Inflexibility of the curriculum,

2. Negative effects of gaming,

3. Student's lack of readiness,

4. Lack of supporting materials,

5. Fixed class schedules and

6. Limited budgets.

On the other hand, Klopfer et al (2009) of MIT argue that games can engage players in learning that is specifically applicable to schooling, and there are means by which teachers can leverage the learning in such games without disrupting the worlds of either play or school.

\section{Concept and Implementation}

We try to develop a generic process to overcome Baek's 6 obstacles. Starting point are Games and Visualizations developed at CERN Media Lab to present particles, accelerators and radioactivity in an intuitive way repurposing advanced detector technologies developed at CERN. The game idea is supported by additional material and standard lab experiments, e.g. determination of the electron charge to mass coefficient e/m. Implementation into the curriculum takes place in an upper secondary physics class, and is documented by action research methodology, interviews and parttaking observations.

\section{Objectives and Assessment}

We try to answer the following questions:

1. What properties should typically be visualized and what quests can be developed when a scientific discovery gets transformed into a game based learning environment?

2. What game rules can be applied to game based learning scenarios for physics education?

3. How could a game based learning scenario be implemented in schools without being disruptive to standard classroom procedures?

4. How can gaming be part of student's assessment? 


\author{
Gaming, Visualization and Sharing Data \\ Initialize Learning in Educational Activities at CERN \\ Konrad Jende, Rolf Landua \\ CERN, Geneva, Switzerland
}

\title{
Introduction and Theory
}

The European Organization for Nuclear Research (CERN) realizes collaboration in particle physics and related subjects globally and peacefully under the premise of publishing all results [1]. Reviewing the diverse and widespread educational activities at CERN, three recurring concepts can be identified, which have the potential to initialize learning with modern media: Gaming [2], visualization [3] and sharing data [4]. Permanent and travelling exhibitions, visit tours on the CERN site, high school teacher programmes and websites emerge as mediators of such concepts.

\section{Concept and Implementation}

'Virtual Atom Smasher' [5] is an interactive educational online game. The game well reflects all of the above mentioned concepts. This is also valid for an interactive gaming simulation of particle collisions as exhibition content [6], which makes use of real experimental data from CERN's Large Hadron Collider. It was developed by CERN Media Lab [7], which also contributes to the production of high quality 3D models and renders for interactive applications and animations. Scientific visualization of CERN's experimental data is also being used in educational settings, such as, International Masterclasses [8] and shared with everyone through the Open Data portal [9].

\section{Objectives and Assessment}

Introducing the general public to the subject matter and methodology of particle physics and its associated disciplines, inspiring the young to take up science careers, supporting teachers and initiating the learning are the objectives of the concepts. They are assessed individually by surveys and feedback.

\section{References:}

1. CERN Council (2010): CONVENTION FOR THE ESTABLISHMENT OF A EUROPEAN ORGANIZATION FOR NUCLEAR RESEARCH. http://council.web.cern.ch/council/en/governance/Convention.html (Retrieved: 20 May 2015)

2. Gee, J.P. (2003): What Video Games Have to Teach Us About Learning and Literacy. In: ACM Computers in Entertainment 1 (1), 20-24.

3. Rapp, D. (2007): MENTAL MODELS: THEORETICAL ISSUES FOR VISUALIZATIONS IN SCIENCE EDUCATION. In: Visualization in Science Education, edited by Gilbert, J.K. Dordrecht: Springer.

4. Smith, T. (2014): On the road to Open Science. http://home.web.cern.ch/cern-people/opinion/2014/11/ road-open-science (Retrieved: 20 May 2015)

5. Homepage of Virtual Atom Smasher: http://test4theory.cern.ch (Retrieved: 16 May 2015)

6. Homepage of Interactive LHC Tunnel: http://medialab.web.cern.ch/content/interactive-lhc-tunnel (Retrieved: 20 May 2015)

7. Homepage of CERN Media Lab: http://medialab.web.cern.ch (Retrieved: 20 May 2015)

8. Bilow, U. \& Kobel, M. (2014): International Masterclasses: bringing LHC data to school children. In: EPJ Web of Conferences 71, 00017 (2014)

9. Homepage of CERN Open Data portal: http://opendata.cern.ch (Retrieved: 24 May 2015) 


\section{Game-Based Learning for Supporting Self-Confidence and Motivation of Female Physics Students \\ Bernhard Ertl \\ Donau-Universität Krems, Austria}

\section{Introduction and Theory}

Studies have shown that female students show less active classroom participation in STEM (sciences, technology, engineering, and mathematics) and particularly in physics courses (e.g. Jurik, Gröschner \& Seidel 2013). They also report a lower level of self-confidence - a phenomenon that can have negative impact on the development of interests as well as for career choices (e.g. Jurik et al. 2013). Stereotypes, particularly those of parents and teachers, can have a big impact on pupils' dispositions, especially stereotypes regarding females in the sciences as well as the respective attribution patterns (Martignon 2010). Furthermore, studies have shown that also parental support and dispositions influence the development of self-confidence of female students. This may be one of the reasons, why the share and the motivation of many female students in physics remained constantly low over the years although there were efforts like "Girls days" or "Girls do Science".

\section{Concept and Implementation}

This presentation deals with the issue of game based learning to support motivation and self-confidence of female students in lower secondary physics classes. It will discuss how game-based learning approaches can support girls' development of competencies as well as how they can counteract the development of stereotypes by enhancing appropriate selfevaluations. It will further present approaches and implementations of game based learning didactics in physics and bring examples from entertainment apps as well as evidence from the project Mit-Mut. Mit-Mut is an Austrian project originally dedicated to support girls' entrepreneurial skills in the ICT sector but also involves minigames about physics issues.

\section{Objectives and Assessment}

The contribution will discuss the evaluation of competencies acquired during game based learning. Evaluating game based learning activities has to find a balance between being able to grade students but without bringing the context of assessment to the game. Latter would shift the focus of the learners working with the game from performing a joyful activity to working with just another method of physics instruction. This kind of shift would have negative impacts to the students' motivation and mastery willingness. The contribution will conclude with the aspects self-evaluation during game based learning that could enhance students' self-confidence.

\section{References}

Jurik, V., Gröschner, A., \& Seidel, T. (2013). How student characteristics affect girls' and boys' verbal engagement in physics instruction. Learning and Instruction, 23(1), 33-42. doi: 10.1016/j.learninstruc.2012.09.002

Martignon, L. (2010). Mädchen und Mathematik. In M. Matzner \& I. Wyrobnik (Eds.), Handbuch MädchenPädagogik (pp. 220-232). Weinheim Beltz. 


\author{
Exploring Physics with Video Games \\ Aaron Titus ${ }^{1}$ and Francisco Esquembre ${ }^{2}$ \\ ${ }^{1}$ High Point University, USA, ${ }^{2}$ Universidad de Murcia, Spain
}

\begin{abstract}
Exploring Physics with Video Games combines classic arcade games, new app-based games, and physics instruction in one self-contained ePub readable (and playable!) on a computer, tablet, or smartphone. The book is for people of all ages who are interested in video games and even more interested in the physics behind them - the physics that makes objects move, bounce, shoot, explode, collide, and jump. Classic videos games like Asteroids, Space Invaders, Lunar Lander, Frogger, and Missile Command were all created with basic physics. Many recent games like Angry Birds, Flappy Birds, and Cut the Rope are based on a robust physics engine that performs calculations in the game. In some parts of the games, the physics is realistic. In other parts, the physics is 'fake'; that is, it follows laws of physics from a fictional universe or employs fictional technology in the game's world that is not apparent to the user.
\end{abstract}

This cutting-edge book uses games to teach physics and uses physics to teach games, making learning physics and gaming more fun than usual. As the first eBook to combine games and physics, we are exploring a new way to deliver fun, educational eBooks.

In this talk, we introduce the project and discuss the technical features and pedagogical approach that made the ePub book possible.

Each chapter of the book contains one popular game, followed by physics concepts, example calculations, and other versions of the game. After each game, we explore in greater depth the physics used in it. Frequently, popular video games use fake (i.e. unrealistic) physics to make the game easier or more fun to play. In these cases, we will follow up with a similar game based on correct physics. As a result, the reader will see how employing correct physics can dramatically affect gameplay. For most games, we use physics to write a program that plays the game automatically, thus achieving greater scores than are humanly possible.

All of the games and images were created with Easy JavaScript Simulations (EjsS, www. um.es/fem/EjsWiki), a free, open-source application and library created by Francisco Esquembre for writing and deploying simulations with mathematical modeling. EjsS programs run in a browser and in an app on computers, tablets, and smartphones. Tutorials and a library of EjsS simulations are available on ComPadre (www.compadre.org/osp).

High school and college teachers in physics, math, or computer science may want to weave our ePub, EjsS, and/or the book Physics for Video Games, created by Aaron Titus, into their instruction and class activities, including student projects, homework, and in-class exercises. We can envision teachers assigning chapters from our book and following up with questions, exercises, challenges, and exam problems. If our book is used with instruction in programming, then students should create their own games. A useful challenge is to think about how to automate gameplay to achieve the highest possible scores. 
PARALLEL SESSION MM:

\title{
MULTIMEDIA IN PHYSICS TEACHING AND LEARNING
}

\author{
Promoting Multimedia in Physics Teaching \\ Through the Flipped Classroom in Pre-Service Education \\ Vera Montalbano \\ University of Siena, Italy
}

\author{
Interactive CDF Diagrams in Introductory Physics Course \\ Farook Al-Shamali \\ Athabasca University, Canada
}

Liquid Physics:

The Wonderful World (Sometimes Turbulent) of Water's Films, Bubbles and Marbles Assunta Bonanno, Giacomo Bozzo and Peppino Sapia University of Calabria, Italy

Learning Mediated by Educational Hypermedia on the Phases of the Moon Adriano Luiz Fagundes ${ }^{1}$, Tatiana da Silva ${ }^{1}$ and Marta Feijó Barroso ${ }^{2}$ ${ }^{1}$ Federal University of Santa Catarina, Brazil, ${ }^{2}$ Federal University of Rio de Janeiro, Brazil

The Effect of Interactive Multimedia on Students' Achievement and Retention in NCE II Physics Students at FCT College of Education Zuba, Abuja Ibrahim Iliya Bisalla and Isiaku Haruna FCT College of Education, Zuba. Abuja. Nigeria

Non-Users, Lurkers, and Posters in the Online AP Teacher Community: Comparing Characteristics Determining Online Engagement Christian Fischer ${ }^{1}$, Kim Frumin ${ }^{2}$, Chris Dede ${ }^{2}$, Barry Fishman ${ }^{1}$, Arthur Eisenkraft ${ }^{3}$, Yueming Jia ${ }^{4}$, Janna Fuccillo Kook ${ }^{4}$, Abigail Jurist Levy ${ }^{4}$, Frances Lawrenz ${ }^{5}$ and Ayana McCoy ${ }^{3}$

${ }^{1}$ University of Michigan, USA, ${ }^{2}$ Harvard University, USA,

${ }^{3}$ University of Massachusetts at Boston, USA, ${ }^{4}$ Education Development Center, Inc., USA, ${ }^{5}$ University of Minnesota, USA 


\author{
Promoting Multimedia in Physics Teaching \\ Through the Flipped Classroom in Pre-Service Education \\ Vera Montalbano \\ University of Siena, Italy
}

\title{
Introduction and Theory
}

The increasing role of technology in contemporary society, as well as the rapid advancement of technology types and uses, requires major changes to methods of teaching. The use of a flipped classroom approach in a pre-service course can promote active learning in young teachers, enhance the critical thinking and obtain the maximum use of student-faculty time together (Keengwe 2014). This constructivist approach to teaching is an effective means of student-centered collaboration. The benefits are many, such as to maximize the learners' capacity to engage in small group discussion, project based learning, or problem solving tasks (Larcara 2014). Moreover, the face-to-face classroom time can be used for peer collaboration, inquiry, and project-based learning (Dickenson 2014).

\section{Concept and Implementation}

The flipped classroom was introduced in a course of Physics Lab Didactics in order to improve the number of proposed MM and the quality of their use in the teaching and learning process in laboratory. The course promoted active learning in all activities through the direct experience of young teachers. Furthermore, the flipped classroom approach was presented and discussed with the aim of clarifying the teaching process and in-training teachers were invited to explore how to implement this methodology in a class after their experience. Four different open source or free software were proposed (Audacity, Algodoo, GeoGebra and Tracker) with some examples of their use in physics teaching. The discussion on their implementation as tools in physics laboratory was postponed to the last lesson of the course.

\section{Objectives and Assessment}

The scope of the flipped classroom was achieved in advance because teachers started to discuss about specific uses of software during the time spent in laboratory. Their approach was very active and some application proposed by teacher is reported in detail. This pilot study shows a great potentiality of flipped classroom in pre-service education and it indicates that the use of MM in laboratory learning process can be improved following this approach.

\section{References}

Dickenson, P. (2014). Flipping the Classroom in a Teacher Education Course. Promoting Active Learning through the Flipped Classroom Model, 145-162.

Keengwe, J. (Ed.). (2014). Promoting Active Learning Through the Flipped Classroom Model. IGI Global. Larcara, M. (2014). Benefits of the Flipped Classroom Model. Promoting Active Learning through the Flipped Classroom Model, 132-144. 
Parallel Session MM

Room C (D Z007)

Contribution $4 \mathrm{C} 2$

Thu, 10 Sep, 14:20-14:35

\title{
Interactive CDF Diagrams in Introductory Physics Course \\ Farook Al-Shamali \\ Athabasca University, Canada
}

\begin{abstract}
Introduction and Theory
Teaching with multimedia is an emerging area in educational research driven by the accelerating advancement in communication technologies and by the explosion of online educational resources. Consequently, face-to-face (F2F) and distance education (DE) are evolving from their traditional practices and the gap between them is gradually closing. Learning physics has always been a challenge everywhere. An alarming gap between instructional goals and actual student learning in introductory physics courses was realized few decades ago, and incited pertinent research activity in physics education (McDermott, 1990). Such issue is of special interest in the DE model, which strives to position itself side by side with the F2F system. There is mounting evidence in the literature that online environments can be as effective as convectional classrooms, provided that a high quality online instruction is ensured (Tallent-Runnels, et al. 2006).
\end{abstract}

\section{Concept and Implementation}

The textbook is probably the most important educational resource in a typical physics course, but it is normally written with the conventional classroom in mind. In DE, the Study Guide becomes an essential component, complementing the textbook and providing detailed solutions to questions related to the subject matter.

PHYS 204 at Athabasca University is a calculus-based introductory physics course delivered online. Recently, the course went into a major revision that involves the production of a new Study Guide written in html with mathjax scripting of LaTeX code. The most important feature, however, is the interactive diagrams that replaced the traditional static figures and drawings. For this purpose, the "Animate" and "Manipulate" capabilities of the Mathematica software were used to produce CDF (Computable Document Format) files that can be embedded in the respective html pages. Students install the freely-available Wolfram CDF Player which provides a browser add-on to access the dynamic figures.

\section{Objectives and Assessment}

The concepts of kinematics and dynamics are not always intuitive. Visualizing the interaction between position, velocity and acceleration, especially in 2 and 3 dimensions, poses a challenge to many young learners. Students will appreciate the interactivity and observation of real time behaviors of projectiles, circular motions, collisions, etc. Some of the more involved diagrams act as virtual lab experiments due to the complexity of Mathematica coding and the ability to simulate real-life situations. The inclusion of interactive diagrams should take the Study Guide to the next level and give a richer meaning to online learning. Effectiveness of the new Study Guide will be assessed by analyzing students' satisfaction surveys and achievement results. 


\author{
Liquid Physics: \\ The Wonderful World (Sometimes Turbulent) of Water's Films, Bubbles and Marbles \\ Assunta Bonanno, Giacomo Bozzo and Peppino Sapia \\ University of Calabria, Italy
}

\title{
Introduction and Theory
}

The physics of fluids constitutes a very important topic whose treatment at level of secondary school and introductory university courses is usually restricted to the phenomenology of continuous laminar flows and to the main laws governing them. These arguments are certainly very important both on a conceptual perspective and in view of technological applications, since many systems of practical relevance involve the continuous flow of water-like fluids. Well-known examples are from engineering (e.g., transport of liquids in pipelines) and medicine (blood flowing in vessels) to cite some. However, there are many significant contexts in which drop-like and/or film-like forms of the liquids are involved. These include both relevant technological applications (for example inkjet printers and biotechnological and microfluidics devices) and issues of a wider interest such as those related to the problems of environmental pollution. Moreover, even in the case of continuous flows, there are situations far away from the laminar flow regime that have great relevance, both practically and conceptually: this is the case of turbulent motions.

\section{Concept and Implementation}

Previous considerations suggest the usefulness of a broader didactical perspective on the physics of fluids, that aims to introducing (at least qualitatively) new concepts, eliciting the interest of students for phenomena that, though less known, are extremely important from a technological point of view and in an interdisciplinary perspective. In this context, we have developed an interactive multimedia tutorial on the dynamical behavior of drop-like and film-like water aggregates, allowing students to get in touch with some peculiar water-based systems, either well known - as water films - or more exotic like "water marbles" (also known as "water beads"). Moreover, the tutorial features new original interactive visualizations of some turbulence phenomena, easily reproducible in a teaching lab.

\section{Objectives and Assessment}

The extensive use of advanced (though within the reach of a good teaching laboratory) visualization techniques (high-speed video shooting and UV-induced fluorescence contrast imaging) allowed us to implement a multimedia tool which presents both an illustrative character (aimed to capture the attention of learners) and more deep features of quantitative analysis of dynamic phenomena. Moreover, it gives students and teachers methodological and practical hints to independently conduct and analyze their own teaching experiments on proposed systems.

A first efficacy assessment for the didactical tool has been carried out within the context of some university guidance activities to students of high school's last year, implemented by the Department of Biology, Ecology and Earth Sciences of the University of Calabria. 
Parallel Session MM

Room C (D Z007)

Contribution 4C4

Thu, 10 Sep, 15:00-15:15

\author{
Learning Mediated by Educational Hypermedia on the Phases of the Moon \\ Adriano Luiz Fagundes ${ }^{1}$, Tatiana da Silva ${ }^{1}$ and Marta Feijó Barroso ${ }^{2}$ \\ ${ }^{1}$ Federal University of Santa Catarina, Brazil, ${ }^{2}$ Federal University of Rio de Janeiro, Brazil
}

\begin{abstract}
Introduction and Theory
Phases of the Moon is a digital educational material designed adopting the theory of cognitive load (CLT), reflections about the role of visualization in science education and the concept of digital learning object. This theoretical framework allows the assumption that both the characteristics of the visual resources as well as individual differences can influence computermediated learning.
\end{abstract}

\title{
Concept and Implementation
}

Hypermedia as a whole constitutes a learning object divided in smaller ones: "The Moon", "The Phases", "Synchronous Rotation" and "The Hemispheres". Each one of them was designed with multimedia resources in accordance with the instructional principles of CLT to provide visual support and aid learning. The first one discusses features of the Moon, a subject considered to be simple and familiar to most individuals. The second addresses lunar phases, a phenomenon that requires the understanding of the relative motion of Sun, Earth and Moon, the perception of how the Moon is illuminated by the Sun and how this enlightening is perceived on Earth to be fully understood, creating a great demand of intrinsic cognitive load and with a higher degree of reasoning complexity. The hypermedia provides some idealizations that provides some ease to the comprehension on the subject. The third learning object discusses the synchronous rotation of the Moon, a phenomenon that requires visualization and understanding of simultaneous rotational and translational motion. A model decoupling the two components can provide reduction on the complexity.

\section{Objectives and Assessment}

The hypermedia was assessed as a product, by science peers, with use of an online questionnaire, and it was considered of good educational quality.

It was also assessed by users, college students, by using it in an introductory undergraduate physics discipline of an STEM course; 77 students participated in the assessment process. Each learning object was evaluated separately. It is understood that one of the best ways to analyze whether individuals internalized the representations and models presented is by asking them to draw schemes or diagrams. Questionnaires and tests were used during a two month period.

The learning outcomes of the object "The Moon" assessed the knowledge of the Moon as a natural satellite of the Earth without proper light; a learning gain of $8 \%$ was obtained, confirming the hypothesis that it is already known by majority of students. The second learning object "The Phases" was assessed by asking students to represent a schematic diagram with lunar phase's sequences and to answer about the possibility of observing the Sun and the Moon according to the time of day, with an average percent gain of $40 \%$, providing a strong evidence on the instructional capacity of the interactive animation. The assessment on students' comprehension about the synchronous rotation of the Moon requested the explanation and the representation of motion dynamics, with an average gain of $50 \%$. A qualitative analysis allowed the identification of the effectiveness of the material. It is inferred that the characteristics of the hypermedia may have contributed effectively to the results, in accordance with previous results that points to the effectiveness of science learning materials supported by computational resources using interactive resources, an spatial organization of information and use of visual aspects as guides to the elaboration of materials. 


\section{The Effect of Interactive Multimedia on Students' Achievement and Retention in NCE II Physics Students at FCT College of Education Zuba, Abuja \\ Ibrahim Iliya Bisalla and Isiaku Haruna \\ FCT College of Education, Zuba. Abuja. Nigeria}

\section{Introduction and Theory}

Previous researches have shown that there is shrinking school programs in Physics in many nations, falling numbers of Physics graduates, a lowering of overall annual science budgets and a slowing in the rate of revolutionary inventions create the demand for new cheap, fast and effective teaching methods. Multimedia offers the potential to augment learning with a vibrancy that the old computer-based training couldn't easily achieve. Graphics illustrate each term, audio demonstrates the correct pronunciation, animations allow visualization of different parts of each whole and video shows everyday use.

\section{Concept and Implementation}

A total of 140 students were used for the study. Quasi-experimental design was used to investigate post-test and re-test of non equivalent groups i.e. experimental group was taught using interactive multimedia and control group was taught the same topic using the old computerbased method of teaching. The instrument used for data collection was physics Students" achievement test (PSAT). the data collected was analyzed by means of basic descriptive statistics such as mean, standard deviation (S.D) was placed in tables as appropriate. Then the research questions were answered and the research hypotheses were tested using the t-test statistics.

\section{Objectives and Assessment}

The purpose of this study was to investigate the effect of interactive multimedia on students' achievement and retention in NCE II physics students at Federal Capital Territory FCT Abuja. Three(3) research questions and three hypotheses were formulated. The findings revealed that there was significant difference in the achievement and retention of students exposed to interactive multimedia method of instruction. It was recommended that Governments should urgently pursue the implementation of interactive multimedia mode of instruction in all colleges of Education, and provide all the necessary facilities, human and materials in order to achieve the best learning outcome in the world of computer Technology or revolution. 
Parallel Session MM

Room C (D Z007)

Contribution 4C6

Thu, 10 Sep, 15:40-15:55

\author{
Non-Users, Lurkers, and Posters in the Online AP Teacher Community: \\ Comparing Characteristics Determining Online Engagement \\ Christian Fischer ${ }^{1}$, Kim Frumin ${ }^{2}$, Chris Dede ${ }^{2}$, Barry Fishman ${ }^{1}$, Arthur Eisenkraft ${ }^{3}$, Yueming Jia ${ }^{4}$, \\ Janna Fuccillo Kook ${ }^{4}$, Abigail Jurist Levy ${ }^{4}$, Frances Lawrenz ${ }^{5}$ and Ayana McCoy ${ }^{3}$ \\ ${ }^{1}$ University of Michigan, USA, ${ }^{2}$ Harvard University, USA, \\ ${ }^{3}$ University of Massachusetts at Boston, USA, ${ }^{4}$ Education Development Center, Inc., USA, \\ ${ }^{5}$ University of Minnesota, USA
}

\title{
Abstract
}

This paper describes initial findings from a longitudinal NSF-funded study on teacher learning connected to the redesigned Advanced Placement (AP) science examinations in the United States. In response to a National Research Council Report (NRC, 2002), the redesigned AP courses and examinations emphasize scientific inquiry, reasoning, and depth of understanding. To support teachers, College Board (the AP provider) offers a variety of professional development (PD) options including the online AP Teacher Community (APTC), which provides a platform for discussing teaching strategies and sharing resources. Initial research indicates that, among the PD options studied, APTC participation has the largest, direct, and positive effect on both teaching practices and students' AP scores (Fishman et al., 2014). In this paper, we deepen our exploration of teacher participation in the APTC. For instance, characterizing lurkers as valid and active participants (e.g., Edelmann, 2013) engaging in legitimate peripheral participation (Lave \& Wenger, 1991) lead to the hypothesis that lurkers and posters have similar characteristics. However, in order to generalize to the AP teacher population, exploration of characteristics that can predict APTC participation is needed.

Data for this quasi-experimental study is collected through a web-based survey sent to every AP science teacher (Physics, Chemistry, Biology) asking about their interactions with the APTC and other PD options, teaching background, AP science instruction, school context, and concerns about the AP redesign. This proposal uses data from Biology and Chemistry teachers $(\mathrm{N}=4,899)$ collected in 2014 . The 2015 data from AP Physics teachers $(6,012$ surveys) is being collected in June 2015. The presentation and paper submission will add and compare the 2015 AP Physics data to the 2014 AP data.

Analytical methods include ANOVA and Kruskal-Wallis $\mathrm{H}$ tests for detecting differences between characteristics of lurkers (posting less than $5 \%$ of total time), posters (posting at least $5 \%$ of total time), and non-users (no APTC participation), as well as multi-group comparisons using Tukey-Kramer and post-hoc Mann-Whitney tests. Using the same variables logistic regression and ordered logistic regression predict the odds of being a lurker or poster as well as a non-user compared to a lurker or poster.

Preliminary analysis suggests that most characteristics (e.g., teachers' knowledge and experience, AP workload, AP practices enactment) are significantly associated with changes in the odds of being posters or lurkers compared to non-users indicating that the APTC attracts a unique teacher population. However, most teacher, teaching, and school characteristics do not help us determine whether a teacher is a poster or lurker. Given the unique opportunity to examine online participation patterns, this study indicates that researchers investigating online teacher communities need to account for the specificity of its users. 



\title{
INVITED SYMPOSIUM QP:
}

\author{
MULTIMEDIA IN TEACHING AND LEARNING QUANTUM PHYSICS \\ Antje Kohnle (Chairperson), University of St. Andrews, UK \\ Rainer Müller (Discussant), Technische Universität Braunschweig, Germany
}

Quantum physics holds a fascination for many students, but its mathematical complexity, counterintuitive results and phenomena far-removed from everyday experience can present major barriers. Multimedia resources can help to overcome these issues through visualization, supporting model-building, focusing on fundamental ideas and making the invisible visible. This symposium will give an overview of different multimedia resources for the teaching and learning of quantum physics, including online curricular materials, visualizations, animations, interactive simulations and screen experiments. The materials are aimed at different target audiences ranging from the general public to school and university students.

\section{Multimedia Resources for Quantum Physics Instruction: Opportunities and Challenges Antje Kohnle ${ }^{1}$, Carl Humphreys ${ }^{1}$ and Mark Paetkau ${ }^{2}$ ${ }^{1}$ University of St Andrews, United Kingdom ${ }^{2}$ Thompson Rivers University, Canada}

\author{
Making Quantum Mechanics Visual and Interactive: \\ Open Source Physics Based Curricular Material \\ Wolfgang Christian \\ Davidson College, USA
}

New Ways to Reimagine Quantum Physics: From 3D Animations to Design and Applied Art Julien Bobroff ${ }^{1}$, Frédéric Bouquet ${ }^{1}$ and Camille Jutant ${ }^{2}$ ${ }^{1}$ Université Paris-Sud \& CNRS, UMR 8502, Orsay, France ${ }^{2}$ Université Lumière Lyon 2, France

Interactive Screen Experiments With Single Photons Patrick Bronner ${ }^{1}$, Andreas Strunz ${ }^{1}$, Christine Silberhorn ${ }^{2}$ and Jan-Peter Meyn ${ }^{1}$ ${ }^{1}$ University Erlangen-Nuremberg, Germany, ${ }^{2}$ University Paderborn, Germany

\author{
Learning Quantum Information With Online Resources \\ Torsten Franz \\ TU Braunschweig, Germany
}

\author{
Visualization of the Structure of the Periodic Table of Elements \\ Based on Group Theory \\ Stefan Heusler \\ University of Münster, Germany
}




\author{
Multimedia Resources for Quantum Physics Instruction: \\ Opportunities and Challenges \\ Antje Kohnle ${ }^{1}$, Carl Humphreys ${ }^{1}$ and Mark Paetkau ${ }^{2}$ \\ ${ }^{1}$ University of St Andrews, United Kingdom \\ ${ }^{2}$ Thompson Rivers University, Canada
}

\title{
Introduction and Theory
}

Multimedia resources for quantum mechanics can provide tailored visual representations to make the invisible visible and facilitate the development of productive mental models. They can leverage the use of analogy to help learners gain quantum mechanics intuition. They can give students experiences of carrying out virtual and simulated quantum-mechanical experiments that would be too expensive or complex to carry out in a real lab. They can allow students to compare and contrast the behaviour of classical particles and quantum objects under the same experimental conditions.

\section{Concept and Implementation}

This presentation will give an overview of the symposium contributions in terms of these opportunities and challenges. We will also describe recent work on the QuVis Quantum Mechanics Visualization project (www.st-andrews.ac.uk/physics/quvis). QuVis consists of research-based interactive simulations with accompanying activities for the learning and teaching of quantum mechanics. Over the past year, we have substantially extended the number of HTML5 simulations available which run on both tablet-based devices and desktop computers. We have also enhanced the QuVis website functionality in terms of search capabilities for simulations for particular topics and levels.

\section{Objectives and Assessment}

With the aim of enhancing student engagement with the simulations, we have included goaland-reward structures using multiple challenges aligned with the learning goals. We have investigated the effect of the revised simulation format on students' experience using surveys completed by students directly after working with a simulation and by comparing time-ontask for different formats. Factors identified that impact students' engagement with the simulations include level of interactivity, clearly defined goals, rewarding progress, real-world applications and on-demand explanations. 


\section{Making Quantum Mechanics Visual and Interactive: Open Source Physics Based Curricular Material Wolfgang Christian \\ Davidson College, USA}

\section{Introduction and Theory}

The teaching of quantum mechanics has remained relatively frozen in time since its inception, despite recent work assessing and improving the conceptual understanding of students and despite availability of computer simulations. Students, therefore, often see quantum mechanics in terms of misleading or incomplete visualizations, as one dimensional and time independent, and devoid of almost any connection with classical physics.

\section{Concept and Implementation}

To address this situation, we have produced and class-tested interactive Open Source Physics (OSP) based curricular material in support the teaching of quantum mechanics. These exercises address both quantitative and conceptual difficulties encountered by many students in such topics as wave function shape, momentum space, time evolution, and classical/quantum correlations.

\section{Objectives and Assessment}

Open Source Physics materials are adaptable and can be used at a variety of levels with a variety of pedagogies. For example, Gaungtian and Singh have used our QM Measurement simulation in a Quantum Interactive Learning Tutorial (QuILT) and have shown that students using this QuILT have an improved interpretation of the shapes of the eigenfunctions for different operators corresponding to different physical observables and can better apply the projection method to calculate the probabilities of measuring a physical observable. Examples of OSP Quantum Mechanics curricular materials and future directions of the OSP Project, include the distribution of material through the AAPT-ComPADRE website $<\underline{\mathrm{http}} / / /$ www.compadre.org/OSP/> and through ePubs on Apple iTunes and Google Play stores, will be presented. 


\author{
New Ways to Reimagine Quantum Physics: \\ From 3D Animations to Design and Applied Art \\ Julien Bobroff ${ }^{1}$, Frédéric Bouquet ${ }^{1}$ and Camille Jutant ${ }^{2}$ \\ ${ }^{1}$ Université Paris-Sud \& CNRS, UMR 8502, Orsay, France \\ 2Université Lumière Lyon 2, France
}

\title{
Introduction
}

Our group ${ }^{1}$ has developed various outreach and teaching tools about quantum physics involving new kinds of interdisciplinary collaborations between physicists, designers, multimedia and graphic artists. In this presentation, we will focus on two examples, one more pedagogical (« Quantum Made Simple») and one more on the outreach and art-science side («Quantum Design »).

\section{Concept and Implementation}

"Quantum Made simple"2 consists of a set of 13 online 3D pedagogical animations about quantum physics gathered in a website and displayed in youtube and various Wikipedia articles. These animations address both fundamental quantum properties (duality, quantization, spin, tunneling...) and more recent research topics (grapheme, decoherence...) or modern techniques (photoemission, pump-probe techniques, crystallography...). They were developed by a team composed of physics researchers in these fields and graphic, web and sound designers. They are now widely used in France both for outreach (conferences, exhibits, science fairs) and as introductory and visual aids for quantum physics teaching in high schools and universities.

"Quantum Design"3 is the result of collaboration between physicists and a design school, the ENSCI-Les Ateliers. The designers produced videos, tools, video games and other multimedia contents to engage the general public with quantum physics. These productions offer a more intuitive, aesthetic and tangible approach to the quantum world, which supplements the more pedagogical "Quantum Made Simple" animations.

\section{Analysis}

A sociological and semiological study ${ }^{4}$ of the collaboration with designers in the "Quantum Design" project revealed that such productions renew not only the content but also the format and process of outreach or teaching. We indeed found that such collaborations produce new types of multimedia and outreach tools, both by their format, by the way they represent physical properties, and by the way they are displayed in front of an audience ${ }^{4}$. This could help renew the tools used for teaching physics and the teaching process itself.

We are now developing similar case studies about our other productions, including "Quantum Made Simple" animations but also pop-up and foldings, comic strips, and animated gifs illustrating various aspects of quantum physics ${ }^{1}$.

\section{References}

1. www.PhysicsReimagined.com : all our contents are available for free download and reuse

2. www.QuantumMadeSimple.com

3. www.DesignQuantique.fr

4. C. Jutant, J. Bobroff, “Objets de médiation de la science et objets de design. Le cas du projet Design Quantique ", Communication \& Langages, p.9, Vol.183 (2015) 


\author{
Interactive Screen Experiments With Single Photons \\ Patrick Bronner ${ }^{1}$, Andreas Strunz ${ }^{1}$, Christine Silberhorn ${ }^{2}$ and Jan-Peter Meyn ${ }^{1}$ \\ ${ }^{1}$ University Erlangen-Nuremberg, Germany, \\ ${ }^{2}$ University Paderborn, Germany
}

\begin{abstract}
Introduction
Single photons are suitable to observe the fundamentals of quantum physics. Quantum optic experiments have been successfully used for undergraduate university courses. We develop interactive quantum optic screen experiments to make such experiments available to students who do not have access to a real laboratory. For the education experiments, the same methods and optical components are applied that are used in a modern scientific laboratory. The measuring data recorded are comparable to results from current scientific publications.
\end{abstract}

\title{
Concept and implementation
}

For the interactive screen experiments the experimental setup is photographed in different settings. The photos are linked in a program with real data from the experiment. The user can change the settings of the experiment and follow the measurement results. The quantum behaviour of single photons can be observed in two modes: single events and events per second. The following interactive experiments are available with explanations on our homepage www.quantumlab.de: proof of the existence of the photon, quantum-random-number generator, photon statistics, quantum-cryptography, single photon interference, quantum-eraser, Hong-Ou-Mandel dip, entanglement with violation of Bell inequalities.

\section{Objectives and Assessment}

Based on the interactive experiments we developed quantum mechanics curricular material which can be used at high-schools (see classroom video) or as introduction in quantum physics classes at university. As an addition to the interactive, two optical training laboratories were opened at Erlangen-Nuremberg University. In the first laboratory, experimental optical basics are taught which are needed in the second laboratory for the setup of experiments with single photons.

\section{Literature}

P. Bronner, A. Strunz, C. Silberhorn, and J. P. Meyn, "Interactive screen experiments with single photons," European Journal of Physics 30 (2), 345-353 (2009).

P. Bronner, A. Strunz, C. Silberhorn, and J. P. Meyn, "Demonstrating quantum random with single photons," European Journal of Physics 30 (5), 1189-1200 (2009).

classroom video: https://youtu.be/4zB7WNNzTCI 


\title{
Learning Quantum Information With Online Resources \\ Torsten Franz \\ TU Braunschweig, Germany
}

\begin{abstract}
Introduction and Theory
Quantum information is a current modern field of research, utilizing the properties of quantum systems to perform information tasks. It is a good candidate for an introductory topic on quantum physics, as it allows learning basic principles of quantum physics with concrete and motivating examples, such as quantum cryptography and quantum computing.

We are developing an online course system (www.quanth-physik.de) on the topic of quantum information. Our target audience is university students, both physics students and prospective teachers. We will report on the development and status of the project and on results from testing with students in different universities.
\end{abstract}

\section{Concept and Implementation}

Our course is designed to cover material suitable for a one-semester quantum physics course, covering the topics "Quantum uncertainty", "Bell inequalities", "Quantum cryptography" and "Quantum computing". The core elements that have been implemented so far are in the format of still texts and pictures, where we are currently adding the use of animations and interactive elements.

Students use the online material to learn individually, which is accompanied by one just-intime lecture per month. This means, that the students are required to post their questions prior to the lecture and only these questions will then be addressed.

The lecture has been given in this just-in-time format twice at the University of Braunschweig and parts have been used in a lecture on the same topic at the University of Hannover. All implementations have been evaluated.

\section{Objectives and Assessment}

The target of our investigation was to determine, to which extend students are able to work with the provided material and whether there are differences in motivation and learning outcomes. The learning outcomes were evaluated using a multiple-choice test and an analysis of video-interviews. The motivation was evaluated using a general motivation inventory, either as post-semester or as pre-post-intervention design.

In our talk, we will present the concept of our online course together with selected results from the evaluations. 


\title{
Visualization of the Structure of the Periodic Table of Elements Based on Group Theory \\ Stefan Heusler \\ University of Münster, Germany
}

\begin{abstract}
Introduction and Theory
Atomic models commonly used at high-school may be appropriate for teaching some basic physical properties of the hydrogen atom. Models for the structure of the complete periodic table based on group theory are know in the scientific literature $[1,2,3]$, however, such a group theoretical approach seems to be out of reach at high-school level due to the limited mathematical background.
\end{abstract}

\section{Concept and Implementation}

The key idea of our approach to quantum physics is the introduction of visualizations as a powerful representation of mathematical concepts. These visualizations may give access to subtle physical ideas such as quantum entanglement [4], or the measurement process for the simplest quantum state, the qubit $[5,6]$ even at high-school level, avoiding or at least complementing the abstract mathematical formalism. In this talk, we discuss our visualization of the group theoretical approach to the periodic table. One central idea is the link between the number of nodal lines of a (quantum) state, its symmetries, and highest-weight representations.

\section{Objectives and Assessment}

Based on the visualization scheme, we develop an E-Learning environment based on computer graphics [7] which can be used both at high-school and in introductionary quantum physics classes at university. We show that only a very limited extention of the commonly used models at high-school is sufficient to explain basic properties of the periodic table as e.g. the octed rule, or the chemical properties of lanthanoides. Further extensions of the developed visualization scheme leads to new models for selection rules, ortho-and para states, and other basic properties of quantum chemistry.

\section{References}

1. Scerri, E. The Periodic Table: Its Story and Its Significance. Oxford University Press, New York, NY, 2007.

2. Kibler, M.R. On the Use of the Group SO $(4,2)$ in Atomic and Molecular Physics. Mol. Phys., 102:1221-1230, 2004.

3. Kibler, M.R. A group-Theoretical Approach to the Periodic Table: Old and New Developments. In D.H. Rouvray and R.B. King, editors, The Mathematics of the Periodic Table. Nova Science, NY, pp. 237-263, 2006.

4. S. Heusler, Quantendimensionen, DVD-ROM, Klett-Verlag, Sciencemotion (2010)

5. W. Dür, S. Heusler, Visualizing the invisible: The qubit as a key to quantum physics, Phys. Teach. 52, 489 (2014).

6. W. Dür, S. Heusler, What we can learn about quantum physics from a single qubit, arXiv:1312.1463 [physics. ed-ph] - http://arxiv.org/abs/1312.1463

7. S. Heusler, Quantenspiegelungen, DVD-ROM, Klett-Verlag, Sciencemotion (publication date: 2016) 
PARALLEL SESSION ILA:

\title{
CONCEPTS TO INITIALIZE LEARNING ACTIVITIES WITH MODERN MEDIA
}

\author{
The Impact of ICT and Multimedia \\ Used to Flip the Classroom (Physics Lectures) via Smart Phones And Tablets \\ Beáta Jarosievitz \\ Dennis Gabor College, Hungary \\ Physics of Money Dynamics \\ ${ }^{1}$ Francesco Scerbo, ${ }^{1}$ Elena Scordo, ${ }^{1}$ Laura Vero and ${ }^{2}$ Salvatore Giampà \\ ${ }^{1}$ Liceo Scientifico "L.Siciliani" Catanzaro, Italy, ${ }^{2}$ Unical- University of Calabria, Italy
}

Home Made Spectrophotometer for a Laboratory Bridging Optics and Modern Physics Pasquale Onorato, Massimiliano Malgieri and Anna De Ambrosis University of Pavia, Italy

\begin{abstract}
Spectral Analysis of Organic Solar Cell Material Using Multimedia Devices for Physics Teaching

Zoltán Csernovszky ${ }^{1}$ and Ákos Horváth ${ }^{2}$

${ }^{1}$ Secondary School Kölcsey, Budapest, Hungary,

${ }^{2}$ University of Roland Eötvös ELTE, Budapest, Hungary
\end{abstract}

\begin{abstract}
Integrated Use of Scratch and EjsS for Primary Teachers' Preparation in the Perspective of Pedagogical Implications of Coding Skills

Peppino Sapia ${ }^{1}$, Giacomo Bozzo ${ }^{1}$ and Claudio De Luca ${ }^{2}$

${ }^{1}$ University of Calabria, Italy, ${ }^{2}$ University of Basilicata, Italy
\end{abstract}

Multimedia to Gain the Physics Description of Motion in the Early Years of High School

Stefano Vercellati and Marisa Michelini

University of Udine, Italy 


\section{The Impact of ICT and Multimedia \\ Used to Flip the Classroom (Physics Lectures) via Smart Phones And Tablets \\ Beáta Jarosievitz \\ Dennis Gabor College, Hungary}

\section{Introduction and Theory}

Based on the conclusions of my previous research activity, and based on different publications it has become clear that science lectures (especially Physics) should be made more colourful, attractive and interesting. Unfortunately students are not really involved in hands on experiments, and the background of the schools and university laboratories are very different. If we want to let our students leave universities, colleges or even secondary schools with an adequate knowledge and with applicable skills in Physics, we should take advantage of the ICT, multimedia and new devices and their applications (Jarosievitz, 2011, 2009). All these new possibilities can be used only if we have a well constructed hypothesis which is also researched with new educational methods.

\section{Concept and Implementation}

Before implementing the "peer instruction", an innovative teaching method developed by Prof. Eric Mazur (1997) I have formulated the following goals why I would like to use the new techniques and methods:

- to improve the memory of students (Inquiry-based learning),

- to improve their understanding (Nicol \& Boyle, 2003),

- to create interactive lectures (use of the multimedia tools and simulation), to encourage student's motivation,

- to make the lessons more enjoyable (students should be involved in real experiments),

- to improve student attendance,

- to improve concentration of the students,

- to evaluate students.

The hypothesis that the "peer instruction" method helps to reach these goals is also covered by previously published papers like: (e-Skills EU Newsbrief, March 2010)

Implementation steps:

- students read some papers, articles in advance of the lectures,

- they pay attention to the lectures,

- take part in the demonstration (experiment presented for class),

- use ICT and free programs (e.g: http://www.socrative.com/)

- students turn to their neighbor and discuss their topic

- run the simulation apps on their devices, make conclusions and answer to their questions.

During the implementation all activity is controlled by the lecturer and also the cooperation method is used.

\section{Objectives and Assessment}

As conclusion of the research I am confident, that the use of the ICT and Multimedia has a big impact in:

- modernize teaching,

- investigate students' motivation

- follow the mobile revolution,

- use mobile for good purposes,

- follow different high-tech developments.

"When looking at the current widespread diffusion and use of ICT in modern societies, especially by the young - the so-called digital generation - then it should be clear that ICT will affect the complete learning process today and in the future" (Punie, Zinnbauer and Cabrera, 2012) 


\author{
Physics of Money Dynamics \\ Francesco Scerbo ${ }^{1}$, Elena Scordo ${ }^{1}$, Laura Vero ${ }^{1}$ and Salvatore Giampà ${ }^{2}$ \\ ${ }^{1}$ Liceo Scientifico "L.Siciliani" Catanzaro, Italy, ${ }^{2}$ Unical- University of Calabria, Italy
}

\begin{abstract}
Introduction and Theory
Within the teaching of physics, and in particular within classical mechanics, both momentum and energy conservation laws are considered in curriculum, but too often their use is limited in resolving traditional physics problems and situations. In this work we want to demonstrate how kinetic energy conservation law in elastic collisions can be successfully applied in a different scenario to model effective money distribution among individuals (families) in a stable economy.
\end{abstract}

\title{
Concept and Implementation
}

The starting point of our approach is a straightforward parallelism between economic agents interaction and mechanical interaction in multi-particles physical systems: indeed due to the large number of individuals exchanging money, an economical stable ensemble of agents can be seen as a thermodynamic system. Each economical trade behaves like a physical elastic interaction between two particles. In a stable economic system total money amount is constant, this is equivalent to impose energy conservation in the physical system. We assume that agents exchange a fraction of their money by pairs and in each exchange total amount of money is conserved: in physical terms each interaction is considered elastic and kinetic energy is conserved. In our model the amount of money Dr exchanged in each trade depends on the two parameters, $y$ fraction of personal richness involved in the trade and e risk propension through the linear formula: $\mathrm{Dr}=(1-\mathrm{y})[\mathrm{ri}-(1-\mathrm{e}) \mathrm{rj}]$ with ri and rj initial money amount of the two interacting agents. In each iteration whole population is divided in pairs exchanging money. Just after a few iterations money distributions becomes highly skewed approaching a power law. In a refined version a quadratic model for Dr allowed to take into account the reduced risk propension for higher richness. A PC-based software has been developed allowing for interactive simulations.

\section{Objectives and Assessment}

This classroom project has been conducted during extra-hours with the aim to bring student to the understanding of a physical principle through its application to a non standard problem. Parallelism between particles interactions and money exchanges helped students in acquiring the concept of "physical modeling". Infact teacher initially started from the simplest possible system: only two agent, with same money amount, exchanging money. Students easyly realized that single agent changing money amount is fully compatible with conservation of total money amount. Furthermore teacher showed that increasing agents population always brings towards the "same", statistically speaking, money distribution.

Computer simulations proved to be very addictive and stimulating for students, who fully exploited software capabilities, and will be shown during the presentation.

G. Albi - Econofisica. Leggi Universali. Particelle ed agenti economici - Dip. di Matematica - Università di ferrara 06/03/2012 https://laboratoriopls.files.wordpress.com/2012/01/lab06_ariosto.pdf

M. Patriarca, A Chakraborti - Kinetic exchange models. From molecular physics to social science - Am. J. Phys. 81 (8), August 2013

A. Chatterjee, B.K. Chakrabarti, S.S. Menna - Pareto lae in kineti model of market with random saving propensity - Physica A 335 (2004) 155-163

A. Chakraborti, B.K. Chakrabarti - Statistical mechanics of money: how saving propensity affects its distribution - Eur. Phys. J.B. 17, 167-170 (2000)

G. Toscani - La curva della ricchezza - Dip. di Matematica , Università di Pavia - 12/06/2014 http://wwwdimat.unipv.it/vitali/Stage2014-rete/La curva della ricchezza.pdf 


\section{Home Made Spectrophotometer for a Laboratory Bridging Optics and Modern Physics Pasquale Onorato, Massimiliano Malgieri and Anna De Ambrosis University of Pavia, Italy}

\section{Introduction and Theory}

We present a simple methodology employing digital photography and image processing techniques that can be used, both in introductory physics laboratory and in high school, to obtain quantitative measurements of position and intensity in optic experiments by means of a CCD commercial camera or a cell-phone.

Our idea was to prepare low cost experiments, based on home made equipment, suitable to be used by groups of students with an inquiry approach.

\section{Concept and Implementation}

Aiming at bridging optics and modern physics, we designed low cost spectrometers, based on the use of either transmission or reflection diffraction gratings. This simple equipment was used to measure the wavelength of visible lines of Balmer series from the hydrogen atomic spectrum and estimate the value of Rydberg's constant with an error difference of few tenths percent. It was also employed to evaluate the Planck's constant by measuring the wavelength of the light emitted by diodes of various colors, and to study some peculiar aspects of photoluminescence.

\section{Objectives and Assessment}

The experiments have been tested with high school students and with student teachers in a postgraduate course for physics teacher education. We collected data on students' ideas from the worksheets completed during the experimental activities; discussions during and after the experiments; and the reports they prepared afterwards. Results will be discussed in detail at the conference. 


\author{
Spectral Analysis of Organic Solar Cell Material \\ Using Multimedia Devices for Physics Teaching \\ Zoltán Csernovszky ${ }^{1}$ and Ákos Horváth ${ }^{2}$ \\ ${ }^{1}$ Secondary School Kölcsey, Budapest, Hungary, \\ ${ }^{2}$ University of Roland Eötvös ELTE, Budapest, Hungary
}

\begin{abstract}
The lecture presents a simplified model of solar cells and photosynthesis relevant from an energetic and biological perspective. With the activities for interactive whiteboard and other mobile medias, we present the Hungarian Educational Platform "RevoEd". This application helps to illustrate and familiarise quantified energy levels and semiconductor bands for Secondary School Physics courses.

For these reasons, the comparative spectral analysis of the absorption spectra of the solutions containing leaf pigments and materials of organic solar cells will be based on the results of a homemade spectroscope. We analyse the spectra of different lamps and their absorption. With all spectral analysis we contribute to an open source web database, "Spectral Workbench".

We compare the impact of the photoeffect taking place within the solar cell, i.e. the energy exchange of the photons with the processes taking place inside a leaf during photosynthesis. In the examination of the solar cell we determine the cell's voltage-current characteristics and the maximum value of efficiency in terms of incident light intensity to compare the efficiencies of the photosynthesis of different pigments.

We underline that during photosynthesis, the change in the electrostatic energy of the molecules does not result in an electric current. However, the idea of using photosynthesis to convert sunlight into electrical power appeared in the 1970s.

As an interdisciplinary application, we expose a dye-sensitized solar cell, which use raspberry juice as natural dyes as light-harvesting pigments. In this type of low-cost and homemade solar cell, the excited electrons are transferred to the conduction band of a wide bandgap semiconductor. We suggest some possible exploitations of the project activities with different multimedia devices.

To show the interaction of theory and experience the educational project activities based on homemade tools (spectroscope and dye-sensitized solar cell) and their analysis with multimedia devices can open an exciting way to the educational development. With its prepared and various activities, the Platform "RevoEd" offer a creative and ideal working place for the teachers and for all types of classes.
\end{abstract}




\author{
Integrated Use of Scratch and EjsS for Primary Teachers' Preparation \\ in the Perspective of Pedagogical Implications of Coding Skills \\ Peppino Sapia ${ }^{1}$, Giacomo Bozzo ${ }^{1}$ and Claudio De Luca ${ }^{2}$ \\ ${ }^{1}$ University of Calabria, Italy, ${ }^{2}$ University of Basilicata, Italy
}

\title{
Introduction and Theory
}

The usefulness of multimedia tools for the teaching/learning processes of scientific disciplines is well established at all grade levels. This is particularly true in those contexts where learners can take great advantage from the interactive visualization of the relations among the significant variables of a physical system. An important example is represented by the study of the motion of bodies in the primary school. In fact, the space-vs-time and velocity-vstime representations constitute a topic widely recognized as crucial by educational research. On the other side, the "Computational thinking" is increasingly recognized at primary level as an ability helping children to be articulate and think logically. It is a skill children must be taught if they are to be ready for the workplace and able to participate effectively in the digital world. In this context, "Coding" is becoming increasingly a key competence, which will have to be acquired by all young students. It is part of logical reasoning and represents one of those key skills that are now called "21st Century skills".

\section{Concept and Implementation}

The described conceptual framework strongly requires that the primary school teachers' preparation also aim to the development of skills concerning both the critical use of multimedia tools and the practice of Coding. Prospective primary teachers are usually lacking of a formal instruction as regards programming languages (at least in Italy). Therefore, they can take advantage from the employ of authoring tools allowing them to build up their own interactive simulation with very little prior knowledge of programming. The software "Easy Java/Javascript Simulations" (EJsS) is certainly one of the most suitable in helping non-programmers create interactive simulations for teaching purposes. Nonetheless, some familiarity with the basic concepts of the logic of programming is still necessary. A teaching tool very useful for this purpose consists of the "Scratch" software, a visual tool designed to enable computing novices without prior programming knowledge, to acquire the basic coding-related knowledge and skills.

\section{Objectives and Assessment}

In this work, we describe the experience of introducing the synergic employ of "EJs]" and "Scratch" in a fourth-year course of the degree program for Primary Teachers Preparation at the University of Basilicata (Italy). Students taking the "Physics Education and History of Physics" course where challenged to create simple kinematics simulations using Scratch, and then to implement the corresponding interactive graphical representations $s(t)$ and $v(t)$ by using EjsS. The direct observation of students' activity and the analysis of a questionnaire given to them suggest that the joined employ of the two mentioned software tools can be effective in allowing computing-novices prospective teachers to quickly develop the basic knowledge and skills needed to make a critical and autonomous use of multimedia tools in their future teaching activities. 


\author{
Multimedia to Gain the Physics Description of Motion \\ in the Early Years of High School \\ Stefano Vercellati and Marisa Michelini \\ University of Udine, Italy
}

\begin{abstract}
Introduction and Theory
Research in physics education has extensively addressed the main learning knots in kinematics and, in particular the persistent common ideas on motion description with the lack of formalization process in a coherent physics framework.

The creation of multimedia tools and instruments that are even more intuitive and close to the final user needs provided an increasing diffusion of multimedia tools in schools but, to be effective, the use of these instruments had to inserted in educational environment able to provide an effective use of them for the learning process in a physics framework.
\end{abstract}

\title{
Concept and Implementation
}

The educational activities described took place during the first year of a technical secondary school. To introduce students to kinematics, a non-reductionist approach based on the representation of displacement vectors at constant intervals of time was adopted [Karplus, 1977]. The description of motions was proposed directly in two dimension avoiding starting the description of the motions with the discussion of the one-dimensional case where displacements, velocities and accelerations are treated as scalars instead of vectors.

The proposed learning path was designed using an Inquiry Based approach where students are active in the process of construction of their knowledge and in which an intensive use of Real Time Laboratory activities are integrated with video analysis software (Tracker) to promote the bridging construction of the formal thinking. Discussions on interactive white board produce a cooperative comparison of ideas and interpretative models. The whole length of the module of formative intervention proposed is of 10 hours.

\section{Objectives and Assessment}

Data were collected from student worksheets, recording of the laboratory activity and preliminary and posttest proposed at the beginning and at the end of the learning path.

Preliminary test was constituted by open questions used to have a picture of the entry level of the class. Posttest was instead constructed as a multiple-choice test where the questions are similar to the ones proposed in the pretest and the wrong options for each question are constructed starting from the more common ideas highlighted in the students' answers to the pretest. In this way, worksheets allow to follow the development of the evolution of students' model in the description of motions, while the comparison between the pre and posttest measure the effectiveness of the proposed learning path in help students to overcome the highlighted learning problems. 


\title{
WORKSHOP VRL:
}

\author{
Remote Controlled Experiments for High School and Undergraduate Physics Students \\ Jan Mulder, Piet Blankert and Gerrit Kuik \\ Vrije Universiteit Amsterdam, The Netherlands
}

\begin{abstract}
Introduction
Remote controlled experiments may be considered over hands-on labs for several reasons, e.g. experiments can be conducted independent of time and place, and access to sophisticated equipment may become feasible (a strong argument for high schools). However, for remote controlled experiments students are restricted in making modifications to the setup, and hence is limited in the freedom to investigate physical phenomena.

We will present a selection of remote controlled experiments (laser remote sensing (LRS), positron emission tomography (PET), X-ray fluorescence, laser Doppler anemometry (LDA), Fresnel diffraction, synthesis of methyl orange in a micro reactor), which allow first and second year bachelor students a lot of freedom in terms of defining research questions, arranging the experimental setup and conducting a remote experiment. For high school students, using a simplified interface and by providing supporting educational materials, somewhat more structured versions of the remote experiments can be conducted, still guiding them towards inquiry based experiments.
\end{abstract}

\section{Concept and Implementation}

The remote controlled experiments have National Instruments interfaces and are programmed in LabVIEW. The experiments can be accessed from any web browser as long as Microsoft Silverlight is available. For some of the remote experiments additional simulation software is available (e.g. on 3D-imaging techniques of PET data). For all experiments supporting information is available through websites (wikis) and downloadable manuals (separate information for high school and undergraduate students).

The remote controlled experiments can be tested by the participants in the presentation/ workshop (maximum of 20 participants, target: international conference members).

\section{Objectives and Assessment}

The objectives for remote experiments do not differ from the objectives of hands-on experiments offered in our lab courses:

- we train students to design and carry out experiments,

- we introduce students to a variety of experimental techniques,

- we expose students to a number of research skills (among others data acquisition and data analysis),

- we provide a framework for reporting and presenting results. We do so through open inquiry based experiments.

In the case of bachelor students conducting remote experiments, their research question, the experiment design (work plan), and data analysis are discussed with their lab supervisor (as is the case for hands-on experiments). Students are assessed on their input in these discussions, and on their lab report. For high school students this task is the responsibility of their physics teachers. Teachers may request for advise on the students' assessment. 
PARALLEL SESSION SIM/VID:

\title{
SIMULATIONS IN PHYSICS TEACHING AND LEARNING
}

Creating Electronic Books for Computers and Tablets Using Easy Java/JavaScript Simulations, EjsS Modeling Tool Loo Kang Lawrence Wee Ministry of Education, Singapore

\author{
Ask the Simulation: \\ Challenging Questions and Visual Answers in Virtual Environments \\ Dorothy Langley ${ }^{1}$ and Rami Arieli ${ }^{2}$ \\ ${ }^{1}$ Holon Institute of Technology, Davidson Institute of Science Education, Israel \\ ${ }^{2}$ Weizmann Institute of Science, Davidson Institute of Science Education, Israel
}

\section{VIDEOS IN PHYSICS TEACHING AND LEARNING}

\author{
Theory Experiment Interplay with Video-Based Problems \\ in Experimental Physics Courses \\ Sebastian Gröber, Pascal Klein and Jochen Kuhn \\ University of Kaiserslautern, Germany
}

\author{
Interactive Video Vignettes \\ Robert B. Teese ${ }^{1}$, Priscilla W. Laws ${ }^{2}$ and Kathleen Koenig ${ }^{3}$ \\ ${ }^{1}$ Rochester Institute of Technology, USA, ${ }^{2}$ Dickinson College, USA, ${ }^{3}$ University of Cincinnati, USA
}




\author{
Creating Electronic Books for Computers and Tablets \\ Using Easy Java/JavaScript Simulations, EjsS Modeling Tool \\ Loo Kang Lawrence Wee \\ Ministry of Education, Singapore
}

\title{
Introduction and Theory
}

This paper shares my 2014 December journey (tools used, design principles derived and modeling pedagogy implemented) when creating electronic books-chapters (epub3 format) for computers and tablets using Easy Java/JavaScript Simulations, (old name EJS, new EjsS) Modeling Tool. The theory underpinning this work is grounded on learning by doing through dynamic and interactive simulation-models that can be more easily made sense of instead of the static nature of printed materials.

\section{Concept and Implementation}

I have been developing stand alone EJS and more recently EjsS computer models to allow anyone to run them on their Android tablets and iPad. After the Singapore Easy Java/JavaScript Simulations, EjsS Modeling Tool workshop organised by Francisco Esquembre and Wolfgang Christian 25-28 November 2014, I started combining related computer models with supporting texts and illustrations into a coherent chapter, a logical next step towards tighter support for teachers and students.

I have since spent 6 months developing prototypes electronic chapters on the topics of Simple Harmonic Motion and Gravity customized for the Singapore-Cambridge General Certificate of Education Advanced Level (A-level).

Objectives and Assessment

I aim to inspire more educators to create interactive and open educational resources for the benefit of all and network with like minded educators to add elements of assessment for learning, such as automated marking in future releases of the electronic books-chapters.

Prototypes are available via links here:

http://weelookang.blogspot.sg/2015/05/20th-international-conference-on.html, the EjsS Singapore Digital Library http://iwant2study.org/lookangejss/

http://iwant2study.org/lookangejss/epub3/20150625gravity.epub

http://iwant2study.org/lookangejss/epub3/20150625shm.epub

and Apple iBook Store, Google Play Book and Kindle Book. 


\author{
Ask the Simulation: \\ Challenging Questions and Visual Answers for Project-Targeted Learning \\ Dorothy Langley ${ }^{1}$ and Rami Arieli ${ }^{2}$ \\ ${ }^{1}$ Holon Institute of Technology, Davidson Institute of Science Education, Israel \\ ${ }^{2}$ Weizmann Institute of Science, Davidson Institute of Science Education, Israel
}

Simulations are cognitive technologies that can facilitate presenting, manipulating and exploring rule-based models (Pea, 1987). Simulations can be employed for teaching and learning physics in a variety of methods (e.g. Girdwidz, 2007). Simulations differ in the wealth of content knowledge and representation methods of the modeled subject, and the tools they offer. Rich environments stimulate questioning behaviour and offer ways of manipulating the environment and reaching answers. Asking questions is a prerequisite of natural learning, but in traditional school settings this role is often taken by those who already know (teachers, or text books). The current study focuses on the potential of rich simulation environments for sustaining the generation of physics questions, and providing tools for discovering and presenting answers in the context of a web-search activity for high school physics students participating in the "Physics \& Industry" regional class program.

The "Physics \& Industry" regional class program is a credit-carrying, 15 month, out-of-school framework fostering excellence and providing a link to science-based industries for high ability high school physics students. Participants design a working model dealing with a real world problem based on electro-optical principles (Langley, Eylon \& Arieli, 2010). Due to the diversity of student physics background and the need to emphasize technological application aspects, key topics have traditionally been reviewed by the instructor using presentations and demonstrations. However, low student attention and disappointing retention levels have motivated a new instructional approach, more in line with the need of students to be active, to proceed at their own pace and follow their chosen paths in class and later at home.

The activity was given the motivational title "Collecting information for the final project report". After searching for information sources about main concepts and principles, students were required to find topic-related simulations, to formulate 20 questions to which the simulation could provide answers and to present the answers visually as screen shots. Students worked for about 30 minutes, with the instructor providing advice and technical tips.

At present we discuss preliminary results from the Bending Light simulation (PhET ref link). Question trends:

concrete, quantitative vs. general, inquiry oriented

using familiar, basic concepts vs. new concepts

environment set up: simple vs. advanced

employing familiar tools vs. novel tools

relating to a single phenomenon vs. multiple phenomena

purpose: description, explanations, mechanism, design, relationships, verification, analyzing simulation behaviour

formulation: faulty, vague vs. precise

We offer an analysis of about 400 questions students formulated on Refraction and Waves and will summarize the learning gained through this process. 


\section{Theory Experiment Interplay with Video-Based Problems in Experimental Physics Courses \\ Sebastian Gröber, Pascal Klein and Jochen Kuhn \\ University of Kaiserslautern, Germany}

\section{Introduction and Theory}

Theory Experiment Interplay (TEI) is an essential method in physics research and a mandatory structure of lectures in calculus-based experimental physics courses. In contrast to lectures, the weekly attended recitation problems at universities are predominantly theoretical paper-and-pencil-problems. Therefore we developed a new problem format "Video-Based Problem (VBP)", which requires theoretical as well as experimental activities related to video experiments. Modelling of TEI in different forms for introductory physics courses on university level is the design principle and the inherent structure of VBPs.

We assume three effects of TEI in VBPs: (1) According to a simple linear problem solving model "Analyze problem $\rightarrow$ construct solutions $\rightarrow$ check results", TEI strengthens the last step, which is often neglected by students, as well as the whole problem solving process: Obviously deviations between theoretical and experimental results caused by students' errors in reasoning, calculation or measurement lead to a revision of the problem solving process. (2) Explanations of experimental results and of systematical deviations between theoretical and experimental results require qualitative reasoning. (3) The additional experimental activities in VBPs as well as TEI generates a more comprehensive view on physics and a higher disciplinary authenticity in comparison to traditional problems.

Overall, the outcome of TEI in VBP should be a better conceptual understanding of physics.

\section{Concept and Implementation}

Data gathering and analyzing in video experiments is possible with the well-known video motion analysis combined with sensor-based measurement of mechanical and non-mechanical quantities displayed in the video experiment. This enables us to represent an essential part of experimental physics content by VBPs. Our research about TEI prioritizes mechanics, but as TEI is independent from the content, our approach is also valid for subsequent experimental physics courses. Therefore a research-based implementation of VBPs in such courses is a realistic option.

\section{Objectives and Assessment}

In summer term 2015 we performed a pilot study about TEI in an introductory mechanics physics course (30 students). To proof characteristics of the instruction material we compared 20 traditional problems and 20 VBPs with respect to cognitive load and disciplinary authenticity. To identify the described effects we analyzed results based on questionnaires about TEI and qualitative reasoning. In the talk we present examples for TEI in VBPs and report on results of the pilot study. 


\author{
Interactive Video Vignettes \\ Robert B. Teese ${ }^{1}$, Priscilla W. Laws ${ }^{2}$ and Kathleen Koenig ${ }^{3}$ \\ ${ }^{1}$ Rochester Institute of Technology, USA, ${ }^{2}$ Dickinson College, USA, ${ }^{3}$ University of Cincinnati, USA
}

\begin{abstract}
Introduction and Theory
Interactive Video Vignettes (IVVs) are short Web-based assignments for introductory science students. Each vignette addresses a learning difficulty identified by Physics Education Research (PER). It combines the convenience of online video with the interactivity of an individual tutorial. Many vignettes take a student 10 minutes or less to complete, even though they often contain predictions, measurements and data analysis activities.

A typical vignette makes use of the classic elicit-confront-resolve (ECR) technique: It elicits a prediction from the user before the user can see the experimental result, then confronts the user with the experimental result, and then helps the user resolve any differences between them. The ECR technique has been used effectively in the design of many research-based curricular materials. In addition, vignettes may include video analysis and graphing activities that provide interactivity.
\end{abstract}

\title{
Concept and Implementation
}

Vignettes are implemented as web applications using HTML5 and JavaScript. They work on most desktops, laptops and tablet devices. To enable others to create vignettes, we also developed Vignette Studio, a Java application that runs on most desktop and laptop computers. Vignette Studio has a drag-and-drop interface, allowing vignettes to be constructed by dragging pages into place and then adding various elements, such as videos, images, or multiplechoice questions, to each page.

The Interactive Video Vignettes Project team created a set of sample vignettes (available at www.compadre.org/ivv). They illustrate various approaches (instructor-led presentations, "person-on-the-street" interviews, discussions between students and instructors, etc.). They are all based on outcomes of PER that will help student users learn. At the same time they will inform the future development of interactive web-based materials. Vignette Studio can also be downloaded from the website.

\section{Objectives and Assessment}

IVVs are designed to be effective teaching tools. We used two research tracks to investigate their usefulness. First, we investigated what incentives motivate students to complete vignettes outside of class. The most effective method was to assign a small amount of academic credit for completion. Second, we used the Force Concept Inventory (FCI) and other pre/ post-tests to study learning gains. We found varying amounts of gain for different vignettes. The most effective vignette is on Newton's Third Law, which had a normalized gain on a related FCI question of $70 \%$ for the treatment group compared to $46 \%$ for the control group. We have recently been analyzing each vignette in light of student learning gains to determine best practices in creating future vignettes. Factors being considered include vignette topic, length, use of the elicit-confront-resolve technique, and overall student engagement. 


\title{
INVITED SYMPOSIUM ASS:
}

\section{INCORPORATING MULTIMEDIA AND ICT IN PHYSICS EDUCATION: FOCUS ON LEARNING PATHS AND ASSESSMENT.}

Pieter Smeets (Chairperson), Cito, Institute of Educational Measurement Arnhem, Netherlands Henk Pol (Discussant), University of Twente, Netherlands

Multimedia and ICT have taken an important place in Physics education. Multimedia and ICT give opportunities to teach other things. The real life can be brought into the classroom. The student can be more interactive with the software. Calculations can be left over to the software, so there is more time for real thinking in Physics.

In the last thirty years huge effort is made to construct Simulations and Projects with Video Measurement and Modeling, that are used in the physics classroom all over the world.

The challenge we have now is to incorporated the Multimedia and ICT in the education. If not these beautiful applications are only used occasionally, to adorn the lessons with funny toys. In the Symposium we will focus on three aspects that can take care of the incorporation.

1. Assessment of ICT and Multimedia: Focus on the differences between Formative and Summative Assessment, illustrated with experiences examples

2. Designing a learning Path for all students between 12 and 17 years, and the role of Formative and Summative assessment.

3. Education of teachers in Physics and the assessment of their learning.

We think now is the moment to work on firm incorporation of Multimedia and ICT in Physics Education. The contributions in this symposium show their ideas about the three aspects.

In the discussion after the presentations we like to focus on the strategies to implement these ideas in a worldwide plan.

We hope to convince you of the importance of our ideas, and hope for cooperation to realize the plans, maybe in an international cooperation project.

\section{Assessment of ICT in the Physics Classroom: Formative and Summative Pieter Smeets \\ Cito, Institute of Educational Measurement Arnhem, The Netherlands}

\section{Roles and Ways of Assessment of Modelling and ICT-Supported Experimenting Competencies on a Modelling Learning Path in Secondary Physics Education in School Practice Onne van Buuren ${ }^{1,2}$, André Heck ${ }^{1}$ and Ton Ellermeijer ${ }^{3}$ \\ ${ }^{1}$ University of Amsterdam, Netherlands, ${ }^{2}$ Haags Montessori Lyceum, Netherlands ${ }^{3}$ Foundation CMA, Netherlands}

\author{
Teacher Education on the Use of ICT in Physics Education \\ and the Assessment of Teacher Learning \\ Trinh Ba Tran ${ }^{1}$, Ed van Den Berg ${ }^{2}$, Ton Ellermeijer ${ }^{3}$ and Jos Beishuizen ${ }^{1}$ \\ ${ }^{1}$ Vrije Universiteit Amsterdam, Netherlands, ${ }^{2}$ Free University, Netherlands, ${ }^{3} \mathrm{CMA}$, Netherlands
}

\author{
Using Educational Data Mining Algorithms \\ for Classifying High School Students of Physics \\ Daniel Sánchez Guzmán \\ Instituto Politecnico Nacional, Mexico
}




\title{
Assessment of ICT in the Physics Classroom: Formative and Summative Pieter Smeets \\ Cito, Institute of Educational Measurement Arnhem, The Netherlands
}

\begin{abstract}
Introduction and Theory
In the Netherlands we have a context based exam curriculum in which specific contexts have been prescribed since the $80 \mathrm{~s}$. So schoolbooks, and as a result of that, most of the Physics lessons are context based as well. Because of the principle: "test what you teach", all exam questions are context based too. With the help of ICT (data acquisition, video-analysis modelling, data-analysis or a simulation program) a lot of problems on several subjects are becoming more clear.

There is a lot of attention for computer education in the Dutch schools and in Physics especially. Teachers and students can use software like COACH for doing measurements on film fragments and are allowed to use Systematic as a programme for solving problems in physical computing.

To ensure that the ICT is well anchored in the education, it is important that the ICT is anchored in the assessment. During the process of learning Formative assessment is needed. The student has to get feedback on his work and his learning gains.

After the process of learning, Summative Assessment is needed to award the learning and the skills of the student. The more the student learned with ICT, the higher his marks.

The Formative Assessment is a responsibility of each school. It can be adapted to the student, the assignment, the Software and Hardware in school. The Summative Assessment is in the situation in Holland the same for every student. In the talk we shall focus on the similarity and the differences between Formative and Summative Assessment.
\end{abstract}

\section{Concept and Implementation}

There are two basic ideas about Assessment of ICT

1. The Assessment should be with the same software as the student uses in his learning.

2. The assessment should focus on the Physics and not on general or specific Computer Skills.

We show some examples of these ideas in the assessment we developed in the Compex project.

From 2003 to 2010 we have had the COMPEX-project in the Netherlands. For four subjects and in three levels in the central exam the pupils had to answer questions using software used in the Physics Class. The questions and answers were on paper. The purpose of the project was to assess the skills they learned in the Physics Lessons.

We constructed exams in which about one third of the questions should be answered using the software on the computer. The same software the pupils use in the classroom.

For three levels: lower vocational, higher vocational and pre-University exam we constructed exams where we tested mechanics. electricity, heat, radiation, informatics in Physics, optics. For the lowest level we adapted Coach to Coach-junior, for video-measure and modeling, but also Excel and we constructed our own applet-like applications for assessment purpose. In all the cases the results of the pupils were saved on the computer. This resulted in high levels of interactivity.

\section{Objectives and Assessment}

We shall focus on the similarity and the differences between Formative and Summative Assessment. In association with the two basic ideas we will give examples of our Assignments and will discuss with the participants if our goals are gained. 


\author{
Roles and Ways of Assessment of Modelling and ICT-Supported Experimenting Competencies \\ on a Modelling Learning Path in Secondary Physics Education in School Practice \\ Onne van Buuren ${ }^{1,2}$, André Heck ${ }^{1}$ and Ton Ellermeijer ${ }^{3}$ \\ ${ }^{1}$ University of Amsterdam, Netherlands, ${ }^{2}$ Haags Montessori Lyceum, Netherlands \\ ${ }^{3}$ Foundation CMA, Netherlands
}

\title{
Introduction
}

For both computer modelling and experimenting with ICT in physics, many competencies are required. To mention a few: modellers and experimentalists must be able to use software, analyze and interpret graphs, have sufficient understanding of the involved concepts and formulas, and modellers must understand their modelling approach. These competencies cannot be mastered in just a few lessons by a novice student but require a learning path distributed over a long period of time. Recently, such a learning path on computer modelling, combined with experimenting, has been developed for the Dutch lower secondary physics curriculum. This learning path is completely integrated into the curriculum and has been tested in school practice (Van Buuren, 2014). At the end of this learning path, students can build simple quantitative computer models themselves. Currently, this learning path is extended into the first year of upper secondary education.

The development of the students' competencies must be monitored carefully, by the teacher, but also by the students themselves. Several ways of assessment have been used to monitor the learning process, such as built-in possibilities for self-assessment, fast feedback techniques, questions in regular pen-and-paper tests, and practical assignments in which students do small size research projects. Their modelling and ICT-based experimenting competencies have been tested both with and without a computer at hand.

\section{Research Method and Implementation}

This study can be classified as educational design research. Educational materials have been designed, tested in classroom and redesigned in several cycles. Observations, audio recordings and screen recordings have been made of multiple student groups. Written materials and assessments have been collected. As an educational tool, we have used Coach 6 (Heck, Kedzierska, \& Ellermeijer, 2009), because in this computer learning environment modelling can be combined with doing and analyzing measurements. The graphical version of Forrester's system dynamics is used as a modelling approach. This approach has both advantages and limitations in school practice with respect to feedback and assessment.

\section{Objectives}

In the paper, we discuss the educational and practical advantages and limitations of these different ways of assessment, including the effect of assessment on students' attitudes and learning processes. Many modelling and experimental competencies can be effectively tested by means of regular pen-and-paper tests.

\section{References}

Heck, A., Kedzierska, E., \& Ellermeijer, T. (2009). Design and implementation of an integrated computer working environment for doing mathematics and science. Journal of Computers in Mathematics and Science Teaching, 28(2), 147-161.

Van Buuren, O. (2014). Development of a Modelling Learning Path (Doctoral thesis, University of Amsterdam). University of Amsterdam, Amsterdam. 


\author{
Teacher Education on the Use of ICT in Physics Education \\ and the Assessment of Teacher Learning \\ Trinh Ba Tran ${ }^{1}$, Ed van Den Berg ${ }^{2}$, Ton Ellermeijer ${ }^{3}$ and Jos Beishuizen ${ }^{1}$ \\ ${ }^{1}$ Vrije Universiteit Amsterdam, Netherlands, ${ }^{2}$ Free University, Netherlands, ${ }^{3} \mathrm{CMA}$, Netherlands
}

\begin{abstract}
Data logging with sensors, video measurement, and computer modelling are very relevant in physics education and have been available for thirty years. However, these tools are still very much "under-used". Factors involved are not only curriculum, assessment, available equipment and technical support, but also teacher education. Our study aims to contribute to the change in teacher preparation and training. We designed, taught, and evaluated a short training for science teachers to learn to integrate the ICT tools into Inquiry Based Science Education (IBSE). Central objectives of the training were that teachers master technical skills with ICT tools for experimenting or modelling in secondary-school science (first goal), and they are able to implement an ICT tool with inquiry-based methods in the classroom (second goal).

We developed and used a computer test to assess the first goal. The teachers took the test at the final course session. In 20 minutes, they had to improve a given data logging, video measurement, or modelling activity that was incomplete or that had something wrong. With this test, we measured teacher skills with an ICT tool at the level of problem solving.

In regard to the second goal, teachers were first required and supported to carry out the complete cycle of designing, trying out, and evaluating an inquiry-based activity with ICT. We collected lesson plans of teachers and observed their classroom try-outs of the ICT in IBSE activity. We used an analysis and coding schema to evaluate teachers' intended vs. actual ICT in IBSE lessons. The analysis and coding schema was adapted from the Laboratory Analysis Inventory - LAI by Tamir and Lunetta (1981) and from the Practical Activity Analysis Inventory - PAAI by Millar (2010).

In the symposium we will introduce the instruments to assess teacher learning in regard to the two goals of the ICT in IBSE training. Subsequently we will present and compare learning outcomes of pre- and in-service teachers the Netherlands, Slovakia, and Vietnam. Afterwards we will discuss how to improve the quality of these assessment instruments.
\end{abstract}

\title{
References
}

Millar, R. (2010). Analysing practical science activities to assess and improve their effectiveness: The Practical Activity Analysis Inventory (PAAI). Hatfield [England]: Association for Science Education.

Tamir, P. \& Lunetta, V.N. (1981). Inquiry-related tasks in high school science laboratory handbooks. Science Education, 65(5), 477-484. 


\author{
Using Educational Data Mining Algorithms \\ for Classifying High School Students of Physics \\ Daniel Sánchez Guzmán \\ Instituto Politecnico Nacional, Mexico
}

\title{
Introduction and Theory
}

Educational Data Mining (EDM) has evolved as a useful tool for analyzing and discovering of patters in the learning process. One of the main approaches is to classifying students as learning styles and skills that they can perform, also the evaluations can help us to understand how they perceive the instruction process. For researches these kind of tools can develop different learning scenarios based in an historical behavior, learning results and students interactions.

\section{Concept and Implementation}

When we apply a learning sequence we have the difficult to detect how learning is happening, one way is using basics statistics and on the other hand is using formulas proposed like the Hake's gain or the Concentration Factor proposed by Bao. In this work we present the use of the classifying algorithms in EDM to determine the best learning sequence for a group of students of Physics for the learning concepts of Ohm's Law, Voltage, Resistance and Current.

Objectives and Assessment

In this work we will show how the final evaluations and summative evidences can help us to determine a good learning sequence, based on different activities that we evaluate of the students and apply the classification algorithms. 


\title{
WORKSHOP MAP:
}

\author{
Creating Interactive, Online Concept Maps using Free Software \\ Ulaş Üstün ${ }^{1,2}$, Raiya Ebini ${ }^{1}$ and Dean Zollman ${ }^{1}$ \\ ${ }^{1}$ Kansas State University, USA, ${ }^{2}$ Artvin Çoruh University, Turkey
}

\begin{abstract}
Introduction and Theory
Recently, an online course for Contemporary Physics has been developed at Kansas State University. Different from many other online courses, this course offers students an interactive learning environment by means of many simulations, videos and online quizzes. The course has already been offered twice. Based on the feedback provided by the students enrolled in this course, we needed to integrate some advance organizers at the beginning of each module. As others have learned (See for example HyperPhyiscs at http://hyperphysics. phy-astr.gsu.edu/hbase/hframe.html) concept maps are a tool, which can be used as an effective advance organizer. We decided to develop one concept map for each module. In keeping with the general structure of the course, we preferred to make the concept maps interactive by adding hyperlinks to each concept within the concept maps. This process can be time consuming. However, some freely available software makes the effort much easier.
\end{abstract}

\section{Concept, Objectives and Implementation}

IHMC CmapTools (http://cmap.ihmc.us) is used to construct the concept maps. CmapTools provides us with the flexibility to create and modify general structure of the concept maps and to change many things related to format, the direction of the arrows etc. We make these maps interactive with the free program, CSS Image Map Generator (http://webpresencepartners. com/tools/css-image-map-generator/) to create hyperlinks within the concept maps. With this program, one can select any area within a graphics file and define a link for that area. In this application, we use JPG files which are generated with CmapTools as the graphics. We create a separate area for each of the concepts in the map and link each of these areas to an appropriate Web page in our lessons. When a user clicks within that area, he/she is taken to that link. The workshop participants will work with these two programs to create interactive concept maps.

\section{Assessment}

The workshop will provide an opportunity to construct concept maps and to make them interactive by using free software. The participants will also critique some of our concept maps and assess the value of these types of interactive maps in both our teaching and their own.

\section{Acknowledgements}

The development of the online course is supported by the KSU Global Campus. Dr. Üstün's participation is supported by The Scientific and Technological Research Council of Turkey (TÜBITTAK). 


\section{POSTER ABSTRACTS}




\author{
Comparing Two Learning Methodologies That Use Technology: \\ Intelligent Tutoring Systems Versus Interactive Simulations \\ Diana B. López Tavares ${ }^{1,2}$, Ricardo García-Salcedo ${ }^{2}$ and Daniel Sánchez Guzmán ${ }^{2}$ \\ ${ }^{1}$ Universidad La Salle Bajío, ${ }^{2}$ Instituto Politécnico Nacional, CICATA Legaria
}

\begin{abstract}
Introduction and Theory
Intelligent Tutoring Systems (ITS), have demonstrated that can improve the learning and skills in different age students; based on the concept of personalized learning, cognitive model of students, interaction between the tutor and student, feedback and capturing student interactions for future research; these systems can achieve a self-learning. On the other hand interactive simulation can help students to comprehend physical phenomena that can be difficult to see in a physical way. As we can combine both approaches it is important to analyze different learning scenarios.
\end{abstract}

\title{
Concept and Implementation
}

In present work we show the results of implementing two different learning sequences; both were running in a parallel way. The first learning sequence used active learning based on experiments in classroom, also it was combined with interactive simulations and at the end the students solved basic electric circuit exercises for finding current and resistance in electric circuits in their notebooks. For the second learning sequence students only faced lecture explanation, classic laboratory exercises, interactive simulations and intelligent tutoring systems for learning the same concepts. For analyzing results we used items from the CSEM. At the end we analyzed results comparing with Hake's gain and concentration factor.

Objectives and Assessment

We want to find which is the relation of using technology with high school students and how we can propone an effective learning sequence. In some cases technology has to be well situated inside a learning sequence to complement and obtain a long-term learning success. 


\author{
Integrating Simulations and Hands-On Activities in Physics \\ Pre-Service Teacher Education \\ Sabine Hemmer, Sandra Moretto and Ornella Pantano \\ University of Padova, Italy
}

\title{
Introduction and Theory
}

Research suggests that the use of simulations in science teaching enhances the motivation and performance of students (Rutten et al., 2012; Wieman et al., 2008). As part of a teacher training course we offered a laboratory on electricity integrating hands-on activities and simulations. While providing the pre-service teachers an opportunity to experiment and reflect upon this kind of learning environment, the laboratory allowed to study the effectiveness of the proposed educational activities in the context of teacher training.

\section{Concept and Implementation}

The study investigates the use of simulation and its interplay with hands-on activities for pre-service secondary physics teacher education. All 45 participants had a masters degree in physics or mathematics. A conceptual test on electricity was administered to study the participants' prior knowledge. Information about prior laboratory experiences was collected. The laboratory activities focused on DC circuits. The participants were divided into two groups: one started with hands-on exercises and subsequently performed simulations, the other one followed the reversed order. The tasks required numerical or qualitative predictions before simulating or building a certain circuit to check the answers with measurements. The participants were asked to reflect on the task-related difficulties, concepts, and surprising aspects.

\section{Objectives and Assessment}

Results from a preliminary analysis suggest that there were no differences in performance between the two groups. When using the simulations, making predictions based on prior knowledge did not present any difficulty and even though $98 \%$ of the participants had no prior experience with simulations no one encountered difficulties during their execution. This confirms that simulations reinforce students' understanding of relationships between variables by providing exact agreement with the predicted outcome (Sethi, 2005). On the contrary, in case of the hands-on activities including simple wires, batteries and bulbs, making predictions and measurements resulted very problematic for almost all participants. Both groups encountered an equal amount of difficulties. It seems that the simulations do not help the pre-service teachers in transferring their conceptual knowledge to the reality. A quantitative analysis of the results and a study regarding the pre-service teachers' attitude towards the activities is ongoing.

\section{References}

Rutten N. et al. (2012). The learning effects of computer simulations in science education. Computers \& Education, 58, 136-153.

Wieman C. E., Adams W. K. \& Perkins K. K. (2008). PhET: Simulations that enhance learning. Science, 322, $682-683$.

Sethi, R. J. (2005). Using virtual laboratories and online instruction to enhance physics education. Journal of Physics Teacher Education Online, 2(3), 22-26. 


\section{Teaching Quantum Physics: \\ A Review of Research on Misconceptions, Teaching Strategies and Use of Multimedia Kim Krijtenburg ${ }^{1}$, Henk Pol ${ }^{1}$ and Wouter van Joolingen ${ }^{2}$ \\ ${ }^{1}$ University of Twente, The Netherlands, ${ }^{2}$ University of Utrecht, The Netherlands}

\section{Introduction}

Quantum physics is the foundation of modern physics and therefore implemented in high school physics curricula across the world. As Quantum physics describes phenomena different from classical physics, research on understanding, teaching strategies and visualization tools is needed but still in its infancy. Generally three research topics are of interest:

- Understanding and misconceptions: Student understanding is investigated and tools and models are developed for better comprehension of misconceptions.

- Influence of curricula and teaching strategies: Teaching strategies and curricula are compared and evaluated for learning effects.

- Development of multimedia: Simulations are designed to provide visualization tools and interactive activities for better understanding.

Singh et al. (2006) pled for use of multimedia together with knowledge of misconceptions and a coherent curriculum, but few researchers thoroughly combine these aspects of educational research. McKagan et al. (2008) developed and used a simulation to uncover difficulties in student understanding of tunneling. Zhu and Singh (2012) developed, implemented and evaluated a research-based learning tool to improve understanding of quantum measurement.

\section{Method}

More exchange between the three research fields would benefit the development of a research-based approach for teaching quantum physics in high schools. To stimulate exchange, a literature review was conducted. 96 articles were selected and analyzed for investigated misconceptions, mental models, teaching strategies, research tools and multimedia applications. Outcomes were categorized for their contribution to the subtopics of quantum physics.

\section{Results}

This investigation gives an overview of misconceptions, mental models, teaching strategies, research tools and multimedia applications for quantum physics. It also pictures the (lack of) research-based multimedia for several subtopics and the need for multimedia addressing existing misconceptions. The overview presented in this research therefore cannot only be used as a starting point for further exchange of knowledge, it also outlines new opportunities for research and multimedia development.

\section{References}

McKagan, S., Perkins, K., \& Wieman, C. (2008). Deeper look at student learning of quantum mechanics: The case of tunneling. Physical Review Special Topics - Physics Education Research, 4(2), 020103.

Singh, C., Belloni, M., \& Christian, W. (2006). Improving students' understanding of quantum mechanics. Physics Today, 59(8), 43-49.

Zhu, G., \& Singh, C. (2012). Improving students' understanding of quantum measurement. II. Development of research-based learning tools. Physical Review Special Topics - Physics Education Research, 8(1), 010118. 


\author{
On the Double-Slit-Experiment; Applet Versus Demonstration \\ Henk Pol ${ }^{1}$, Kim Krijtenburg ${ }^{1}$, Aernout van Rossum ${ }^{1}$ and Wouter van Joolingen ${ }^{2}$ \\ ${ }^{1}$ University of Twente, The Netherlands, ${ }^{2}$ University of Utrecht, The Netherlands
}

\title{
Introduction and Theory
}

Recently, quantum physics was introduced as a new part of the Dutch physics curriculum for upper secondary education. The topic refers to phenomena in the real, visible world that can only be understood using quantum mechanical concepts.

The new domain includes wave-particle duality, the photoelectric effect, quantum effects, the uncertainty principle, and tunneling. Contexts discussed are i.e. digital camera, LED, colorants, microscopy, etc. The emphasis of the new topic is therefore on conceptual understanding rather than on the mathematical formalism of quantum mechanics (Akarsu, 2011; Taber, 2005).

\section{Concept and Implementation}

When introducing the subject of quantum physics, there is a need for practicals to support teaching quantum mechanics in a conceptual way. Practical experiments have the potential of supporting the acquisition of conceptual knowledge in a better way than simulations or applets (Abrahams \& Millar, 2008). Currently, the collection of conceptual practicals for quantum mechanics is small, including on the photoelectric effect and spectral lines. (Akar$\mathrm{su}, 2011$ ). This poster presents a project in which conceptual practicals for quantum physics are developed. The project focuses on the design of practicals that, supplemented by with available applets, are usable in conceptual lessons on quantum mechanics (Etkina, 2002). The poster presents the specific case of the double-slit-experiment for light in which individual photons are counted. In the experiment, results are made visible and audible with a Geiger counter.

\section{Objectives and Assessment}

The double-split experiment is used in teaching the principle of wave-particle dualism. The main question is whether a demonstration convinces the student of this concept, or that additional applets are needed. After an introduction of the double-slit experiment, many students still had problems in understanding the concept of wave-particle dualism. In a study we combine the demonstration with applets, before or after the experiment to improve student understanding. The poster will show first results of this study in the form of pre-post test data.

\section{References}

Abrahams, I., Millar, R. (2008). Does practical work really work? A study of the effectiveness of practical work as a teaching and learning method in school science. IJSE, 30, 1945-1969.

Akarsu, B. (2011). Instructional designs in quantum physics: A critical review of research. Asian Journal of Applied Sciences, 4, 112-118.

Etkina, E., Van Heuvelen, A., Brookes, D.T., Mills, D. (2002). Role of Experiments in Physics Instruction - A Process Approach. Phys. Teach. 40, 351-355.

Taber, K.S. (2005). Learning quanta: Barriers to stimulating transitions in student understanding of orbital ideas. Science Education, 89, 94-116. 


\title{
Towards Attractive Interactive Instructions for Practical Physics Laboratories Leonard Büsch and Heidrun Heinke RWTH Aachen, Germany
}

\begin{abstract}
The presented studies aim to develop and apply attractive interactive multimedia-based instructions for physics laboratories in order to exploit the advantages of technology enhanced learning in today's students' digital environment.

According to the JIM-study, there has been a dramatic growth during the last few years in the availability of PCs and laptops (99\%), tablet-PCs (48\%), as well as smartphones (94\%) for young people between the ages of 12 and 19 in Germany (numbers in brackets show data for 2014). The Kaiser Family Foundation claims that young Americans spent over 7 hours per day with digital media in 2010. Thus, requirements are met for the broad integration of technology enhanced learning concepts in students' everyday learning activities at university. For this reason, technology enhanced instructions for the physics laboratory will be offered online, so students can prepare interactively whenever and wherever possible for the handson laboratory. The laboratory in question is a physics laboratory, which mechanical engineering students $(\mathrm{n} \approx 1000)$ usually complete in their third semester. The technology enhanced instructions retain the same content as the paper version which has been used hitherto but are further expanded upon through videos to motivate the experiment and explain the physical background, simulations to improve understanding of theory and Interactive Screen Experiments to visualize the experimental set-up and to work through the first steps of the experiment. Finally, there is an opportunity for students to check their own knowledge.

These interactive elements were chosen as a consequence of a survey conducted with 125 students, in which over $75 \%$ of the students ranked the following as potentially helpful:

- Interactive Screen Experiments

- Simulations

- Videos about the physical background

- Videos about the performance of the experiment

- Possibilities for students to check their knowledge

- Questions for attendance taking

These elements are implemented into the technology enhanced instruction based on knowledge of the students' approach to the real experiment. For this, the students' performance during the experiment was analyzed in detail utilizing Smartpen data. These pens can record communication among students while they are working in pairs on the experiment and while discussing their experimental progress and problems. Simultaneously, their notes are also recorded, which creates a big data pool for the design of the interactive elements mentioned above and allows us to pinpoint problem areas to be addressed by the experiment instructions.

In this contribution the results of user-orientated process of designing and optimizing technology enhanced laboratory instructions and their application by students will be presented exemplarily for an experiment on radioactivity.
\end{abstract}


Poster Session

Contribution P06
Room P (F 104 / Fakultätensitzungszimmer)

Wed, 9 Sep, 16:30-18:30

\author{
Using Videos to Develop an Understanding of Measurement Uncertainties \\ John Hamacher, Jan Erkelenz and Heidrun Heinke \\ RWTH Aachen, Germany
}

\begin{abstract}
An important goal of physics laboratories is to enable students to develop experimental competencies. In established models of experimental competence data analysis is a prevalent phase. National and international studies indicate that especially the data analysis is a crucial and important task where many students encounter difficulties and don't reach the level of competence aimed for. The most serious problems within the broad field of data analysis are the correct understanding and calculation of measurement uncertainties. A detailed look at the process of writing protocols in an introductory physics laboratory reveals that the students spend a large amount of time (on average seven hours per protocol) to carry out the analysis. While they are working on their protocols at home, students typically are not able to request for direct help from a tutor. Thus, there exists a fundamental structural problem in typical physics laboratories: Students are at home confronted with the hard felt and time consuming task to analyze their experimental data and deal with the difficult problem to discuss and quantify measurement uncertainties without appropriate support.

A new approach to convey the handling of measurement uncertainties in the introductory physics laboratory is under development at RWTH Aachen University and will be applied first in physics lab for biology and biotechnology students. To tackle the mentioned structural problem it was decided to create a series of online videos, which explain and demonstrate the correct understanding and calculation of measurement uncertainties specifically for this target group. This decision was based on our own preliminary work where Effertz et al. investigated the influence of a first generation set of videos on student's ability to complete easy tasks of data analyses correctly. It was shown that students performed significantly better if they were allowed to use the video tutorials. The student's reaction to the videos was throughout positive and they indicated that videos would be reasonable and useful to support their learning processes in the physics lab. Based on these results, second generation videos were produced. They were in the focus of a usability-study, the results of which will be presented. Participants of this study were requested to use the think-aloud method while solving simple tasks concerning measurement uncertainties. Via smartpen and screencapturing- software it was possible to record everything they said, the development of their solution and their clicking-through behavior in parallel. Based on the results of this study, the concept for the series of online video tutorials on data analysis in an introductory physics lab was redesigned which will be discussed in the paper. Moreover, the videos will be embedded in a new overall concept of handling measurement uncertainties in introductory physics laboratories at RWTH Aachen which will be presented.
\end{abstract}


Poster Session

Contribution P07
Room P (F 104 / Fakultätensitzungszimmer)

Wed, 9 Sep, 16:30-18:30

\section{Seeing How Fitting Process Works \\ Vera Montalbano and Mauro Sirigu \\ University of Siena, Italy}

\section{Introduction and Theory}

In order to engage students in understanding the physical world by constructing and using scientific models to describe, to explain, to predict, to design and control physical phenomena, a central role is played by the fit of data measured in a physics experiment. For developing insight into the structure of scientific knowledge by examining how models fit into theories and for showing how scientific knowledge is validated, students must be engaged in evaluating scientific models through comparison with empirical data (Hestenes 1997). The concept of best fit of a sample of data, how the parameters in the proposed function change in order to obtain the best fit, the uncertainties related to these parameters are difficult to explain to high school students because of their lacking of advanced mathematical tools. Moreover, many attempts of showing examples can be too abstract or remains obscure because of using of software which does not show in an explicit way the fitting process.

\section{Concept and Implementation}

A very effective and active way of teaching how a fitting process works emerged in a course focused on physics lab didactics in pre-service education of physics teachers. The proposal was designed during the data analysis of an experiment on the RC circuit. A picture of the oscilloscope display with the charge and discharge of the capacitor was imported in GeoGebra and a visual realization of the fitting process was explored.

\section{Objectives and Assessment}

Some teaching/learning path focused on the comparison between predictions from a model and empirical data realizable in high school were designed in order to test in classroom, as soon as possible, the effectiveness of the active visualization of the fitting process.

\section{References}

Hestenes, D. (1997). Modeling methodology for physics teachers. In AIP CONFERENCE PROCEEDINGS (pp. 935-958). IOP INSTITUTE OF PHYSICS PUBLISHING LTD. 


\section{Learning Particle Physics Using Timepix-Based Pixel Detectors at CERN S'Cool LAB Julia Woithe, Konrad Jende, Oliver Keller and Sascha Schmeling CERN, Switzerland}

\section{Introduction and Theory}

$S^{\prime}$ Cool LAB is an international out-of-school hands-on laboratory for school students at CERN, Geneva, Switzerland. During workshops in S'Cool LAB, students work with high-tech equipment to independently perform modern physics experiments that are linked to CERN's technologies and physics [1]. By doing so, students gain insight into the world's largest particle physics laboratory. Among S'Cool LAB's equipment is a special pixel detector, the MX-10 particle camera, which uses the Timepix chip [2] developed by the Medipix2 Collaboration at CERN [3] and has been re-purposed for school students by JABLOTRON ALARMS [4].

\section{Concept and Implementation}

The range of potential research topics for the MX-10 pixel detector includes:

Background radiation: Students can study tracks of particles originating from space or naturally occurring radioactive isotopes in real time. They distinguish different types of particles by their specific signature recorded in the detector.

Properties of ionizing radiation: Students can use the detector to verify the inverse square law, study the attenuation of ionizing radiation in matter, determine emission spectra of radioactive sources and examine unknown radioactive materials.

X-Ray imaging: Combining the MX-10 detector with an X-Ray source, students can use the detector as online particle camera. Objects like insects or memory cards can be X-rayed, the different absorption of photons in the material resulting in a radiograph.

\section{Objectives and Assessment}

The goal of S'Cool LAB is to integrate the MX-10 pixel detector and its capabilities into handson particle physics workshops. Worksheets guide students through the different experiments taking into account their prior conceptions related to radiation. These worksheets are constantly being retested and redesigned within the framework of design-based research [5]. As well as the description of experiments implemented, the process of student worksheet development will be presented.

\section{References}

[1] Homepage of CERN S'Cool LAB: http://cern.ch/s-cool-lab (retrieved 26/05/15)

[2] X. Llopart, R. Ballabriga, M. Campbell, L. Tlustos, W. Wong (2007). Timepix, a 65k programmable pixel readout chip for arrival time, energy and/or photon counting measurements. Nuclear Instruments and Methods in Physics Research Section A 581 (1-2) 485-494

[3] Homepage of Medipix Collaboration: http://medipix.web.cern.ch/medipix/index.php (retrieved 26/05/15)

[4] Homepage of MX-10 Digital Particle Camera: http://www.particlecamera.com/index.php (retrieved 26/05/15)

[5] Design-Based Research Collective (2003). Design-based Research: an emerging paradigm for educational inquiry. Educational Researcher, 32, 5-8 


\author{
Analysis of Didactic Material of Undergraduate Physics Course \\ of Ceará Federal University in the Modality Of Distance Education \\ Caniggia Pereira and Eloneid Nobre \\ Federal University of Ceara, Brazil
}

\begin{abstract}
In today's world, where Information and Communication Technologies (ICT) are in continuous development, the distance learning is becoming an increasingly strong trend. All over the world, the online study has been an excellent way to bring the University to the student's home, allowing a high degree of flexibility in any academic program. There is no doubt that the multimedia technology is a powerful tool in the development of e-learning contents, which is inserting deep changes at higher education [BAGGALEY, Jon; KIRKUP, Gill]. According Peterson and Palmer (2011), students of distance learning courses that use online platforms tend to have higher levels of technological skills. However, the preparation of teaching materials requires special care. It is necessary that the institutional routines are designed and rebuilt in a continuous process of organizational learning, which consider the interrelationships between the processes and actions (Cazarini et al., 2012).

This study aims to analyze the teaching materials used in the Undergraduate Physics Course in the distance modality, offered by the Federal University of Ceara. We chose to analyze the disciplines Physics I, II, III and IV and Topics of Modern Physics. Besides these, we decided also to examine the didactical materials of Mathematics I and II. These disciplines are offered at the beginning of the course. They are prerequisites for students can attend the disciplines of Physics, and in general, are also those with large failure rates. From the analysis of the teaching material we apply a questionnaire involving items covering the teaching resources and didactic transition, analyzing the contributions of each resource for understanding the content and learning. The questionnaire, based on the Likert scale, will be administered online through the platform used for the development of distance learning courses at the University. We intend to build a picture that describes the didactic material Undergraduate Physics Course in the Distance modality, both from a technical point of view, and the opinion of the students themselves. From this information will be created a guide with suggestions for working with teaching materials for distance learning courses to ensure a better use of available resources for the realization of didactic handover of the material.
\end{abstract}

\title{
References
}

BAGGALEY, Jon; HOON, M., Ng Lee (2005) Pandora's Box: Distance Learning Technologies in Asia, Learning, Media \&amp; Technology, v30, (1).

CAZARINI, Edson Walmir; NETO, José Dutra de Oliveira; OLIVEIRA, Selma Regina Martins; TIZIOTTO, Simone Aparecida. Reflexões sobre a inovação na educação e distância: o caso brasileiro. EaD Em Foco, no. 02, p 94-103, 2012.

PETERSON, Sharon L.; PALMER, Louann Bierlein. Technology Confidence, Competence and Problem Solving Strategies: Differences within Online and Face-to-Face Formats. Journal of Distance Education, vol. 25, no 2, 2011. 


\title{
Use of QR-Codes to Provide Information and Assist Learning in Lab Courses Lars-Jochen Thoms, Andrea Lisa Nagel and Raimund Girwidz LMU Munich, Germany
}

\begin{abstract}
Introduction and Theory
Students of physics for higher education at the Ludwigs-Maximilians-Universität München visit two seminars with the topic of school relevant experimenting where they set up experiments on their own (in addition to the traditional physics education lab courses). In spite of the fact that data sheets and manuals of the lab equipment are available and we explicitly ask the students to read the material before setting up an experiment, students do not always operate the equipment appropriate. In the worst case, damages caused by faulty operation may happen.

In an exploratory interview study students proclaimed the lack of knowledge concerning the appropriate operation of the lab equipment as the main reason for a failure in experimenting. Due to lack of time during the preparation students did not read the data sheets and manuals in an sufficient way.
\end{abstract}

\section{Concept and Implementation}

To help students to get access to data sheets and manuals and to motivate them for a thorough inspection, we have digitalized the data sheets and manuals and have attached QR codes to the lab equipment. By scanning these codes with a smart phone or a tablet students receive data sheets, manuals, description of experiments, video manuals, and further device-specific information via the Internet. We may also include links to the a web-based team collaboration platform we use as learning platform at the LMU. In addition, we present a photograph of the device and inventory information.

Objectives and Assessment

To examine how this new proposition of information is accepted by students, we analyzed the consumption in the summer term 2014. Furthermore, we gave out six experimental exercises to students with printed QR codes referencing to equipment needed to conduct the respective experiment.

We paid special attention to the question in which phase of the experimental processing, regarding to experimental competencies, students use the QR codes to gain information. 


\author{
Design and Practice of an In-Classroom Moodle System Supporting \\ for Self-Teaching in the Elementary Physics Education in University \\ Yuichi Anada \\ Hokkaido Information University, Japan
}

\title{
Introduction and Theory
}

The percentage of students enrolling in universities has increased gradually and it reached more than $50 \%$ in Japan. Recently, a lot of Japanese universities take on students who do not have a sufficient basic level of academic ability. Regarding the education of physics, not a few students are a lack of basic concept and basic knowledge of physics. Active learning is useful method for engaging students to learning. However, the rudimentary knowledge of basic physical quantities, units of them and so on is prior condition to the active learning. An original review-test system for elementary physics in classroom has been developed in our university in order to fix the basic knowledge. In this study, an effect of the review-test on academic ability of physics was examined in our university.

\section{Concept and Implementation}

Students forget easily the knowledge acquired in the next class, even if an able teacher succeeded for the students to understand and to fix the knowledge. The concept of the reviewtest system is to revive the knowledge before starting the classwork of a few days later class. The review test is implemented in learning management system, Moodle. The course investigated in this study is an elementary physics course offered for second-year students. This course is optional and offers a lecture once a week throughout one university year. The total number of students who chose this course is approximately 100 . The review test is composed of two parts: one is a part of formulation of a concept of physical phenomena and another is a part of understanding of equations and symbols. During the review test, students have to refer to the text, their notes and other reference materials. It takes approximately $15 \mathrm{~min}$. Students is able to check a result of making for each question immediately by using the function of Moodle. The review-test system has not only the review-test but also a function of writing down students' reflection just after checking their mark.

\section{Objectives and Assessment}

As a result of several years' trial, the effect has verified in the term examination especially in the review test. Additionally, the effect was examined by pre-test and post-test for basic knowledge held at the beginning and the end of the term of this course. Furthermore, the effect was also examined by Force Concept Inventory (FCI). It was confirmed that the basic knowledge was fixed sufficiently. On the other hand, acquirement of the force concept is improved but not sufficiently. The effect of the reflection for the result of the review-test was also assessed by the result of two groups with and without reflection. The result of the post-test was improved in the group with reflection more than the group without reflection. Therefore, it is considered that the review-test system implemented in the Moodle is useful to fix the basic knowledge of elementary physics. 
Poster Session

Room P (F 104 / Fakultätensitzungszimmer)

Contribution P12

Wed, 9 Sep, 16:30-18:30

\author{
Blended Learning With Virtual Laboratories \\ in the Context of Application Oriented Education in Physics \\ Tobias Roth, Thomas Andres, Alexander Schwingel, Julia Appel and Ulla Hein \\ Trier University of Applied Sciences, Germany
}

\title{
Introduction and Theory
}

Practical courses play an important role for the quality of tertiary education in natural and engineering sciences, particularly with regard to the shape of advanced skills and special competences[1].

Therefore, the joint project Open MINT Labs (OML) - funded by the German Ministry of Education and Research (BMBF) - among Rhineland-Palatinate's universities of applied sciences Kaiserslautern, Koblenz and Trier puts the development and scientific investigation of virtual laboratories in the center of interest.

\section{Concept and Implementation}

Every virtual lab follows a modular concept, as elaborated by the project team, whereas taking into account recommendations from didactics of learning and media design as well. The structure consists of the following five self-explanatory bricks: Orientation, Basic Information, Experiment, Application and Reflection, that coach the learner from the buildup of basics to the transfer of knowledge when answering questions from everyday life or in order to solve challenging problems from research and industry with project character. The potential of interactive learning with multimedia are exploited, e.g. methods of direct feedback, tools of self-evaluation, while the virtual lesson encourages learners to work independently.

So to speak a unique selling point of OML is the cooperation with partners from industry to link the teaching and learning content with the student's perspective, and to illustrate the relevance for their later working life. Authentic interventions stimulate the learner's activity: The task may be to analyze the video of a physical or technical process, to perform a parameter study by applying numeric simulations or to examine a realistic three dimensional animation with CAD (computer aided design).

The virtual labs are implemented in the widespread learning management system OpenOLAT.

\section{Objectives and Assessment}

We show the concrete realization of virtual labs in use, and demonstrate how one can succeed to motivate students in spite of an abstract theoretical background. This flexible instrument of learning shall help undergraduates to prepare or to reinforce experiments in the real laboratory - irrespective of place, time and used end device.

In line with the philosophy of blended learning, virtual elements can be easily embedded in traditional formats like lectures, exercises or tutorials. The main goal is to establish virtual labs as an inherent part throughout the teaching program and therewith ensure sustainability. On top of that, the project OML is accompanied by a long-term empirical evaluation whose results help to understand the learning process and to continuously optimize the virtual labs.

\section{References}

1. Zwickl et al., Am. J. Phys. 81 (1) 2013, 63-70 


\section{Preparing Teachers to Use Educational Games, Virtual Experiments, and Interactive Science Simulations for Engaging Students in the Practices of Science Christian Fischer and R. Charles Dershimer University of Michigan, USA}

\section{Introduction and Theory}

Current educational reforms in the United States, initiated through the National Research Council's Framework for K-12 Education (NRC, 2012) and the Next Generation Science Standards (NGSS Lead States, 2013), promote changes in science education emphasizing 'inquirybased' instruction engaging students in science and engineering practices. While traditional forms of instruction might not be sufficient for effectively helping teachers with responding to these curricular changes, digital technologies provide promising tools for teachers to support student learning (e.g., U.S. Department of Education, 2010, 2013). Although it is tempting to overestimate the potential of digital technologies as an influence on student learning, materials and technology are necessary but not sufficient aspects of teachers' instructional capacity, as indicated in the instructional triangle (Ball \& Cohen, 1999). Therefore, we designed a workshop on how digital technologies can act as content resources, how to plan technology-enhanced instruction adopting reform-based standards, and how to integrate these tools in science teaching.

\section{Concept and Implementation}

This technology workshop was implemented in the Teaching Secondary School Science course for pre-service science teaching interns at the University of Michigan. Goals of the workshop are two-fold. First, exposing interns to various digital technologies and discussing implementation affordances in reform-based science instruction. Second, introducing a technology adoption framework to provide a scaffolding of practices for effective lesson planning with digital technologies. Digital technologies presented are educational games from BrainPOP's GameUp collection (BrainPOP, n.d.), virtual experiments from LMU Munich's Remotely Controlled Laboratory project (Girwidz, Pickl, \& Krug, n.d.), and interactive science simulations from UC Boulder's PhET Interactive Simulation project (University of Colorado, n.d.). Interns actively explored each tool through scaffolds for teaching preparation practices including strategies for selecting appropriate tools, designing new instructional materials, and aligning existing materials with teaching objectives.

\section{Objectives and Assessment}

As part of the workshop, interns were asked to reflect if, how, and why they might implement digital technologies in their own teaching. Reviews of these reflections in relation to the workshop goals indicate the benefits of integrating descriptions of tools with scaffolds for teaching preparation practices. For instance, interns' comments indicated they could justify their choice of tools for future classroom use based on how the features met their instructional goals. This workshop indicates that when preparing teachers to use digital technologies it is important to not only focus on technological aspects but also to provide guidance on how teaching can be aligned with teaching objectives and individual classroom contexts. 


\author{
Multimedia Content for Introductory Physics for Pre-Health Students \\ Ralf Widenhorn ${ }^{1}$, Ellynne Kutschera ${ }^{1}$, Elliot Mylott ${ }^{1}$, Justin C. Dunlap ${ }^{1}$ and Warren Christensen ${ }^{2}$ \\ ${ }^{1}$ Portland State University, Portland, OR, USA, ${ }^{2}$ North Dakota State University, Fargo, ND, USA
}

\begin{abstract}
Introduction
In the USA there is growing concern among experts in the physics and medical communities that undergraduate physics courses are not adequately preparing students for careers in medicine [1,2]. The rapid development of medical science has made it increasingly necessary that students entering the medical field possess a strong conceptual understanding of the relevant physical principles behind diagnostic and treatment technologies. This can be accomplished by engaging students in a course that is relevant to their future in the medical field. We present the design and assessment of a multimedia, flipped classroom, algebra based introductory physics course at Portland State University. The course utilized in class discussions, original texts, an online learning platform, content relevant videos from medical professionals, and laboratory sessions exploring the physics of medical instruments.
\end{abstract}

\title{
Implementation
}

The ten week course was designed to cover similar material to what is usually taught in the third term of a yearlong general physics class, specifically waves and optics. These topics were presented by highlighting their application to medical science and technology, such as ultrasound imaging, endoscopic surgery, microscopy, LASIK surgery, and pulse-oximetry. Before the class session, students were introduced to these topics through video interviews with biomedical professionals, who demonstrated the relevance of the content to the health field. Students then completed questions and activities about the videos and related physics content. This was done through questions and interactive content hosted on the smartFramework (sF) [3]. Student answers to the $\mathrm{sF}$ content were used by the instructor to structure the in-class lecture and discussion session covering the content in the video. This course was combined with a mandatory laboratory session, in which students explored the physics related principles of medical devices through original activities including a simulated pulse-oximeter [4].

\section{Assessment}

The effect of the course was probed using a 24 question online survey and the Colorado Learning Attitudes about Science Survey (CLASS) [5]. The 24 question survey was designed to measure students' satisfaction with the class as well as their attitudes toward specific aspects of the course. Student responses indicated that the video interviews with experts did help them to see the relevance of physics to the biomedical field and that this focus was an improvement over the previous course structure. Students were also positive about a number of elements used in the course including the online learning materials. Results from the CLASS data show trends toward more expert like responses in a number of categories. These results indicate that students responded positively toward a course designed to emphasize the relevance of physics to biomedicine.

This work was supported by grants (DUE-1141078 and DUE-1431447) from the National Science Foundation. 1. AAMC/HHMI (2009). Scientific Foundations for Future Physicians: Report of the AAMC-HHMI Committee.

2. Meredith, D. C., \& Redish, E. F. (2013). Reinventing physics for life-sciences majors. Physics Today, 66(7), 38.

3. https://www.flipitphysics.com/Account/Log0n?ReturnUrl=\%2f

4. Christensen, W., Johnson,J.K., Van Ness, G. R., Mylott, E., Dunlap,J.C., Anderson, E. A., \& Widenhorn, R. (2013). Developing and assessing curriculum on the physics of medical instruments. CBE-Life Sciences Education, 12(2), 250-261.

5 Adams, W. K., Perkins, K. K., Podolefsky, N. S., Dubson, M., Finkelstein, N. D., \& Wieman, C. E. (2006). New instrument for measuring student beliefs about physics and learning physics: The Colorado Learning Attitudes about Science Survey. Physical Review Special Topics-Physics Education Research, 2(1), 010101. 


\author{
A Distance Learning Course of Thermophysics \\ for Students From the Humanities and Social Masses \\ Xi Xia Liang \\ Inner Mongolia University, China
}

\title{
Introduction
}

Physics is the science that studies the nature and the universe. Its basic principles and methods have been applying not only in the modern technology, but also in the economics, finance, management and other fields. Needless to say, it is indispensable for students from the humanities to know summarily the basic principles and methods of Physics. Physical open online class is a good way to achieve this kind of Education. However, humanities students usually lose interest in Physics and believe that physical concepts are too difficult to be understood. Therefore, it is very important to arouse their interest by fusing scientific culture and humanistic culture and making the course easy to learn.

\section{Methodology and Implementation}

We have developed an open online course of thermo-physics for students from the humanities and social masses. The entire journey video recording of the class is online as a "Chinese Quality University Video Open Class" on "icourse" and "open.163", as well as "cntv" [1]. To arouse interest of humanities students in recognizing the physical world and help them understand physical concepts, we have taken the following measures in the class:

1. Permeating the elements of Chinese traditional culture in the course [2] to lead humanities students into the physics world;

2. Introducing Eastern and Western, ancient and modern experiences and stories on heat to help students understand the relevant thermo-physics laws and concepts;

3. Inditing classical poems explaining the physics contents to waken student's learning interest and help them understand and remember physics laws;

4. Taking full advantage of multimedia courseware and making simple animated drawings to demonstrate the physical phenomena and laws.

The online course attracts the liberal arts and science students, as well as social masses, and received good results. More than twelve thousand audiences have studied the course online. The Learners feel the course is vivid and easy to learn, even for the humanities students.

\section{Conclusions}

In summary our practices indicate that teaching open online science courses by fusing scientific culture and humanistic culture is necessary and feasible. It helps to arouse interest of students and make the physical concepts easy to understand.

This work is supported by the Chinese Quality University Video Open Class Fund, Ministry of Education of P. R. China.

\section{References}

[1] Liang, X. X., (2012). Excellent Reason in Heat. Video open course of China. http://www.icourses.cn/viewVCourse.action?courseCode=10126V002, http://v.163.com/special/cuvocw/rehanmiaoli.html, http://opencla.cntv.cn/china/jpk/20121029/VIDE100141154394.shtml.

[2] Liang, X. X., (2013). Permeating the elements of Chinese traditional culture into the physical classroom, China University Teaching, 2013(10), 7-9. 


\author{
A Suite of Computer Programs for Modeling Physics Problems \\ Stephan Lück \\ University of Würzburg, Germany
}

\begin{abstract}
Introduction and Theory
Many students have great problems in modeling even simple and uncomplicated physics systems or problems adequately. One reason is the often complicated mathematical solution of the modeling equations. In the first ages of the secondary school, the students are not even able to solve the equations at all. Therefore a tool is needed to overcome this obstacle. Ideally such a tool has to be as simple as possible to use so the students can concentrate their minds on the modeling process and the results of the solution. One can learn many aspects of physics during the process of modeling the examined topic, varying parameters, analyzing the results and comparing them with reality.
\end{abstract}

\title{
Concept and Implementation
}

As stated in the introduction it is very important to support the students during the modeling process adequately. In this contribution a suite of computer programs is presented to achieve this goal. The key aspects during the design of the programs are: great usability, direct feedback and robustness so the modeling and learning process does not stock because of unnecessary annoying and peripheral difficulties which distract the students from the learning subject.

The suite of java based programs consists of three computer programs which look much alike and are similar to use. The first application is called "Newton-II" and as the name tells, it is a solution tool for the second law of Newton. To model a mechanical problem a student only has to specify the acceleration via the forces on a particle of mass, specify the values for the parameters and the initial conditions and then run the solution via a simple "play button". The results appear immediately beside the modeling equation and values so that the students do not loose the connection of both. The second application is called "Fluxion" and can be regarded as the big brother of "Newton-II“. With the same easy-to-use-GUI the student is able to model and calculate any physical problem which can be described with a set of ordinary differential equation. So "Fluxion" is universal and not limited to mechanical problems. The third application is named "Lagrange“. The aim of this program is to model conservative physical systems while specifying the potential and kinetic energies. This is very helpful for a more deeply understanding of the influence of the potential energy on the resulting motion. Otherwise while modeling the same topic with "Newton-II" and "Lagrange“, one can elucidate the equivalence of a force based and an energy based description of a system.

\section{Objectives and Assessment}

The objective of the presented poster is to show the three applications of the suite in detail. Several purposes of use will be described and the programs will be shown on a laptop computer during the poster session. Furthermore, experiences and the assessment of several years of usage of the three programs in secondary schools and university will be shown. 


\author{
Digital Lab Manuals in Teaching-Learning-Labs \\ Frank Finkenberg and Thomas Trefzger \\ University of Würzburg, Germany
}

\begin{abstract}
Introduction and Theory
Digital lab manuals (DLM) are used in teaching-learning-labs at the University of Würzburg. In compulsory lecture courses, pre-service teachers of science arrange experimental stations which are connected to specific modules based on the physics school curriculum. Furthermore, the pre-service teachers create PDF-files as DLM for tablets. When school classes come to perform the lab, tablets are handed to the students who independently carry out the experiments under supervision of the pre-service teachers.
\end{abstract}

\title{
Concept and Implementation
}

The tablets serve as guides, manuals, record books and work books for the students. Based on the tablet hardware, DLM provide informational elements such as graphical and video instructions, text boxes, pop-up notes, and editable elements like drop-down lists, multiple choice menus, diagram drawing fields and free text boxes. The concept of DLM is to simplify the workflow of the students. They easily grasp the step-by-step instruction for each experiment by swiping through photo based and text restrained slides. Students can better retrace the arrangement of an experiment because they can stop or leap back in an explanatory video clip at any time. The editable elements featured by PDF help efficiently to secure the experimental results and to expand the range of possibilities for consolidating new knowledge.

Another aspect of implementing DLM is to better reach the students. Even though the experiments are designed to catch the student's interest, experience shows that students have problems to stay focused after reading and working through many pages of paper manuals. DLM try to maintain the interest by various channels of input, graphical underpinning, and different ways of securing results. Last but not least, the usage of DLM avoids a huge amount of paper to be copied and contributes thus to a more efficient way of setting up a teachinglearning-lab.

\section{Objectives and Assessment}

After a few cycles of teaching-learning-lab courses an empirical assessment of the DLM is still pending. However, first observations show that the motivational benefit of using DLM is undeniable. The students visibly enjoy working with the tablets and are eager to fulfill the interactive tasks. The instruction time for each experiment is shortened due to video tutorials and graphical reductions. Roughly speaking, DLM sustain the interest and motivation of the students for a longer time than traditional paper manuals do and are thus more effective. Two problems arise by using DLM in practice. Firstly, the students cannot easily take the DLM files with them after performing the lab because of the large size of the files. Secondly, the pre-service teachers need extensive training for creating DLM as they tend to treat them simply as digital papers with too much text, too less graphical bias and too few interactive elements. 


\title{
The Use of Digital Technologies in Physics and Mathematics Education by Partnership Pedagogy \\ Francisco Araújo ${ }^{1}$, Meirivâni Oliveira ${ }^{1}$ and Eloneid Nobre ${ }^{2}$ \\ ${ }^{1}$ Secretariat of Education of Ceara, Brazil, ${ }^{2}$ Federal University of Ceara
}

\begin{abstract}
In recent years it comes significant changes in the world, especially in relation to advances in Information and Communication Technologies (ICT). However, these changes are still poorly perceived in Brazilian public schools. Currently the students are increasingly dealing with the advances in ICT, especially in their mobile phones, having a increasing contact with new technologies. We realize that the traditional way of teaching can lead to discouragement, lack of interest, increasing problems such as avoidance, indiscipline and failure. Contrasting to the growing abilities of the students, there is still some resistance from some teachers, to insertion of digital technologies in their classes.

Following the Presnky Partnership Pedagogy and using low cost materials, this study aimed to provide exchange of information between teachers and students, through the use of Vernier Video Physics applications and the Vernier Graphical Analysis, in the study of the projectiles launch.

This work aims to encourage secondary school teachers to use multimedia technology resources in their classrooms. The project was developed in a public secondary school, located in Fortaleza, Ceará, Brazil, with the participation of teachers of physics and mathematics and high school students. Rockets were built with matchsticks and their releases were filmed. The students teach the teachers how to handle the applications, to make a video analysis of the launch of rockets. Analyzing the questionnaire applied for teachers, we found that $60 \%$ of them had never used any kind of applets in the classroom, and half of them, had difficulties in using this kind of technology in the classroom. Despite of their difficulties, all of them said that workshops using low cost materials combined with new technologies, may facilitate not only the learning, of Physics but also the mathematical functions needed to study the launching of projectiles, making the classes more dynamic and interesting, besides being an excellent alternative for schools that do not have appropriate science laboratories. Teachers also said they did not feel diminished in learning with their students and that this exchange of knowledge helps to improve the processes of teaching and learning because the students feel themselves part of the process.
\end{abstract}

\section{References}

GIRAFFA, L. M. M. Jornada nas Escol@s: A nova geração de professores e alunos. Revista Tecnologias, Sociedade e Conhecimento, (Schools Trek: The new generation of teachers and students. Magazine Technology, Society and Knowledge) Campinas, v.1, n.1, p. 100-118. Available in: http://www.nied.unicamp.br/ojs/ index.php/tsc/article/view/112/100. Last accessed on May 2, 2015.

PRENSKY, M. Teaching Digital Natives: partnering for real learning. California: Corwin, 2010. 


\section{Collection of Solved Problems and Collection of Experiments in Physics: Worthwhile Connection of Two Online Learning Sources Zdeňka Koupilová and Petr Kácovský \\ Charles University in Prague, Czech Republic}

\section{Introduction and Theory}

To solve quantitative physics problems is a key ability which students should reach during their physics education. But there is usually lack of time to solve enough problems during lessons to develop this competency enough; moreover, there is a very limited amount of suitable materials for home study. For this reason we started to develop a collection of fully solved problems nine years ago. The collection is specially designed to encourage students in active approach to problem solving, e.g. to solve at least some parts of a problem independently. Whereas both the database and the web interface turned out to be useful for the purposes of the collection of solved problems, the idea to develop its "sibling" - a collection of physical experiments - emerged four years ago. This collection aims primarily at physics teachers.

\section{Concept and Implementation}

All information and texts of problems and experiments are stored in MySQL database except for images and video. Texts of problems' sections are written in an extended version of XHTML language using several special tags for formatting the text and inserting other objects (like equation, images, links to another problem...). Formulas are written in LaTeX format and displayed by MathJax. Video clips are embedded using YouTube player.

The solution of each task is divided into sections hidden under ribbons containing only section title and the content is shown after user clicks on it. This implementation ensures that the reader does not see the whole solution and by various hints is motivated to solve the problem actively. The collection of experiments also uses the division of text into sections to keep the experiment description well-organized, but all sections are by default visible for the reader. The descriptions of experiments are supported by photo and video documentation as well as technical and methodical notes facilitating their performance.

\section{Objectives and Assessment}

Nowadays the database contains about 750 tasks in physics and 350 tasks in mathematics in Czech, more than one hundred tasks in Polish and more than 120 tasks in English. All tasks have structured solutions.

Besides this there are about 80 experiments in the collection of experiments. All of them provide detailed description supported by photos, short video clips and methodical tips for teachers. What is important, selected problems and experiments that deal with the same topic are interconnected to enable teachers to use them together.

We have very positive responses from user not only from Czech Republic. The collections together have more than 1500 unique visits per day (except for weekend and school holidays).

\section{References}

The collections are available at websites:

http://www.physicstasks.eu/

http://reseneulohy.cz/

http://fyzikalnipokusy.cz/ 


\author{
Students of the 21st century Learning Science: \\ Awakening future scientists through multimedia activities. \\ Márcio Nasser Medina ${ }^{1}$, Marcia Begalli ${ }^{2}$, Bianca Dantas Durães ${ }^{2}$, Yara Coutinho ${ }^{3}$ and Jose Seixas ${ }^{3}$ \\ ${ }^{1}$ Colégio Pedro II - Campus Niterói, Brazil, ${ }^{2}$ Universidade do Estado do Rio de Janeiro, Brazil, \\ ${ }^{3}$ Universidade Federal do Rio de Janeiro, Brazil
}

\title{
Introduction and Theory
}

This work presents an experience developed with students between 15 and 19 years old at a public school placed in the outskirts of Rio de Janeiro City. Through mutual collaboration between CERN, University and school, high school students discuss real experiments results of High Energy Particles Physics (HEPP) in virtual meetings with students around the world. The different multimedia platforms and social networks allow the students to work together, through the available resources on the world web, developing a network of knowledge and collective learning.

\section{Concept and Implementation}

"Hands-on Cern" is an activity developed in order to disseminate the latest discoveries made at CERN to society and also to stimulate new researchers. Our high school students analyze the events of the ATLAS and CMS experiments on the basis of real data in order to search for the Higgs boson which is blended with a large number of samples of $\mathrm{W}$ and $\mathrm{Z}$ events as if it were a treasure Hunt.

The masterclass enables the students to perform measurements on real data from experiments of physics particles using softwares as Hypatia and Minerva. First, they practice particle identification by exploring the characteristic signals left by particles in various detector elements where electrons, muons, photons and jets are recognizable. They go on selecting and categorizing events, and then they proceed with measurements. Typically, two students analyze 50-100 events before joining peers to combine and discuss data with the tutors at their local institution. Then they join students from several different locations to combine and discuss all the data in a video conference from CERN.

\section{Objectives and Assessment}

The IPPOG Masterclass - "Hands on CERN" demonstrated a new and innovative way of introducing the physics frontline in the classrooms. FOR years we HAVE SEEN that while the students are learning classical physics during their high school course, the Masterclass activity allows them to go ahead, dive deep into their own curiosities, try. Students participating in those activities progress much more in their studies when compared to the others. The internet proved to be an interesting and very useful research tool. Students enjoy very much the videos from YouTube, CERNTV, also from BBC, as well as those found on the masterclass website. 


\author{
Students of the 21st Century Learning Science: \\ Learning Physics Through Robot Design. \\ Márcio Nasser Medina ${ }^{1}$ and Márcia Sadok ${ }^{2}$ \\ ${ }^{1}$ Colégio Pedro II - Campus Niterói, Brazil, ${ }^{2}$ UniLaSalle - Campus Niterói, Brazil
}

\title{
Introduction and Theory
}

This project is a collaboration between the University La Salle (Unilasalle) and the Colégio Pedro II - Campus Niterói". The Unilasalle has a course in Information System (IS) where projects are developed that combine robotics, programming and technology, including the Lego MindStorm, Firefox OS and Arduino.

One of the educational aspects that sustain our research is constructivism and Seymour Papert, who proposes to provide the necessary tools so that students can discover and explore knowledge, so, the computers. According to this proposal, the learning process occurs through the realization of a concrete action that results in a tangible product that has a personal meaning for the learner. Consequently we learn best by doing.

\section{Concept and Implementation}

The group of high school students, will be called "apprentices", did not have any previous experience with robotics, nor with programming. Only a few had ever played with Lego, but only knew the MindStorm through the website. However, the graduation students will be called "veterans" as develop their projects for over 3 periods.

The selection of the participating students was performed using the following criteria: Academic achievement in the field of Physics and Mathematics and second individual performance in school contests of Astronomy, Physics, Mathematics and Robotics.

The Robotics Olympiad (OBR) is one of Brazil's scientific contest supported by CNPq that is used the theme of robotics. In the last OBR, which occurred in 2014, 9 of the 12 students obtained silver and bronze medals in the "theoretical" although they never had contact with robots or with any programming language.

\section{Objectives and Assessment}

This work presents an experience developed with students between 15 and 19 years old at a public school in a peripheral village of the city of Rio de Janeiro. Through mutual collaboration between UniLaSalle and school, high school students can construct and design LEGO robots testing their knowledge on physics. The different multimedia platforms and social networks allow the students to work together, through the available resources on the world web, developing a network of knowledge and collective learning. 
Poster Session

Room P (F 104 / Fakultätensitzungszimmer)

Contribution P22

Wed, 9 Sep, 16:30-18:30

\author{
Didactic Use of Teaching Energetics for High School Students \\ István Gärtner \\ ELTE University - Budapest, Hungary
}

\begin{abstract}
Teaching energetics in high school on secondary level is an important but difficult task. Partly the topic itself is closely related to other discipline too. The other reason is that the fact that the amount of energy required for daily living is significantly more than what we can produce with our muscle power is imperceptible and therefore hardly intelligible for the students. My poster presentation will contain the short description of my topic, a few calculation problems, which have surprising and thought-provoking results and some photos about the power generation system and about the buildings.

First I outline the changes of the energy consumption of two high schools in Budapest. (One is Fasori Gimnázium, which school is classified in this year as a historic site, and the other one is my working place: Árpád Gimnázium - Nagyszombat u. 19. , H-1034 Budapest)

Using the data and compared them to the past few years I will give a short calculation for the energy consumption per capita and per student groups in both institutions.

At my working place the same energy generating equipment has been working from several years but in Fasori Gimnázium most of the electrical power has been produced by special photovoltaic system for about half a year. (Before this the electric generating system were the same in both schools.) In addition to the comparison it allows the consideration of energy use in Fasori Gimnázium as I will show it with another short calculation sample task.

Secondly on my poster presentation the interpretation of data will be achieved through real examples. Using the data of the solar cells which were installed on the building of Fasori Gimnázium I determine the maximum available power of this system. In connection with this I present an everyday-life example which can be also known by students too that what size of muscle power create the same energy per unit time.

I would like to draw the attention how important, in addition to the traditional teaching curriculum, to interpret the knowledge we are facing everyday correctly. I hope the problems and their solving can help in it with this poster.
\end{abstract}




\title{
Experiences With the Use of Interactive Program Stellarium in the Educational Process \\ Mária Csatáryová and Ján Brajerčík \\ University of Prešov, Slovakia
}

\begin{abstract}
The authors of the article discuss possible incorporation of astronomical topics in the educational process in physics at secondary school. The method of virtualization as one of the innovative methods of teaching process is used. We designed the tasks on selected physical topic Newton's law of gravity, dynamics of particle, emission and absorption spectra - using virtual interactive Stellarium. Individual tasks were designed based on historical observations and are supported by investigative character. For example, the tasks include determination of the mass of Mars, determination of the speed of light and the Hubble Law of the universe expansion. To review the effectiveness of teaching process via virtual observations, two classes of upper secondary education were selected in the pilot project. Teaching process in one class was led by traditional way and in the second class teaching process was supported by the use of interactive Stellarium. The results of the knowledge test have shown that teaching process with incorporation of astronomical topics using virtual observation was effective. The results also imply that students taught with the support of interactive program Stellarium, were in the process of teaching more active and showed better results fixation.
\end{abstract}




\begin{tabular}{|c|c|}
\hline Al-Shamali, Farook & 77 \\
\hline Anada, Yuichi & 126 \\
\hline Andres, Thomas & 127 \\
\hline Appel, Julia & 127 \\
\hline Araújo, Francisco & 133 \\
\hline Arieli, Rami & 103 \\
\hline Barroso, Marta Feijó & 79 \\
\hline Begalli, Marcia & 135 \\
\hline Beishuizen, Jos & 110 \\
\hline Bisalla, Ibrahim Iliya & 80 \\
\hline Bischke, Benjamin & 43 \\
\hline Blankert, Piet & 35,99 \\
\hline Bobroff, Julien & 86 \\
\hline Bonanno, Assunta & 78 \\
\hline Bouquet, Frédéric & 86 \\
\hline Bozzo, Giacomo & $25,78,96$ \\
\hline Braithwaite, Nicholas & 33 \\
\hline Brajerčík, Ján & 138 \\
\hline Bresges, André & $67,69,70$ \\
\hline Brodeur, Marcus & 33 \\
\hline Bronner, Patrick & 87 \\
\hline Bungum, Berit & 20 \\
\hline Büsch, Leonard & 120 \\
\hline van Buuren, Onne & 109 \\
\hline Carreras, Carmen & 31 \\
\hline Chevrier, Joël & 64 \\
\hline Christensen, Warren & 129 \\
\hline Christian, Wolfgang & 85 \\
\hline Coutinho, Yara & 135 \\
\hline Csatáryová, Mária & 138 \\
\hline Csernovszky, Zoltán & 95 \\
\hline Da Silva, Rodrigo L. & 40 \\
\hline Da Silva, Samir L. & 40 \\
\hline De Ambrosis, Anna & 47,94 \\
\hline Dębowska, Ewa & 38 \\
\hline Dede, Chris & 81 \\
\hline Deibl, Ines & 68 \\
\hline De Luca, Claudio & 96 \\
\hline van Den Berg, Ed & 110 \\
\hline Dershimer, R. Charles & 128 \\
\hline Dormido, Sebastián & $31,52,53$ \\
\hline Dubson, Michael A. & 13,67 \\
\hline Dunlap, Justin C. & 129 \\
\hline Durães, Bianca Dantas & 135 \\
\hline Dür, Wolfgang & 46 \\
\hline Ebini, Raiya & 49,113 \\
\hline Eisenkraft, Arthur & 81 \\
\hline Ellermeijer, Ton & 109,110 \\
\hline Erkelenz, Jan & 121 \\
\hline Ertl, Bernhard & 72 \\
\hline Esquembre, Francisco & 52,73 \\
\hline
\end{tabular}

Fagundes, Adriano Luiz $\quad 79$

Finkenberg, Frank 132

Fischer, Christian 81, 128

Fishman, Barry 81

Franz, Torsten 88

Frumin, Kim 81

Galan, Daniel 52

García-Salcedo, Ricardo 116

Gärtner, István 137

Genz, Florian 69

Giampà, Salvatore 93

Girwidz, Raimund 17, 30, 32,

125

González, Manuel Á. $\quad 62$

González, Miguel Ángel 62

Greczyło, Tomasz 37, 38

Gregorcic, Bor 23, 24

Grimalt-Alvaro, Carme $\quad 25$

Gröber, Sebastian 65, 104

Guaitolini, Judismar T. Junior 40

Hackenberger, Christian 14

Hamacher, John 121

Haruna, Isiaku 80

Heck, André $\quad 109$

Heinke, Heidrun $\quad 120,121$

Hein, Ulla 127

Hemmer, Sabine $\quad 117$

Heradio, Ruben 52

Herbst, Markus 39

Heusler, Stefan 21, 46, 89

Hirth, Michael 43, 61

Horváth, Ákos 95

Humphreys, Carl 84

Jarosievitz, Beáta 92

Jeanjacquot, Philippe 63

Jende, Konrad 71, 123

Jia, Yueming 81

van Joolingen, Wouter $\quad 15,118,119$

Jutant, Camille 86

Kácovský, Petr 134

Keller, Oliver 70, 123

Klein, Pascal 65, 104

Koenig, Kathleen 105

Kohnle, Antje 83, 84

Kolb, Ulrich 33

Kook, Janna Fuccillo $\quad 81$

Koupilová, Zdeňka $\quad 57,134$

Krijtenburg, Kim 118, 119

Kuhn, Jochen

$42,43,59,60$,

$61,65,104$

Kuik, Gerrit

35,99

Kutschera, Ellynne 129 


\begin{tabular}{|c|c|c|c|}
\hline Landua, Rolf & 71 & Sadok, Márcia & 136 \\
\hline Langley, Dorothy & 103 & Sáenz, Jacobo & 31,53 \\
\hline Laumann, Daniel & 21 & Sánchez Guzmán, Daniel & 111,116 \\
\hline Lawrence, Ian & 23,37 & Sánchez, Jose & 31 \\
\hline Lawrenz, Frances & 81 & Sánchez, Juan Pedro & 31 \\
\hline Laws, Priscilla W. & 105 & Sapia, Peppino & 78,96 \\
\hline Leal, Fábio F. & 40 & Scerbo, Francesco & 93 \\
\hline Leccia, Silvio & 19 & Schieck, Jochen & 12 \\
\hline Levy, Abigail Jurist & 81 & Schmeling, Sascha & 123 \\
\hline Liang, Xi Xia & 130 & Schwingel, Alexander & 127 \\
\hline Lindlahr, William & 27 & Scordo, Elena & 93 \\
\hline López Simó, Víctor & $17,18,25$ & Seixas, Jose & 135 \\
\hline López Tavares, Diana B. & 116 & Şen, Ahmet İlhan & 55 \\
\hline Lowe, David & 16,29 & Silberhorn, Christine & 87 \\
\hline Lück, Stephan & 131 & da Silva, Tatiana & 79 \\
\hline \multirow[t]{2}{*}{ Lukowicz, Paul } & 43 & Sirigu, Mauro & 122 \\
\hline & & Smeets, Pieter & 107,108 \\
\hline Malgieri, Massimiliano & 47,94 & Stanislav, Vladimír & 57 \\
\hline McCoy, Ayana & 81 & Strahl, Alexander & 39,68 \\
\hline Medina, Márcio Nasser & 135,136 & Strunz, Andreas & 87 \\
\hline Meng, Qinyun & 41 & Struzyna, Sarah & 54 \\
\hline Meyn, Jan-Peter & 87 & & \\
\hline Michelini, Marisa & 26,97 & Teese, Robert B. & 105 \\
\hline Minocha, Shailey & 33 & Testa, Italo & 19 \\
\hline Molz, Alexander & 42 & Theyßen, Heike & 54 \\
\hline Montalbano, Vera & 76,122 & Thoms, Lars-Jochen & $29,30,125$ \\
\hline Morel-Deville, Françoise & 63 & Titus, Aaron & 73 \\
\hline Moretto, Sandra & 117 & Tornow, Sabine & 48 \\
\hline Moser, Stephanie & 68 & de la Torre, Luis & $31,52,53$ \\
\hline Mulder, Jan & 35,99 & Tran, Trinh Ba & 110 \\
\hline Müller, Andreas & $\begin{array}{l}42,59,60,61 \\
65\end{array}$ & Trefzger, Thomas & 132 \\
\hline Müller, Rainer & 83 & Üstün, Ulaş & 49,113 \\
\hline \multirow[t]{2}{*}{ Mylott, Elliot } & 54,129 & & \\
\hline & & Vercellati, Stefano & 26,97 \\
\hline Nagel, Andrea Lisa & 125 & Vero, Laura & 93 \\
\hline Nobre, Eloneid & 124,133 & Viana, Emilson R. & 40 \\
\hline \multirow[t]{2}{*}{ Nolan, Sam J. } & 34 & Vícha, Vladimír & 57 \\
\hline & & Vieyra, Rebecca & 69 \\
\hline Oktay, Özlem & 55 & & \\
\hline Oliveira, Meirivâni & 133 & Wang, Yun & 41 \\
\hline \multirow[t]{2}{*}{ Onorato, Pasquale } & 47,94 & Wee, Loo Kang Lawrence & 102 \\
\hline & & Wendt, Klaus & 27 \\
\hline Paetkau, Mark & 84 & Weppner, Jens & 43 \\
\hline Pantano, Ornella & 117 & Westkamp, Nadine & 70 \\
\hline Pereira, Caniggia & 124 & Widenhorn, Ralf & 54,129 \\
\hline Pol, Henk & $107,118,119$ & Woithe, Julia & 123 \\
\hline Poxrucker, Andreas & 43 & & \\
\hline Puddu, Emanuella & 19 & Yuste, Manuel & 31 \\
\hline Richtberg, Stefan & 32 & Žilavý, Peter & 57 \\
\hline van Rossum, Aernout & 119 & Zollman, Dean & 49,113 \\
\hline Roth, Tobias & 127 & Zumbach, Jörg & 68 \\
\hline
\end{tabular}


Notes 
Notes 
Notes 
Notes 


\section{Your Way to the Reception Desk (R) and the Seminar Rooms (A, B \& C)}

1. Face the LMU main building (Hauptgebäude).

2. Take the first door on the right in front of the main entrance.

3. Go upstairs to the intermediate floor (Zwischengeschoss).

4. Find the reception desk on the left.

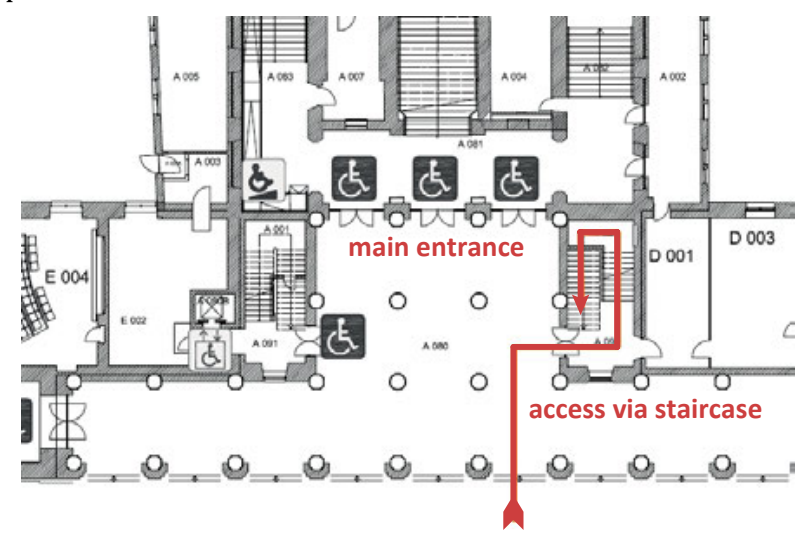

Geschwister-Scholl-Platz 1

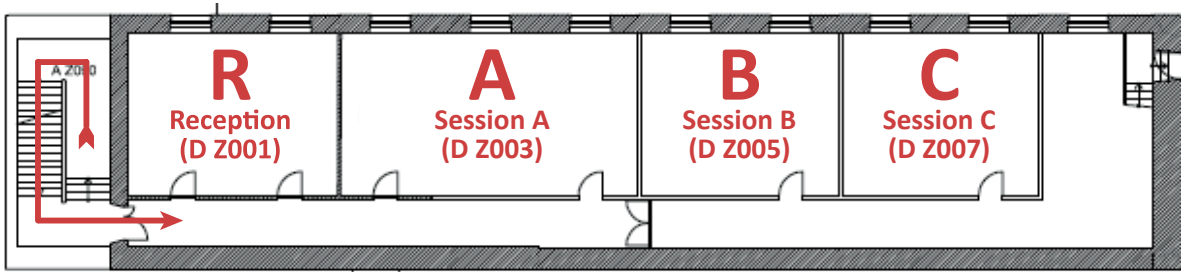

Your Way to the Lecture Hall (H)

Plenary lectures will be held in the lecture hall D 209 in the second floor above the reception desk and the seminar rooms.

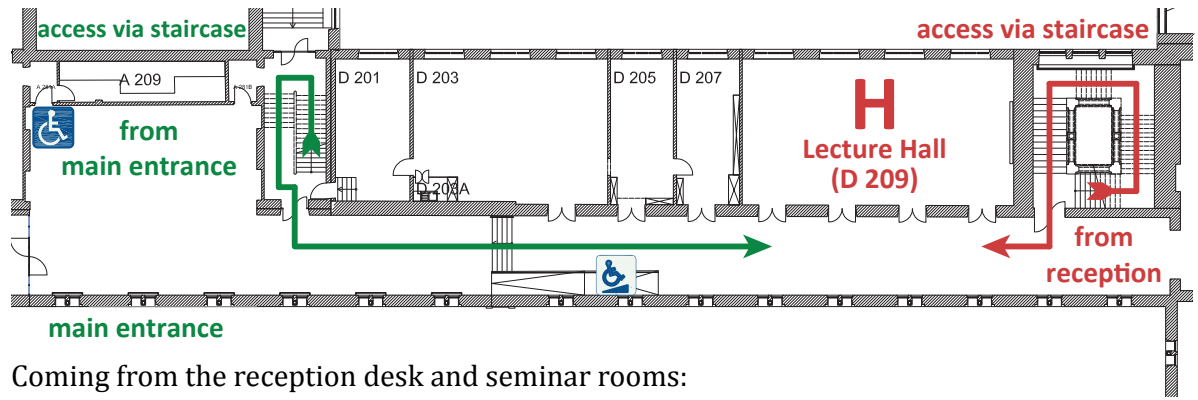

1. Go upstairs to the second floor.

2. Leave the staircase, turn right.

3. Find the lecture hall on the right.

Coming from the main entrance:

1. Face the LMU main building (Hauptgebäude).

2. Take the first door on the right in front of the main entrance.

3. Go upstairs to the second floor, leave the staircase, turn left, go straight forward.

4. Find the lecture hall on the left. 


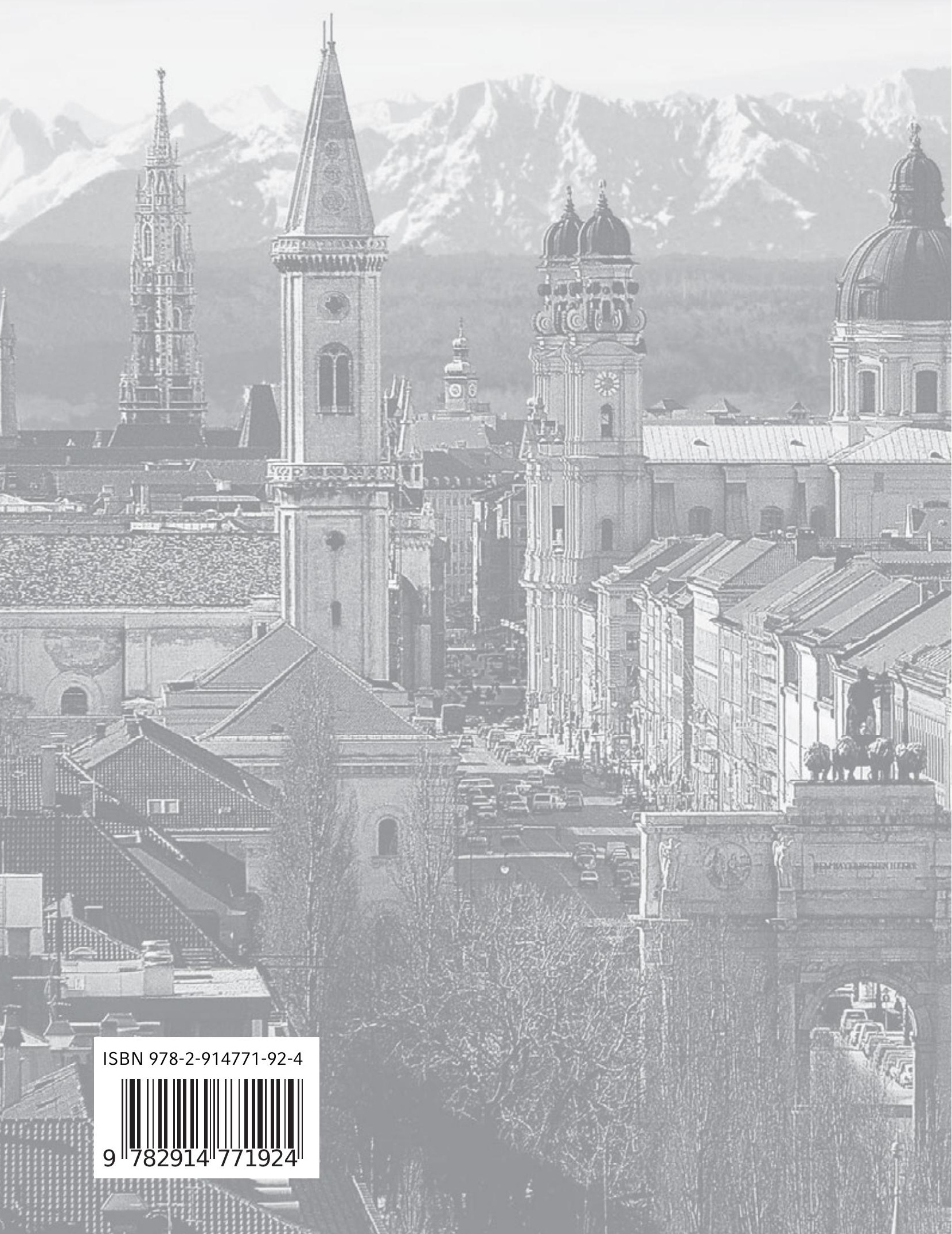

